Metals and Ceramics Division

CERAMIC TECHNOLOGY PROJECT DATABASE:

SEPTEMBER 1990 SUMMARY REPORT

B. L. P. Keyes

June 1992

NOTICE: This document contains information of a preliminary nature. It is subject to revision or correction and therefore does not represent a final report.

Prspared for the

U.S. Department of Energy

Assistant Secretary for Conservation And Renewable Energy

Office of Transportation Technulogies

EE 5101000

Prepared by the

OAK RIDGE NATIONAL LABORATORY

Oak Ridge, Tennessee 37831-6285

managed by

MARTIN MARIETTA ENERGY SYSTEMS, INC.

for the

U. S. DEPARTMENT OF ENERGY

under contract DE-AC05-840R21400 


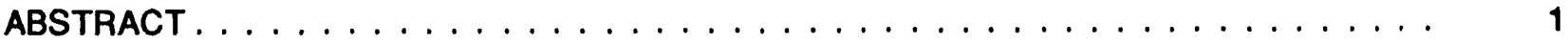

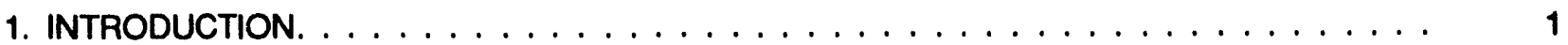

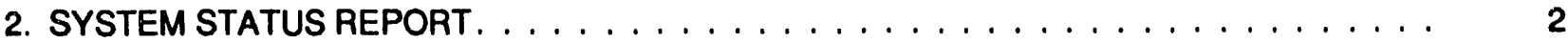

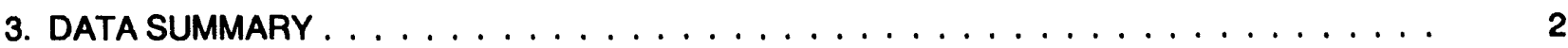

4. SYSTEM ACCESS $\ldots \ldots \ldots \ldots \ldots \ldots \ldots \ldots \ldots \ldots \ldots$

5. FUTURE PLANS $\ldots \ldots \ldots \ldots \ldots \ldots \ldots \ldots \ldots \ldots \ldots$

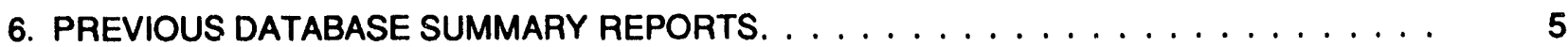

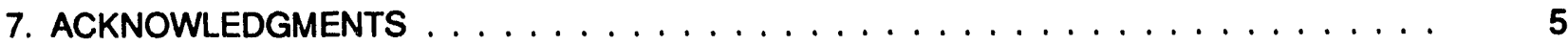

APPENDIX A: MATERIAL CHARACTERISTICS AND BACKGROUND

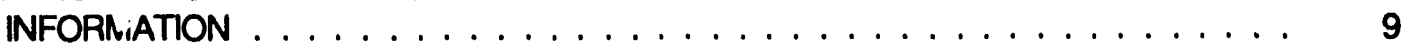

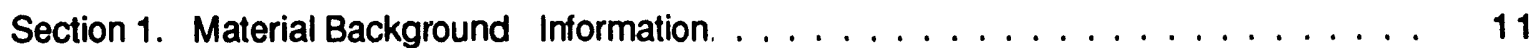

Section 2. Material Characteristics at Room Temperature . . . . . . . . . . . . . . . 13

Section 3. General Material Text Information. . . . . . . . . . . . . . . . . . . . 15

Section 4. Chemistry. . . . . . . . . . . . . . . . . . . . . . . . . . 19

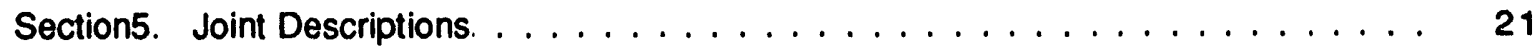

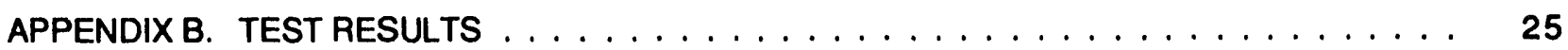

Section 1. Test Background Information . . . . . . . . . . . . . . 27

Section 2. Creep Data . . . . . . . . . . . . . . . . . . 31

Section 3. Fracture Toughness Data . . . . . . . . . . . . . . . . . 33

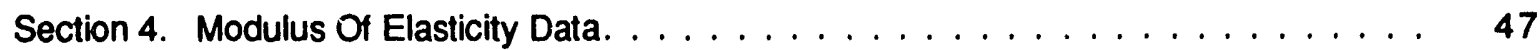

Section 5. Modulus of Rupture - 4-Point Bend Data . . . . . . . . . . . . . . 50

Section 6. Torsion Shear Strength . . . . . . . . . . . . . . . . . . . . 62

Section 7. Modulus of Rupture -4-Point Bend of Brazed Specimens . . . . . . . . . . . . 63

Section 8. Shear Strength of Brazed Specimens . . . . . . . . . . . . . . . . . . 69

Section 9. Torsion of Brazed Specimens . . . . . . . . . . . . . . . . . . . . . . 75

Section 10. Torsion Fatigue of Brazed Specimens. . . . . . . . . . . . . . . . 76 


\title{
CERAMIC TECHNOLOgY PROJECT DATABASE: SEPTEMBER 1990 SUMMARY REPORT*
}

\author{
B. L. P. Keyes
}

\begin{abstract}
Data generated within the Ceramic Technology Project (CTP) represent a very valuable resource for both research and industry. The CTP database was created to provide easy access to this information in electronic and hardcopy forms by using a computerized database and by issuing periodic hardcopy reports on the database contents. This report is the sixth in a series of semiannual database summaries and covers recent additions to the database, including joined brazed specimen test data.
\end{abstract}

\section{INTRODUCTION}

Data generation is an ongoing process in research. New materials are constantly developed, tested, modified, then retested, producing a vast quantity of information that should be archived and made available to other interested parties. The responsible researchers publish reports on their work, but these are not always easy to obtain, or even to find, several years after publication. Unless the work was well documented and archived, its usefulness will be limited to only a few individuals. Years later, the same work may be done again, with the same results, if no reference to the previous work exists. "Reinventing the wheel" is a waste of valuable resources. Such wastefulness must be avoided.

The CTP database was created to help avoid unnecessary testing redundancy by making available the work on material characterization and mechanical properties data generated within the CTP. Data are collected and stored in a timely manner using dBASE IV'm on an IBM PC/AT microcomputer. Users may access the data electronically by requesting information on specific materials or by requesting the entire database from the data base administrator. The data will be sent on floppy disks in the user's requested format. Direct access to the master files is limited to avoid accidental data contamination, or deletions, and to reduce the opportunity for exposure to computer viruses and other diseases. Hardcopy reports, like this one, are issued semiannually to inform the user community of the latest additions to the database and to give a system status update.

"Research sponsored by the U.S. Department of Energy, Assistant Secretary for Conservation and Renewable Energy, Office of Transportation Technologies, as part of the Ceramic Technology Project of the Materials Development Program, under contract DE-AC05-84OR21400 with Martin Marietta Energy Systems, Inc. 


\section{SYSTEM STATUS REPORT}

The database presently contains about 6,093 test results on over 360 different batches of ceramic materials. Approximately $47.8 \%$ of these are on zirconia-based ceramics, $7.1 \%$ are on silicon carbides, $21.8 \%$ are on silicon nitrides, $6.4 \%$ are on whisker-reinforced silicon nitrides, $15.3 \%$ are on alumina-based ceramics (including whisker-reinforced aluminas and mullites), and $1.6 \%$ are on other ceramics. Table 1 provides an overview of the data presently stored in the system in more detail.

Test data files on brazed-specimen torsion strength and torsion fatigue were added to the database during this summary period. Although the testing procedures for unjoined and joined specimens may be the same, additional information is needed to characterize the joint and all the materials used to create it. Often, dissimilar materials are brazed together, as in the case of the MS-PSZ and nodular castiron joints made by M. L. Santella at Oak Ridge National Laboratory (ORNL). To characterize such a joint requires information on all the materials involved, the actual joining process, and the test results. Detailed information on a given joint may be found in a separate file referenced by the joint code included in each joined-specimen test record. The JOINTEXT file is in text form and contains all the information about the brazing process and materials that could be found.

\section{DATA SUMMARY}

The data in this report cover 1 silicon carbide, 34 silicon nitrides, 10 whisker-reinforced silicon nitrides, 2 zirconia-toughened aluminas, 8 zirconias, and 34 joints. The data were taken from the CTP semiannual and bimonthly progress reports, contributions by K. C. Liu and M. L. Santella of ORNL, and a report prepared by GTE Laboratories, Analytical and Experimental Evaluation of Joining Silicon Nitride to Metal and Silicon Carbide to Metal for Advanced Heat Engine Applications, ORNL/SUb/87-SB047C/1, dated April 1990.

Data tables for this report are organized according to type of data: Appendix A contains available background information on the ceramics presented in this report; Appendix $B$ contains the experimental test data arranged in sections by property type. Some of the test data listed in Appendix B may not be represented by materials in Appendix $A$ but will be included in a later report when such information becomes available. It is the policy for this database to store the available information for future use, whether or not a complete set of information is available on a material. However, complete sets are preferred and sought as time and funding permit.

Some of the plots in this report compare properties of several materials by using bar charts. This was necessary because only one test temperature was used for many tests, thus providing no temperature range for an $X-Y$ plot. 
All Weibull analyses shown in this report were performed using the rank regression method, in which a least-squares fit to the data was used to estimate the modulus. Specifically, the data were sorted and the $X-Y$ coordinates, In [modulus of rupture (MOR) strength] and InIn [1/(1-F)], were calculated using Microsoft EXCEL ${ }^{\mathrm{TM}}$ (version 2.0) on an Apple Macintosh computer. The data were then transferred to Cricket Graph ${ }^{\mathrm{m}}$ (version 1.3.1), where they were plotted with the best-fit line, using Cricket Graph's built-in, curve-fitting routines.

Tracking down background information for a specific batch of a material is a time-consuming experience, which is often necessary to confirm the validity of the data. Keeping assigned batch numbers with the material as it goes through testing appears to be neglected in some cases. Some of the data in this reprort include the original batch codes assigned to the materials by the fabricators, but when no batch identifier is mentioned, a code is assigned by database personnel based upon the source (report code) and the company, laboratory, or individual who reported the data. In the case of materials with locally assigned batch codes, the possibility exists that the same material is also reported elsewhere in the database with the original fabricator's code.

\section{SYSTEM ACCESS}

Direct access to the master database is very limited to protect the integrity of the master files. Past experience has shown that major disasters often occur when too many people have direct access to unprotected files at the microcomputer level. Since most users would prefer to have the data in a familiar format, to subset, analyze, and rearrange to suit their needs, this method satisfies both situations; the master files are protected, and the user gets the data in a readily consumable form. While direct access is faster, the process of downloading across phone lines can be time consuming and hazardous to the integrity of the data being transmitted. This database was designed as a repository, not a full-function analytical tool.

Access to the data is attained by cailing the database administrator and requesting all files or just those pertaining to certain materials or test types. The CTP database is a microcomputer-based system of files organized in dBASE IVTM structure. The information requested by the user (in the user-designated format, including software and disk type) will then be downloaded from the master files, reformatted if necessary, then sent to the user. At this time, no plans are being considered for direct access from outside systems. Direct transfer is available by special arrangement but may be time consuming due to the sizes and numbers of files. No guarantee is given for the validity of data transmitted directly because of possible phone line problems. Until a computerized interface becomes available, the CTP Database User's Guide will be sent to all first-time users when the document becomes available. 
Several file formats, other than ABASE IVTM, are available for files downloaded for users. These formats have been categorized as either Apple Macintosh-compatible files (on 3.5-in. floppy disks) or IBM $\mathrm{PC}$-compatible files (on 3.5-in. floppy disks up to $1.44 \mathrm{MB}$ or 5.25 -in. floppy disks from $360 \mathrm{kB}$ to 1.44 MB). When requesting information from the database, users should indicate disk size, disk density, and file type.

Macintosh file types available are Microsoft EXCEL ${ }^{\mathrm{TM}}$, FOXBASE ${ }^{\mathrm{TM}}$, SYLK, and plain, printable ASCII. IBM file types are Lotus 1-2-3, Microsoft EXCELTM, DIF, SDF, SYLK, plain ASCII, delimited ASCII, dBASE IVTM, and dBASE III ${ }^{\mathrm{TM}}$. Other formats may be available by special arrangement.

With computer diseases becoming so rampant, users should be aware that precautions are taken to ensure that the disks they receive from the database are disease free. Only new disks are used for transmittals to avoid spreading any computer diseases that might be hiding. No recycled, reformatted disks are sent to users. Both the master system, a Northgate Elegance 425, and the Macintosh llcx are checked regularly for such illnesses; none have been found so far. Use of both computers is limited to one person who carefully screens incoming software to avoid contamination of either system. Information is not downloaded from public bulletin boards to either system. Both systems have virus detection software installed, and all efforts are made to ensure that both systems remain disease free. If users have problems with disks received from the database, they should inform database personnel immediately so that steps can be taken to correct the problems.

\section{FUTURE PLANS}

Plans are being made to write a computerized user interface using the dBASE IVTM programming language and to have the initial version completed some time in 1992. The interface will link the database's many files together, providing better access to information in the system. This interface will be available to all users of the database who request it.

The next hardcopy update is scheduled for draft completion in September 1992.

\section{PREVIOUS DATABASE SUMMARY REPORTS}

1. M. K. Booker, Ceramics Technology for Advanced Heat Engines Project Data Base: A Summary Report, ORNL/M-462, Martin Marietta Energy Systems, Inc., Oak Ridge Natl. Lab.

2. B. L. P. Booker, Ceramics Technology for Advanced Heat Engines Project Data Base: September 1988 Summary Report, ORNL/M-755, Martin Marietta Energy Systems, Inc., Oak Ridge Natl. Lab., March 1989. 
3. B. L. P. Keyes, Ceramics Technology for Advanced Heat Engines Project Data Base: March 1989 Summary Report, ORNL/M-1098, Martin Marietta Energy Systems Inc., Oak Ridge Natl. Lab., April 1990.

4. B. L. P. Keyes, Ceramics Technology for Advanced Heat Engines Project Database: September 1989 Summary Report,"ORNL/M-1286, Martin Marietta Energy Systems, Inc., Oak Ridge Natl. Lab., October 1990.

5. B. L. P. Keyes, Ceramics Technology Project Data Base: March 1990 Summary Report, Martin Marietta Energy Systems, Inc., Oak Ridge Natl. Lab., in publication.

\section{ACKNOWLEDGMENTS}

The author thanks Mike Santella and David Alexander (both from ORNL) for reviewing this document, Jeff Swab (U.S. Army Materials Technology Laboratory) for his contribution of the AMTL data, and the Metals and Ceramics Division Records Otfice for editing and preparing the final draft for publication. 


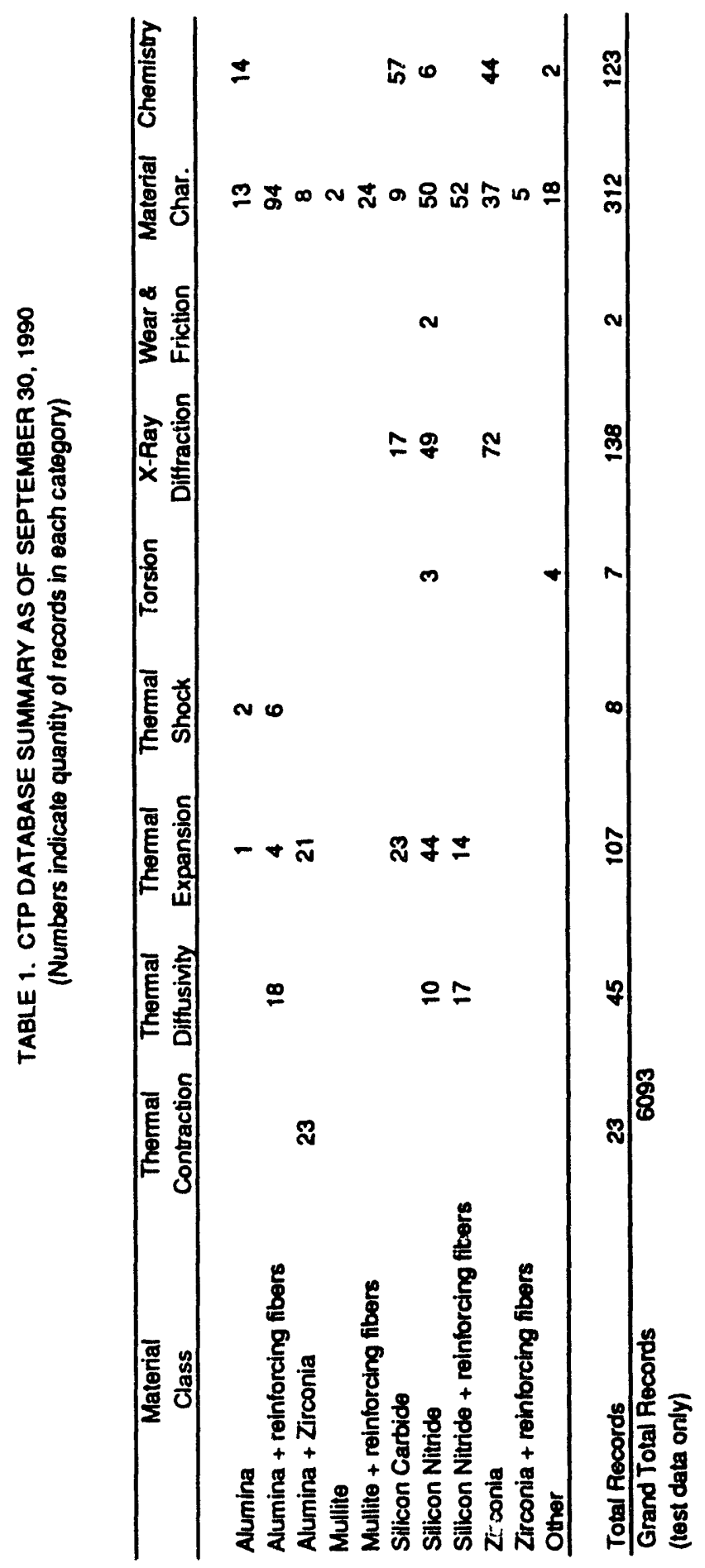

6 

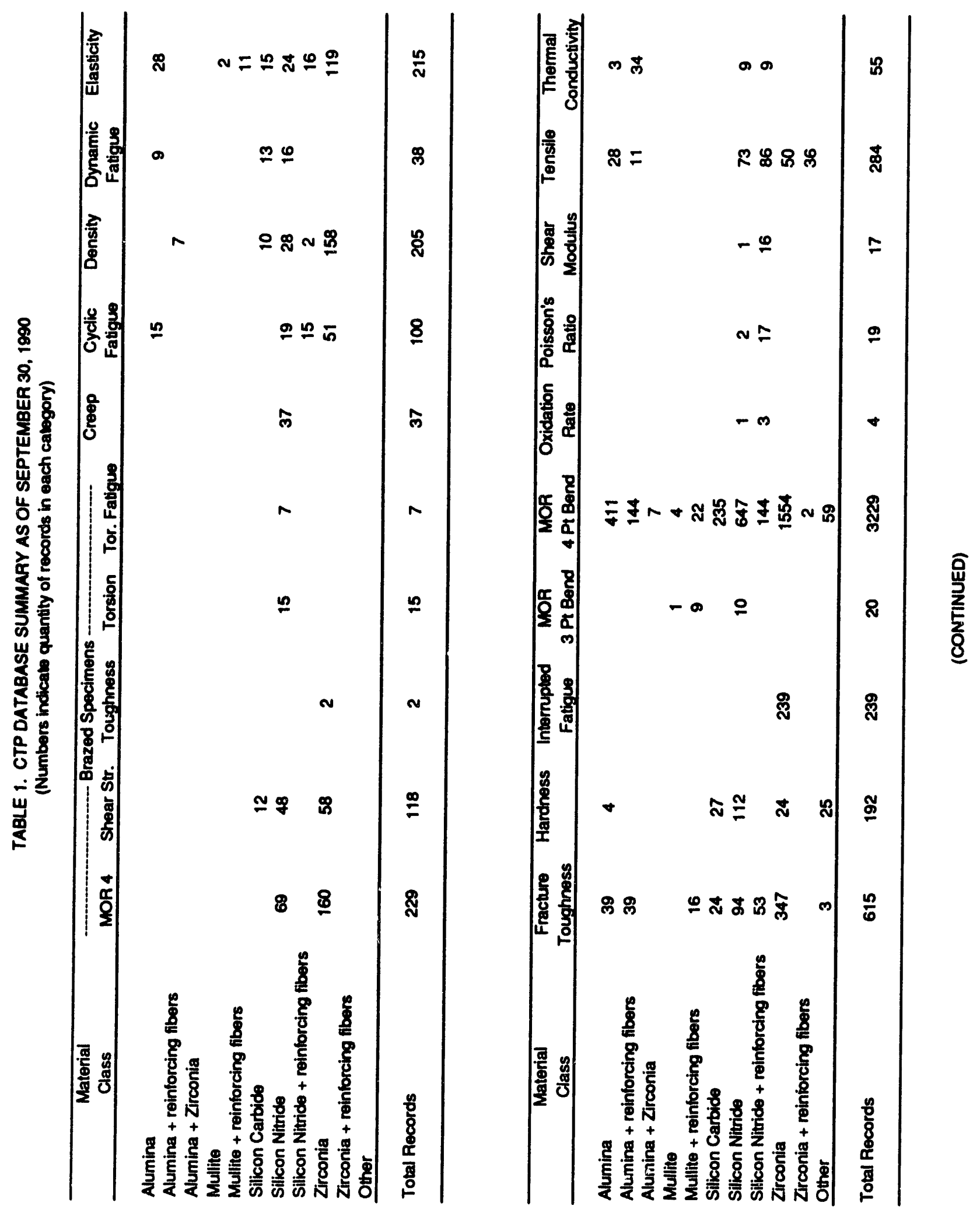


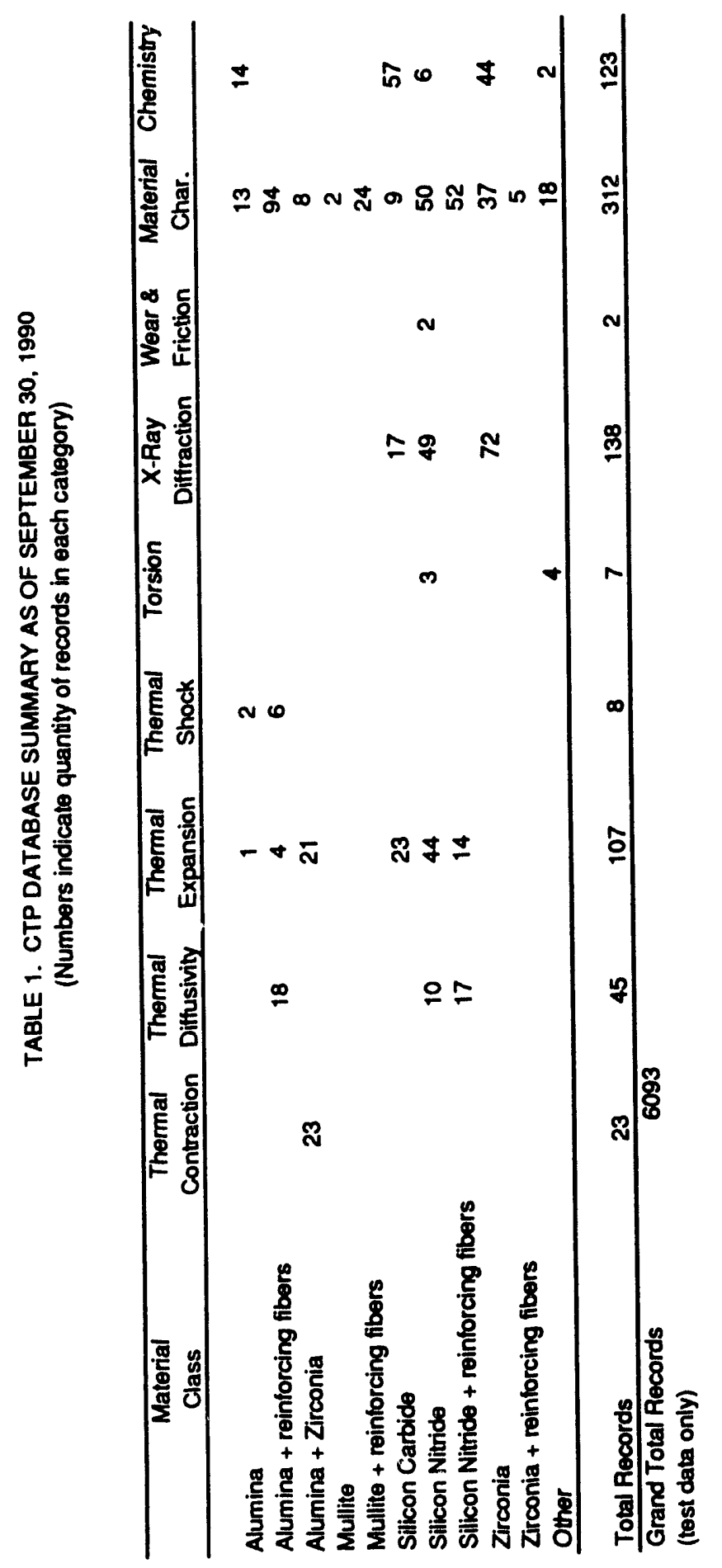


APPENDIX A. MATERIAL CHARACTERISTICS AND BACKGROUND INFORMATION 


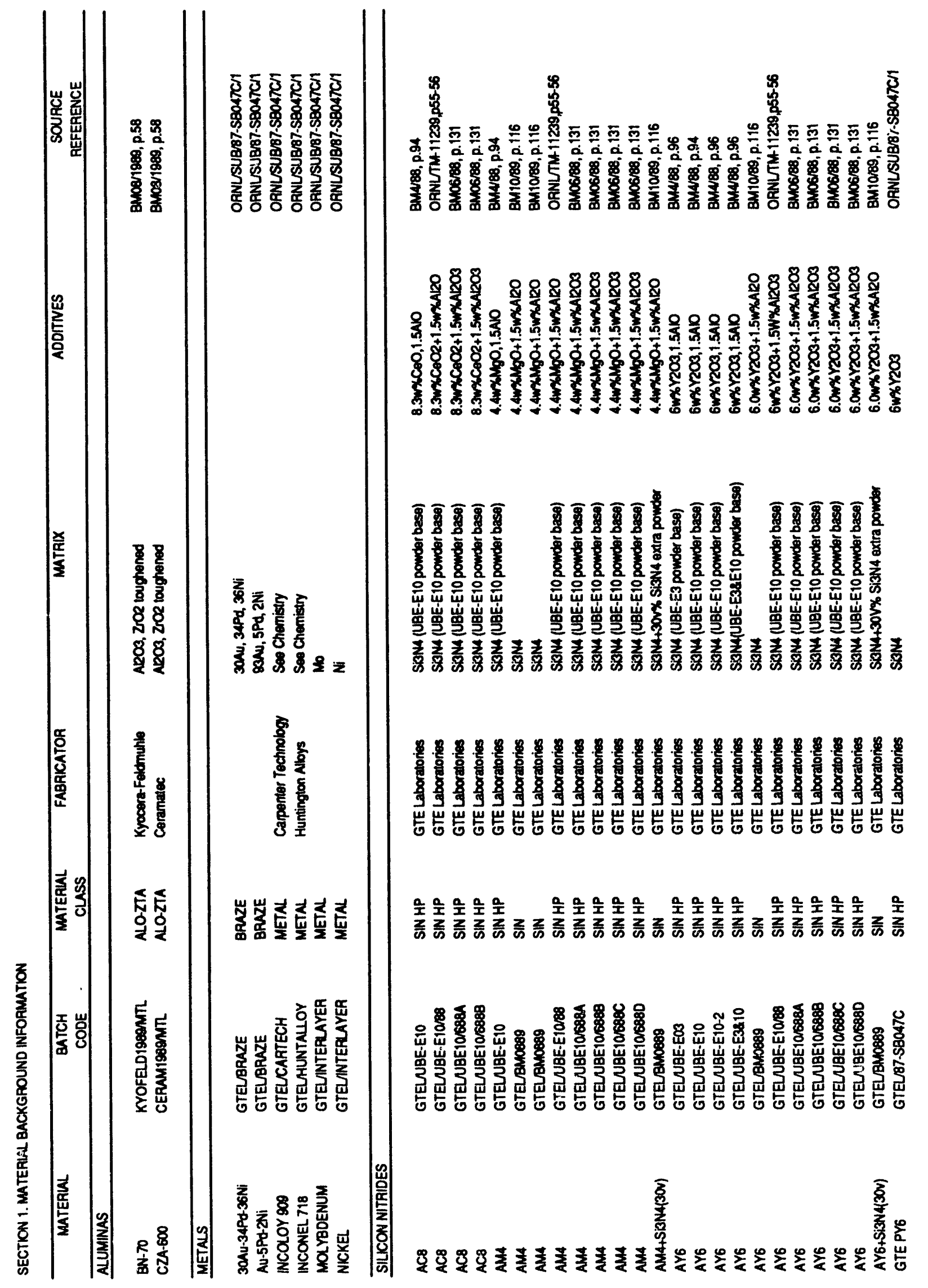




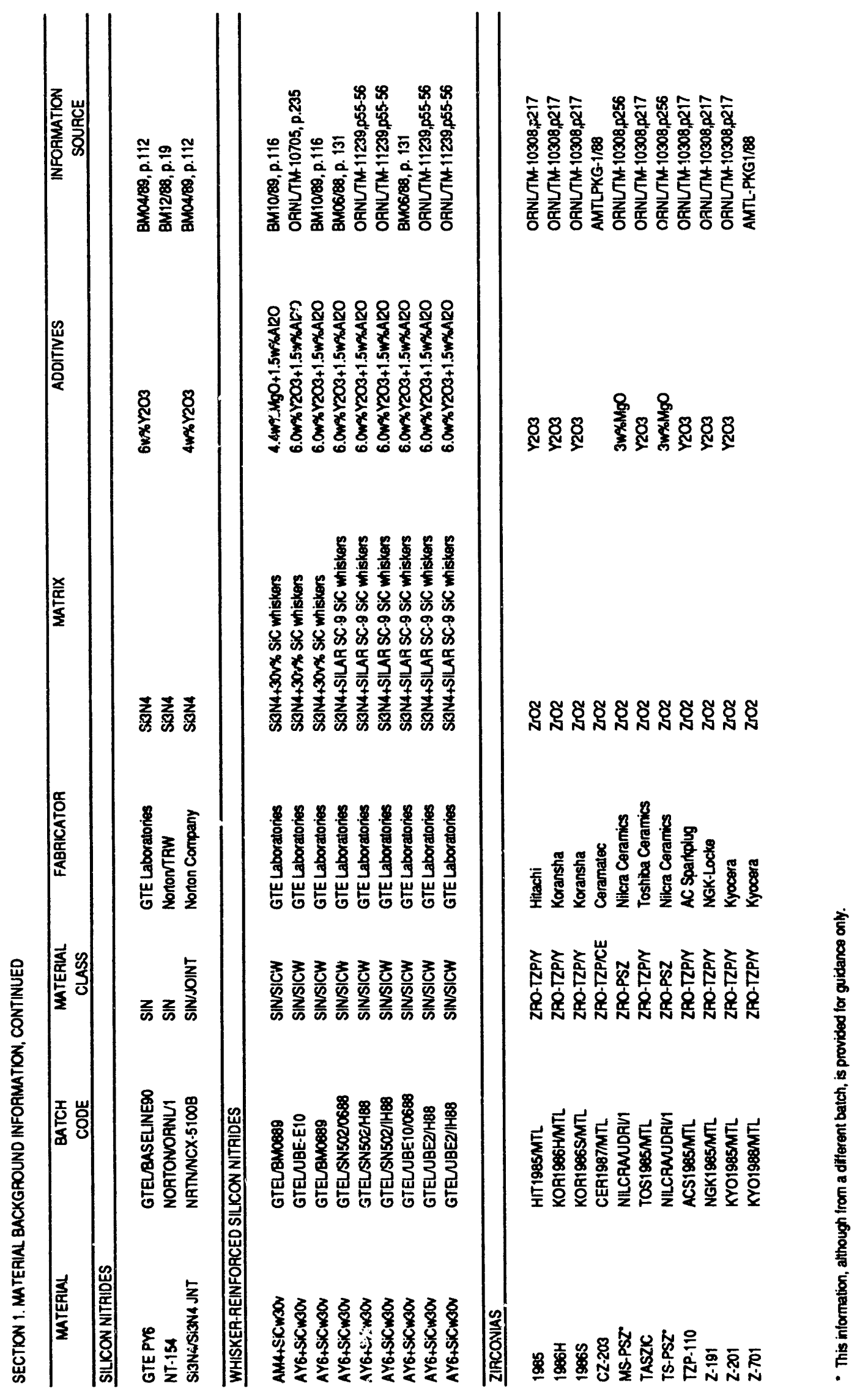




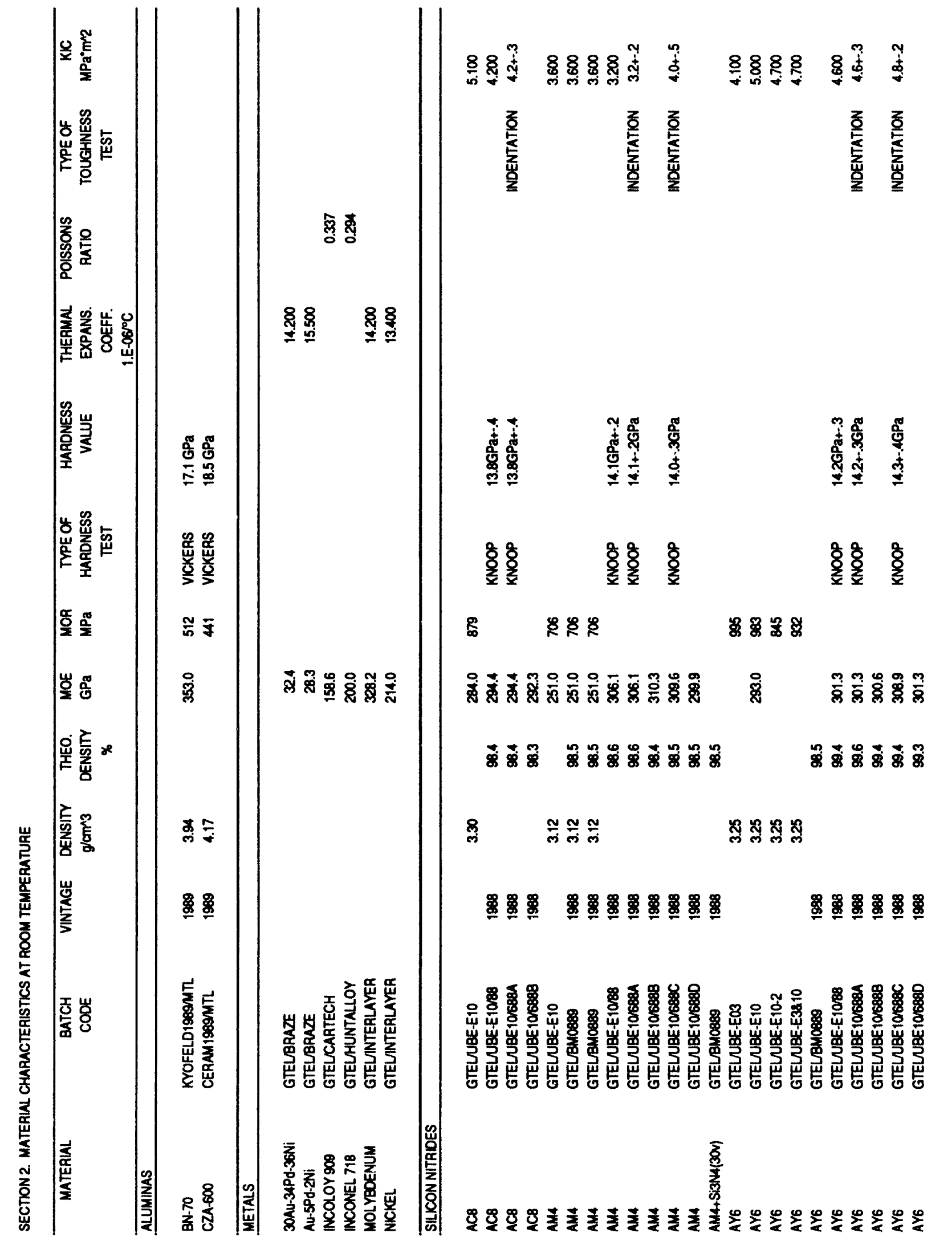




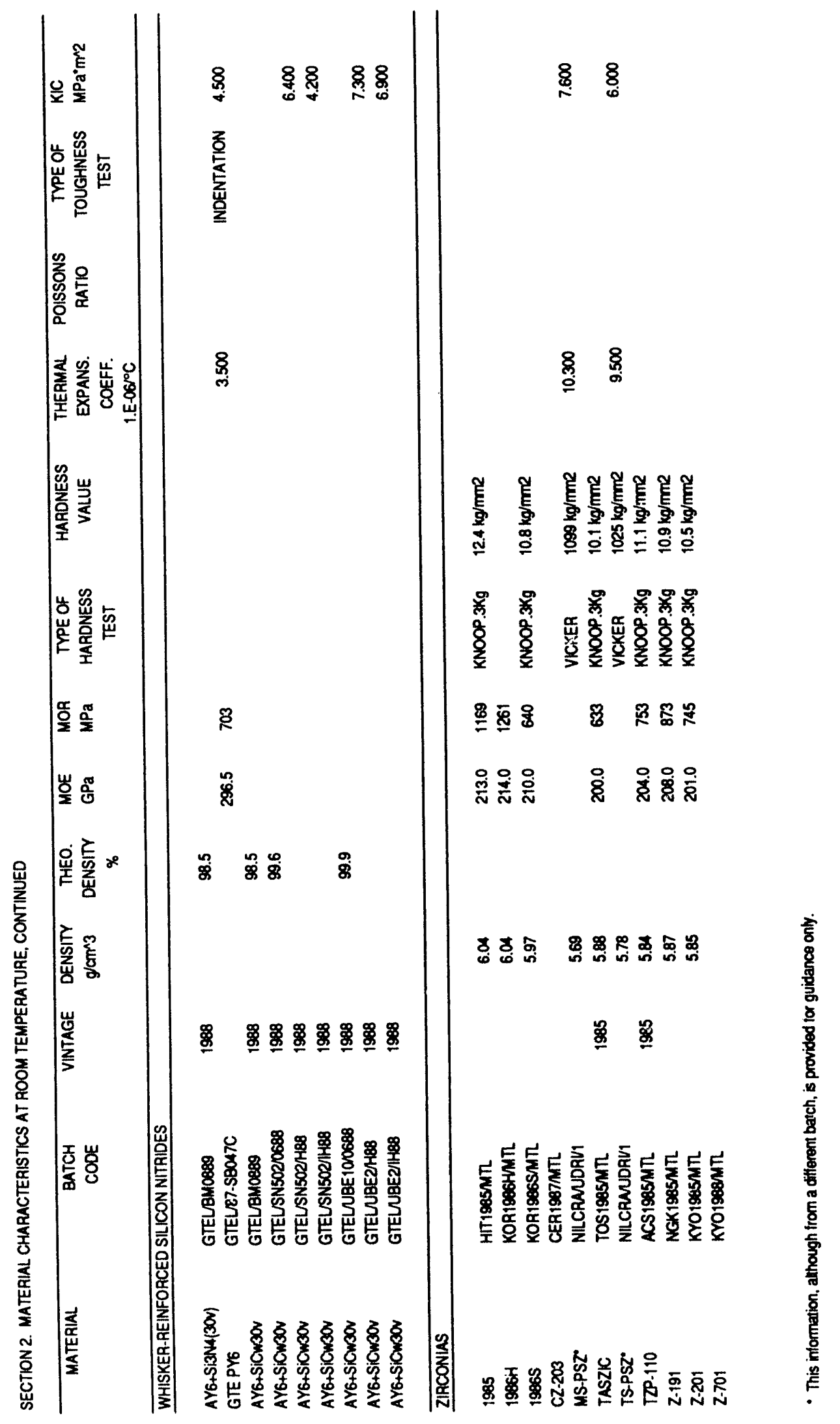




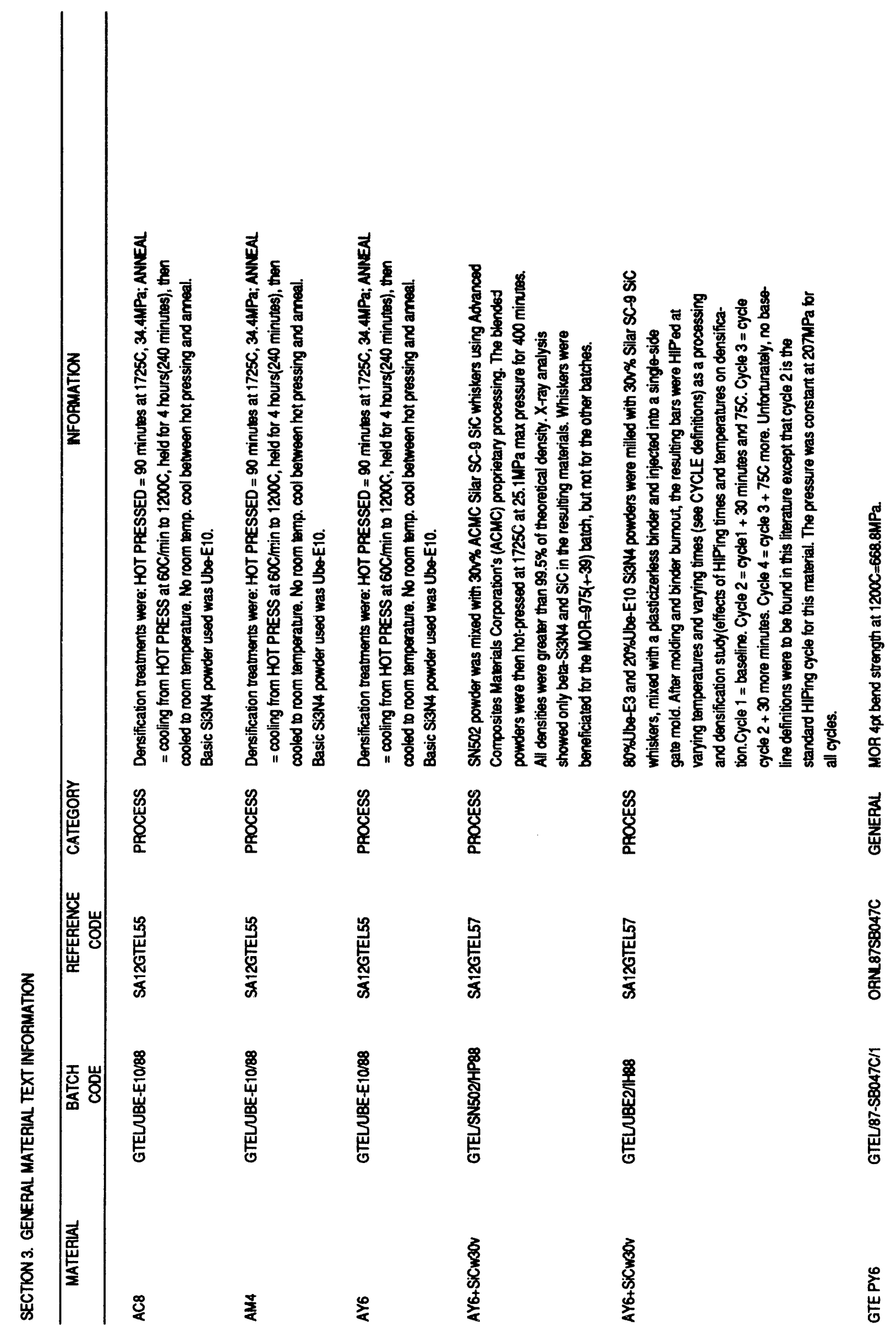




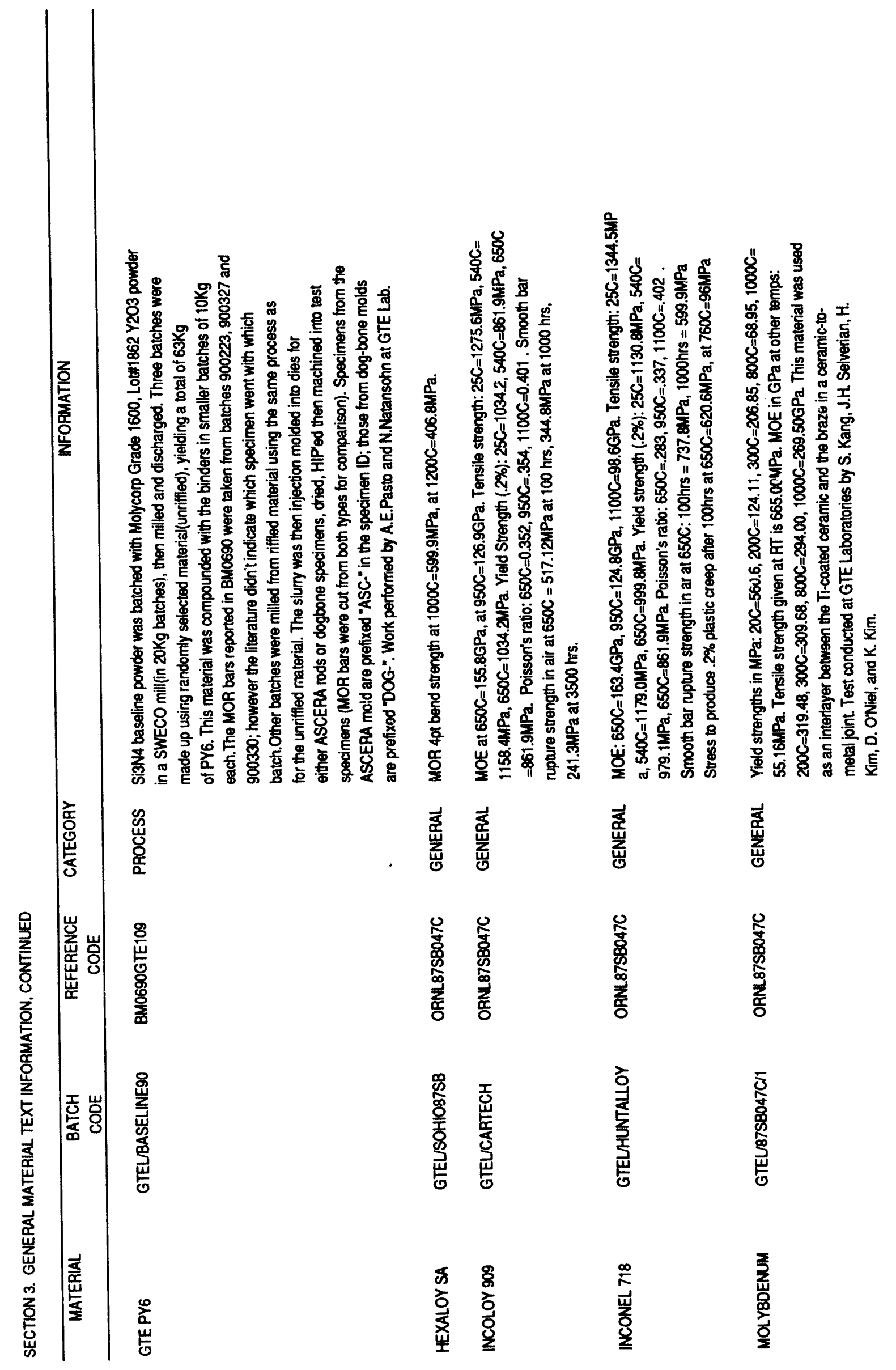




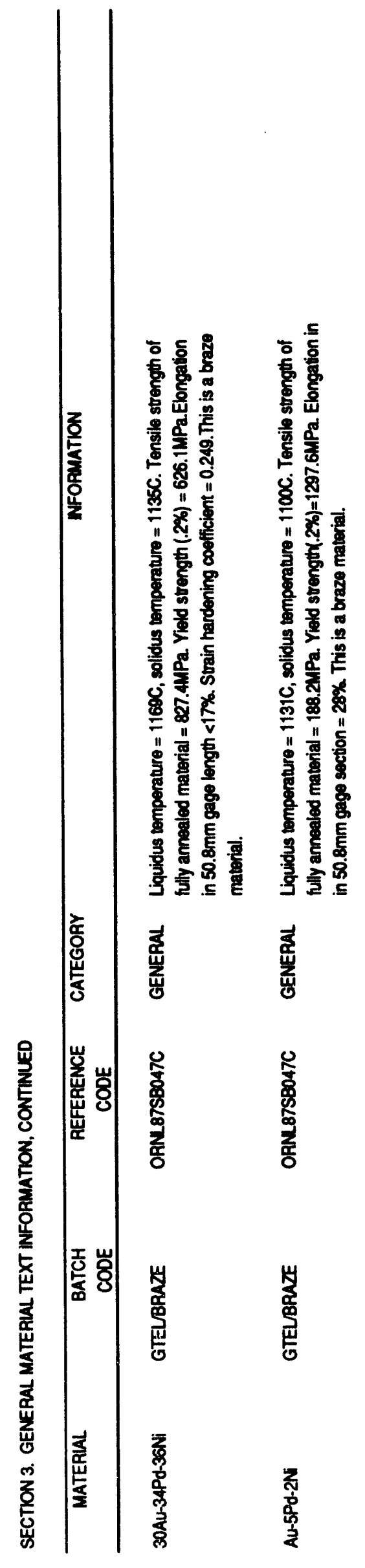




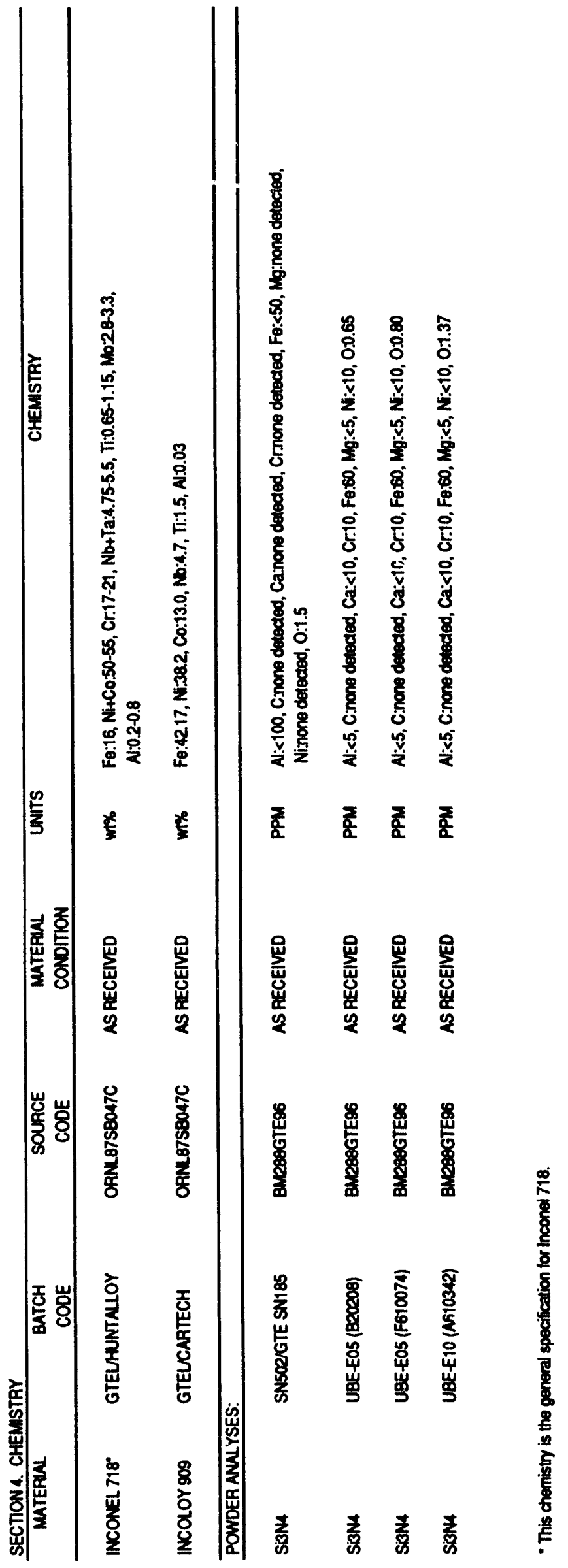




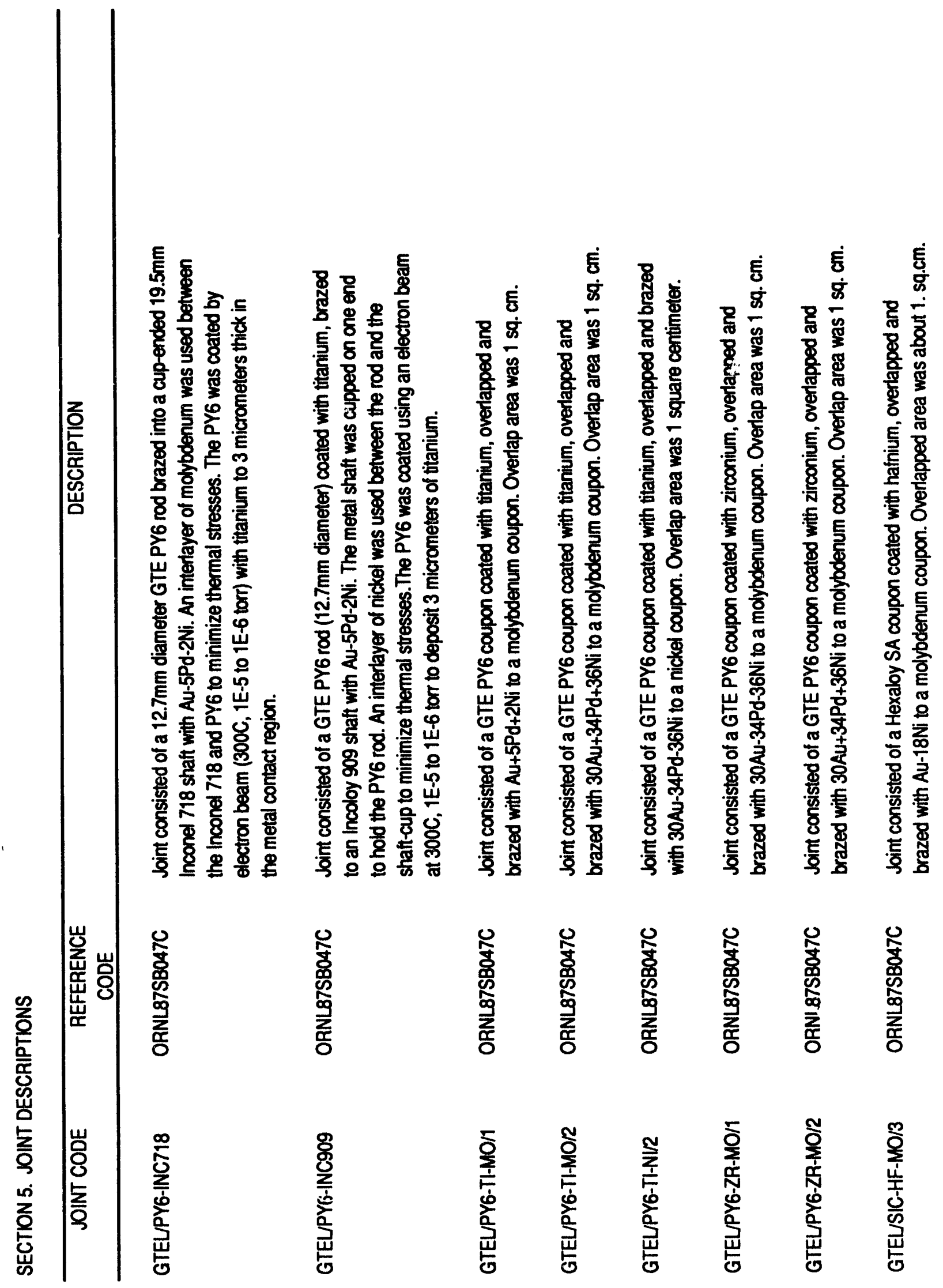




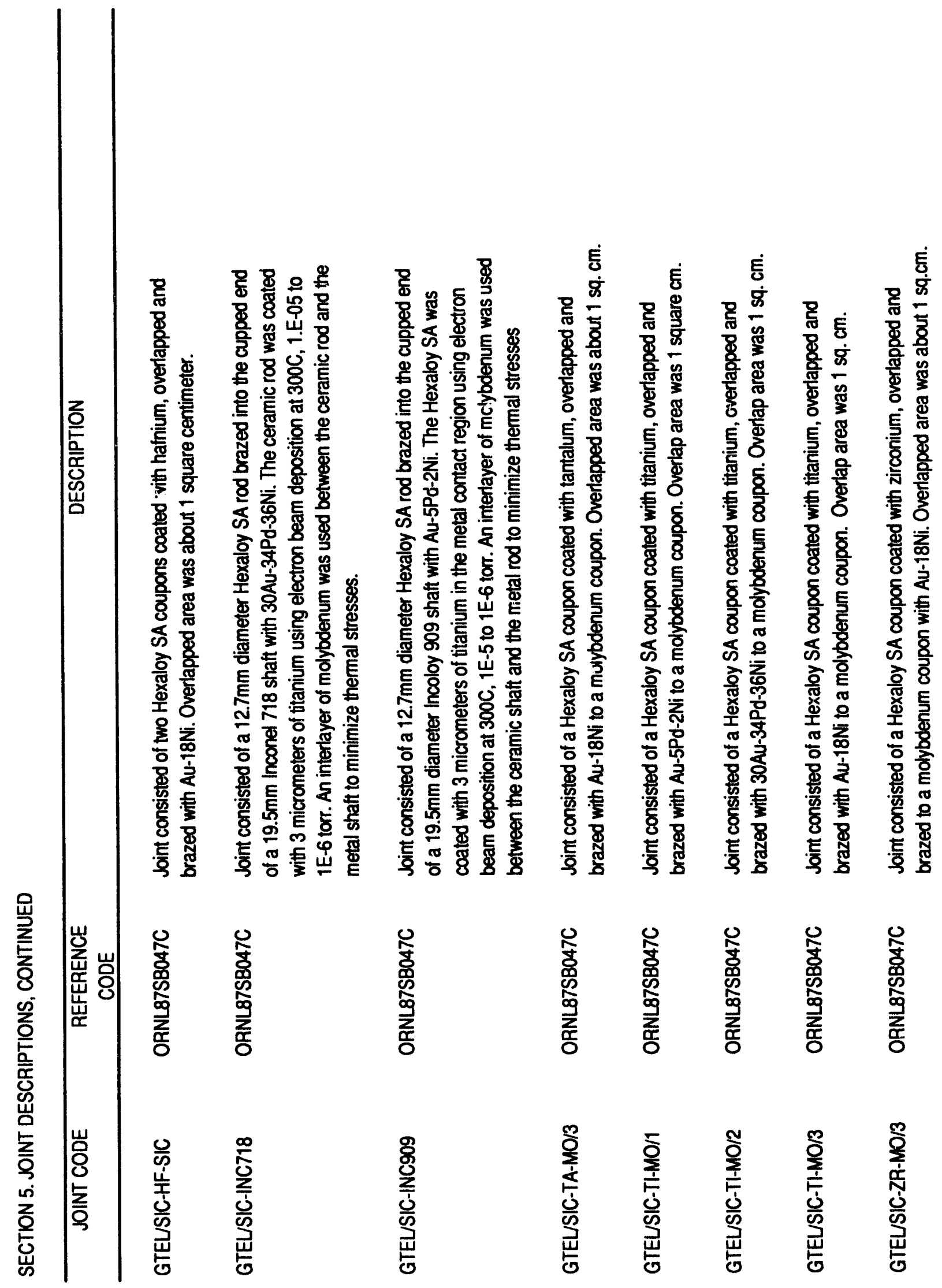




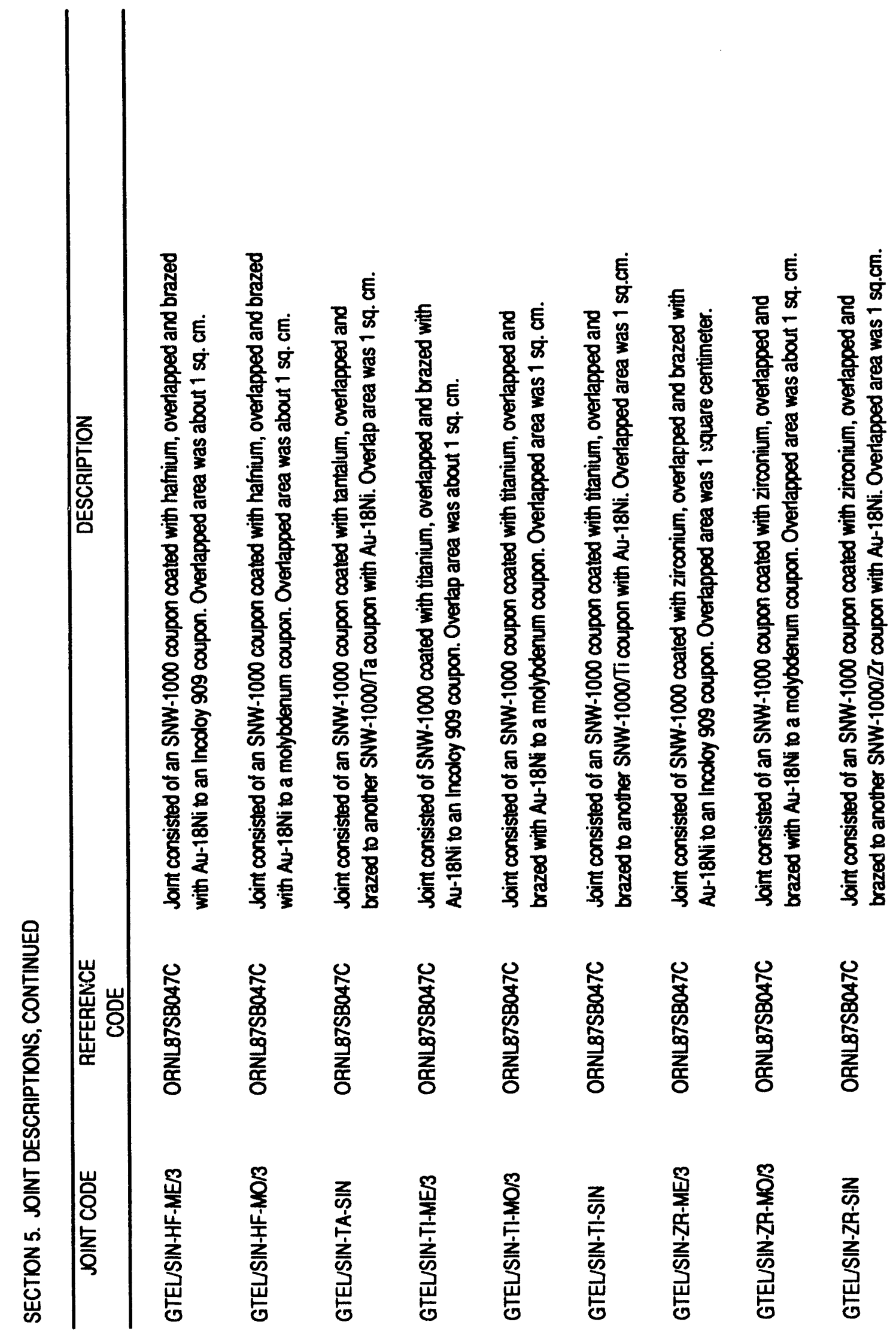




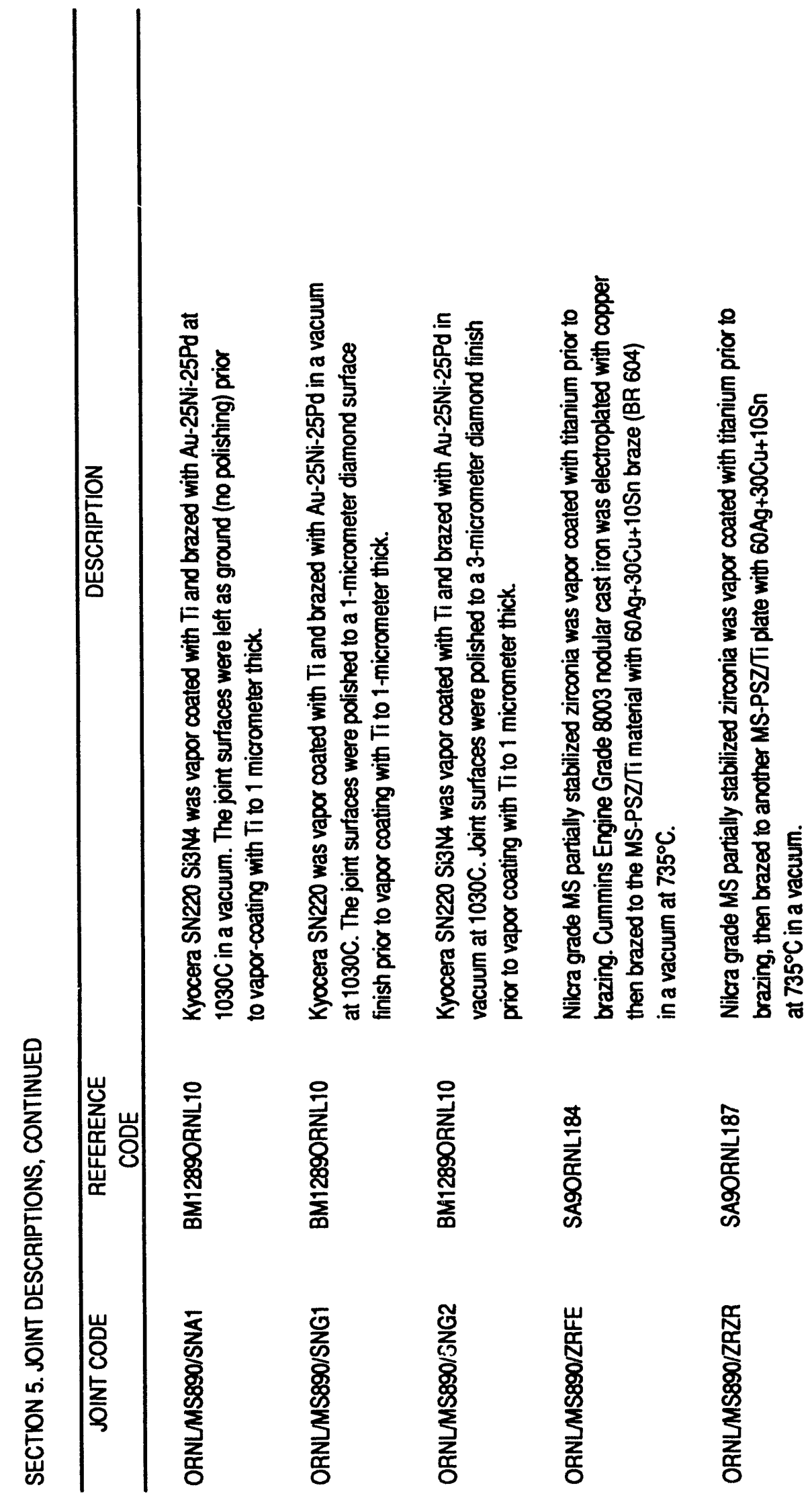


APPENDIXB. TEST RESULTS 


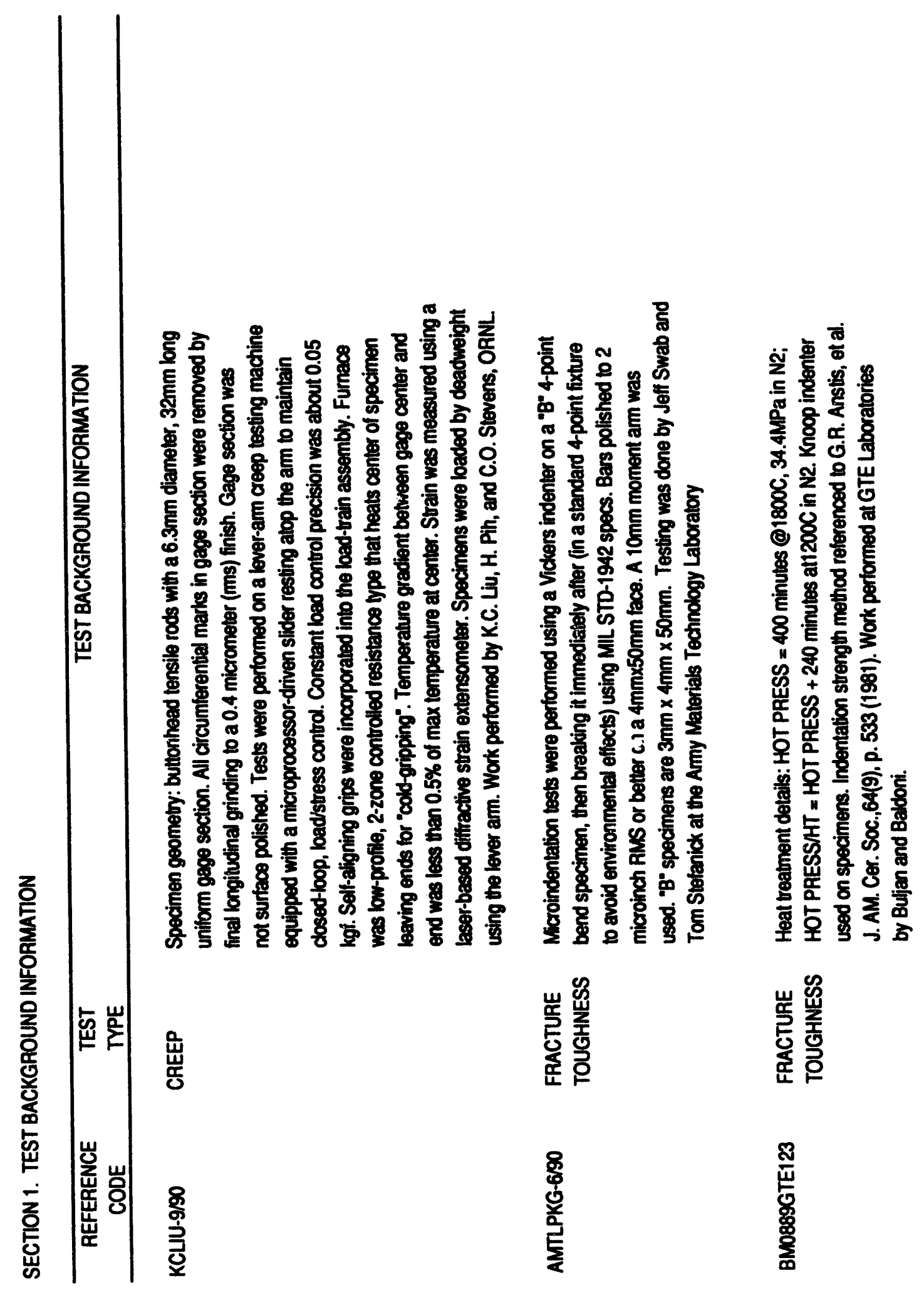




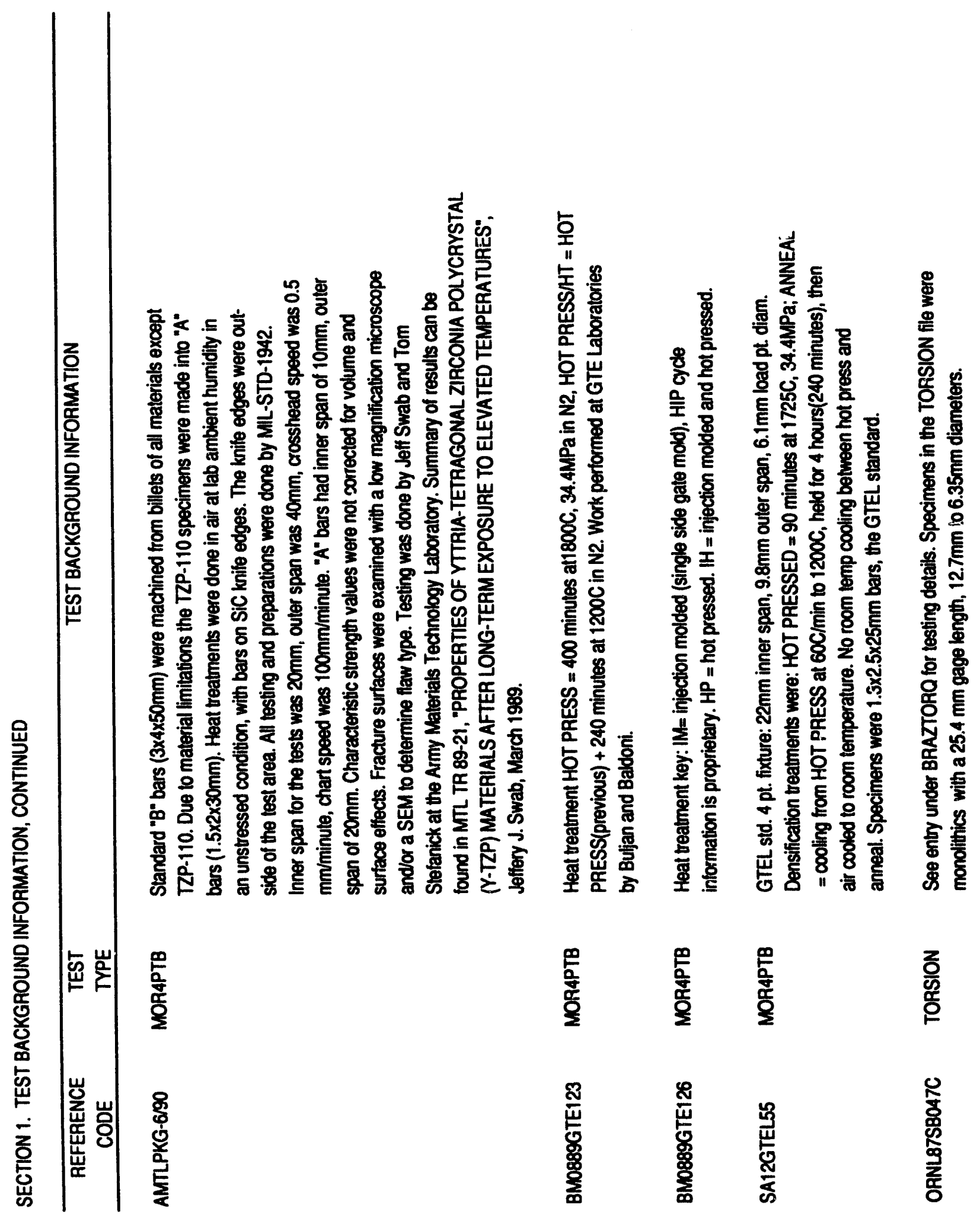




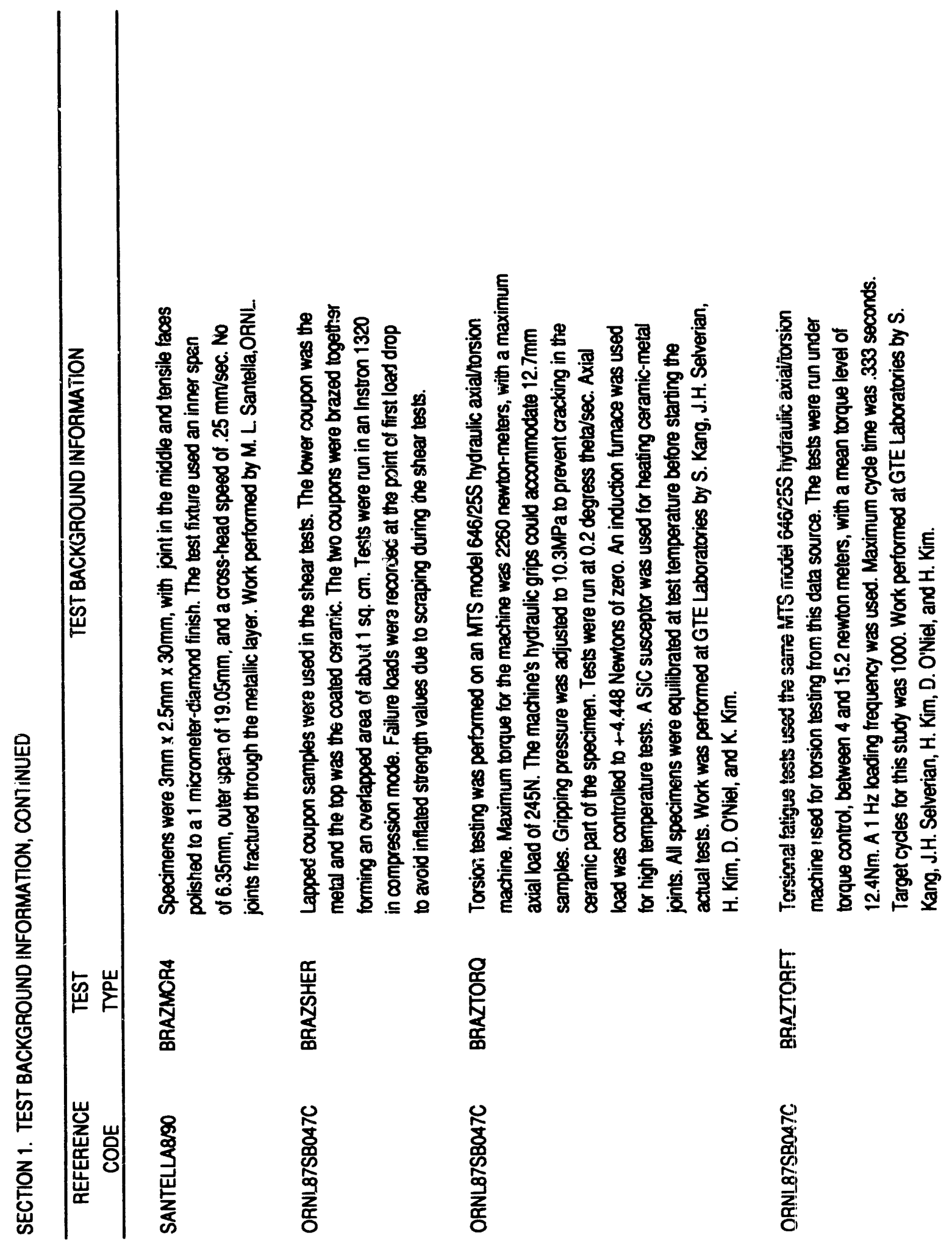




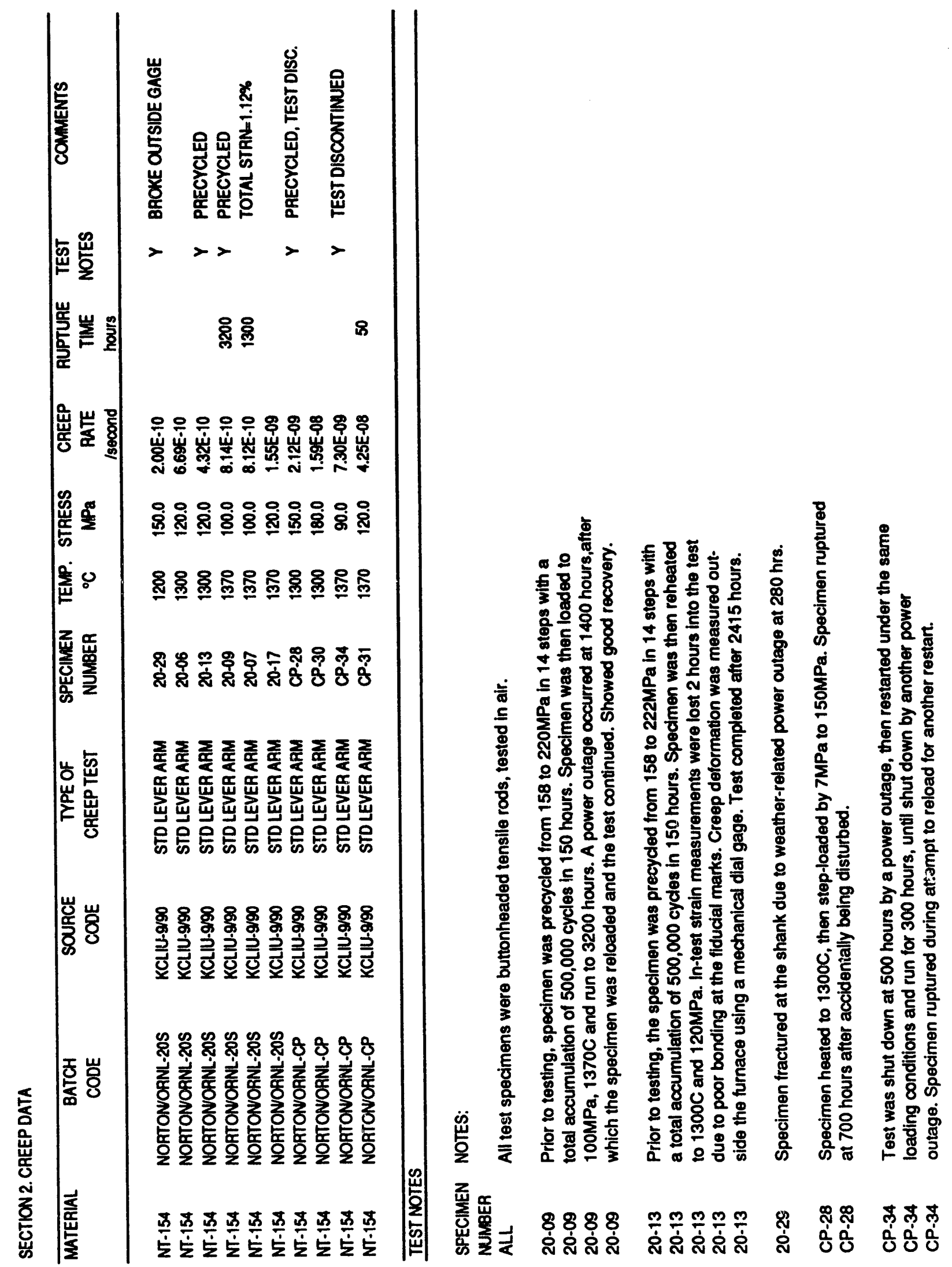




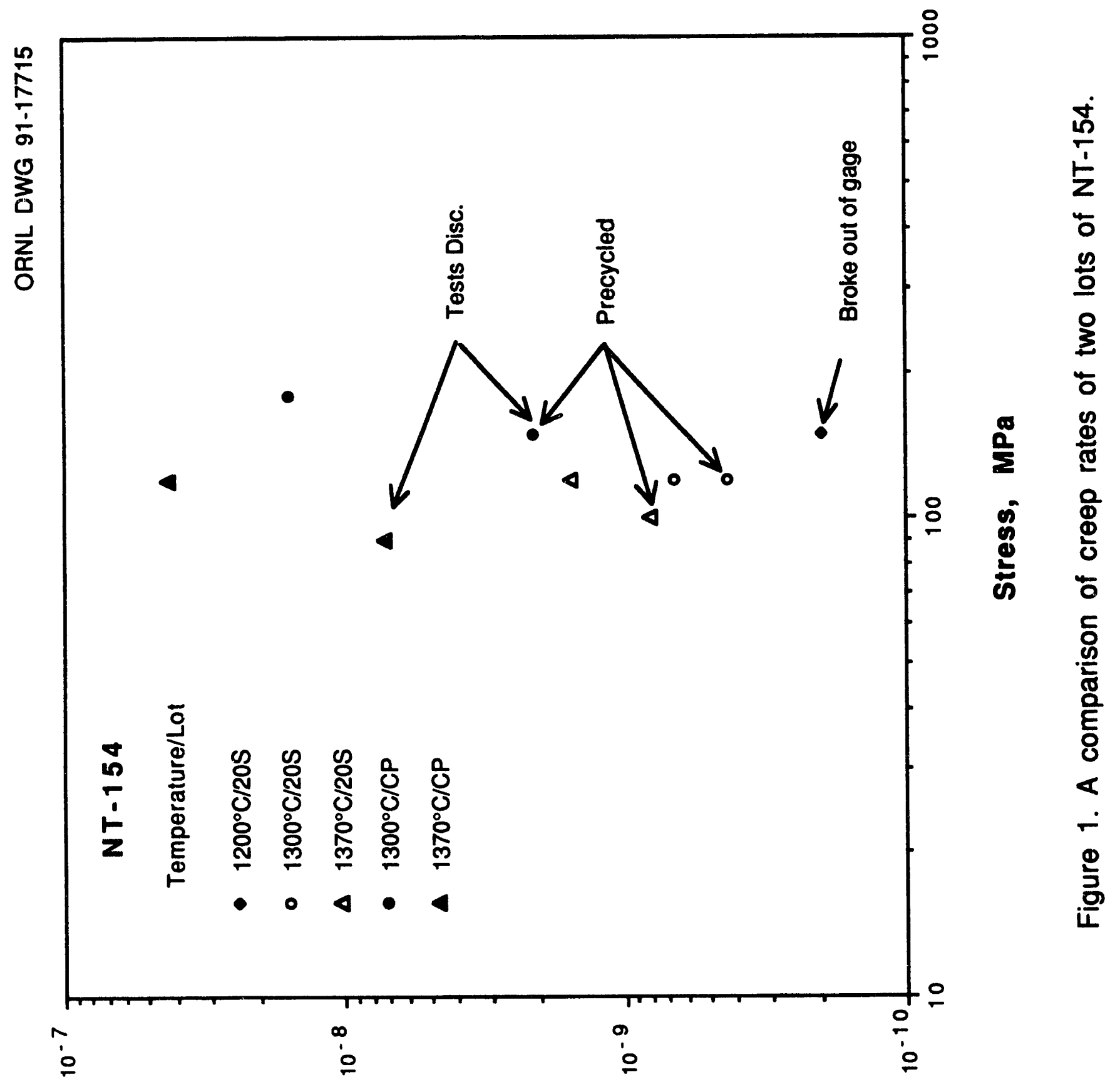

puojes/l 'әرеy dəəدว 


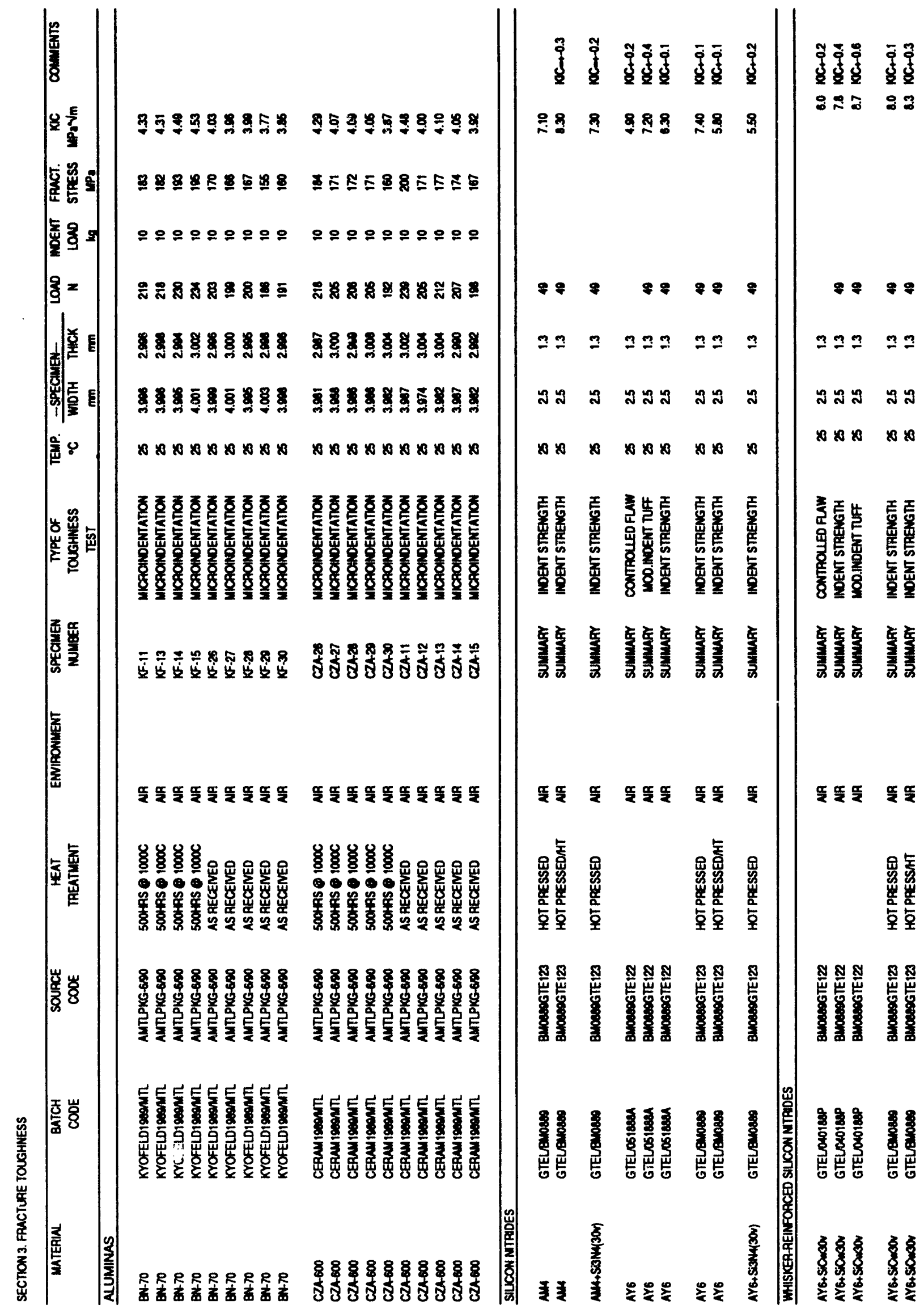




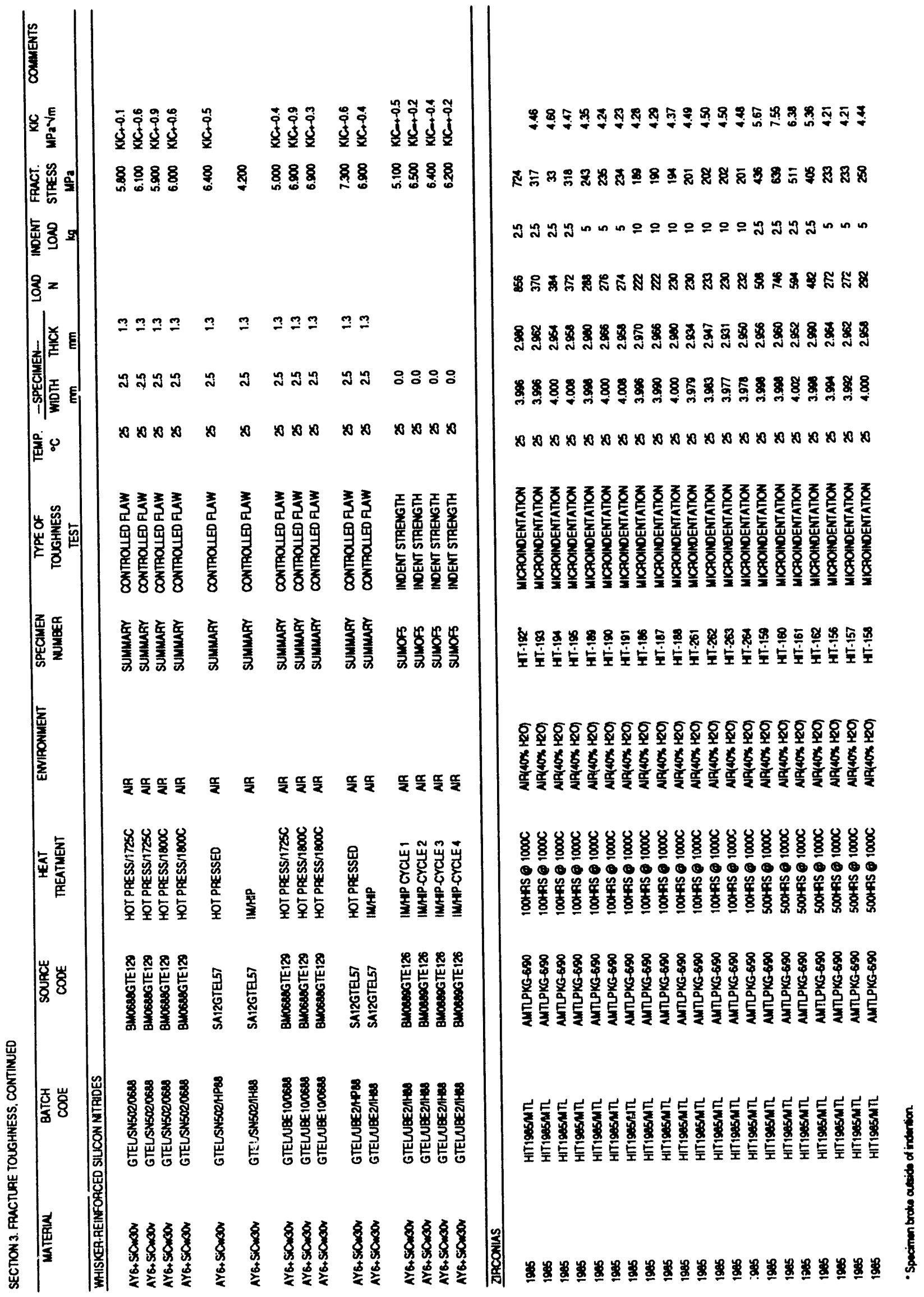




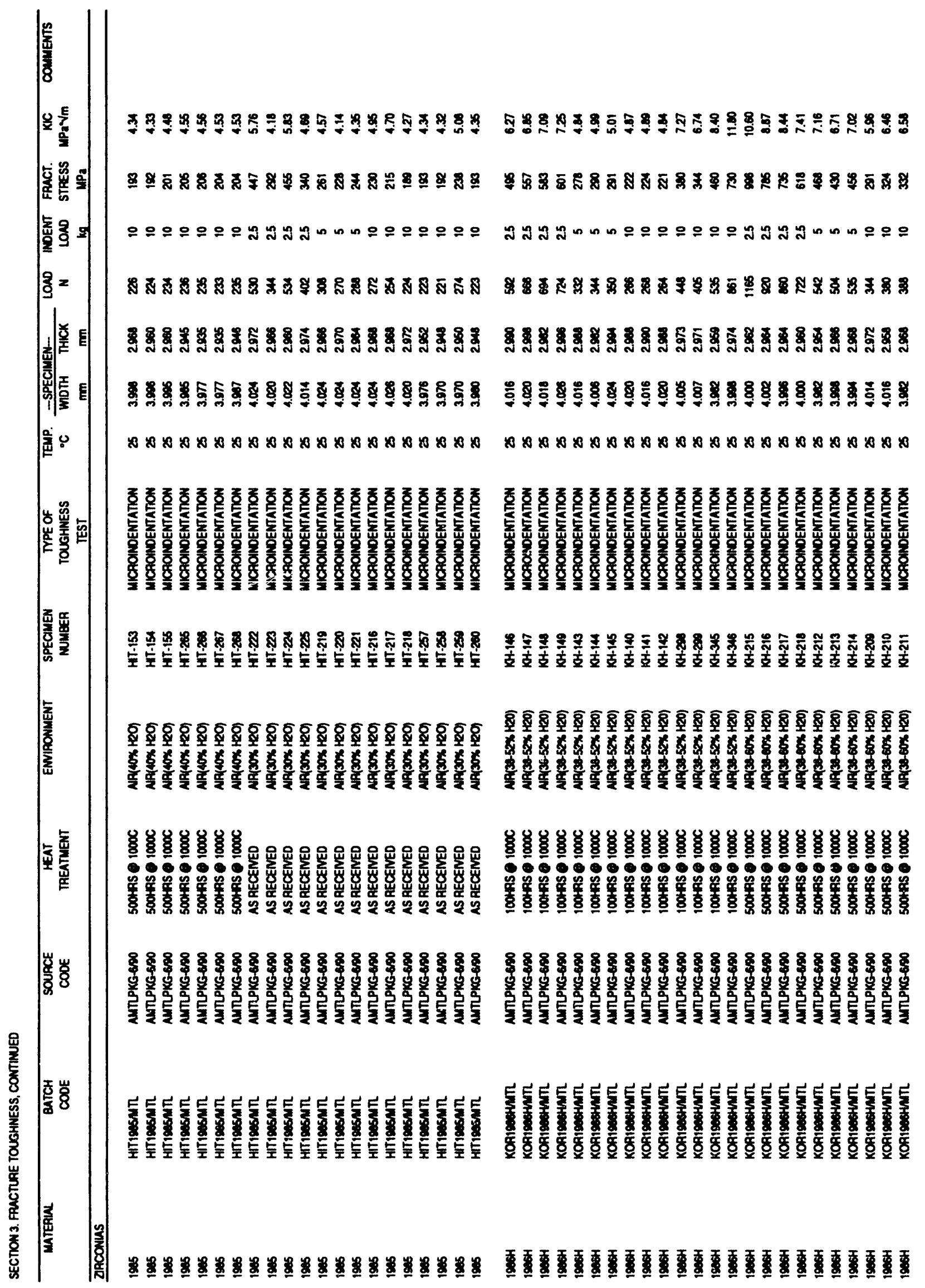




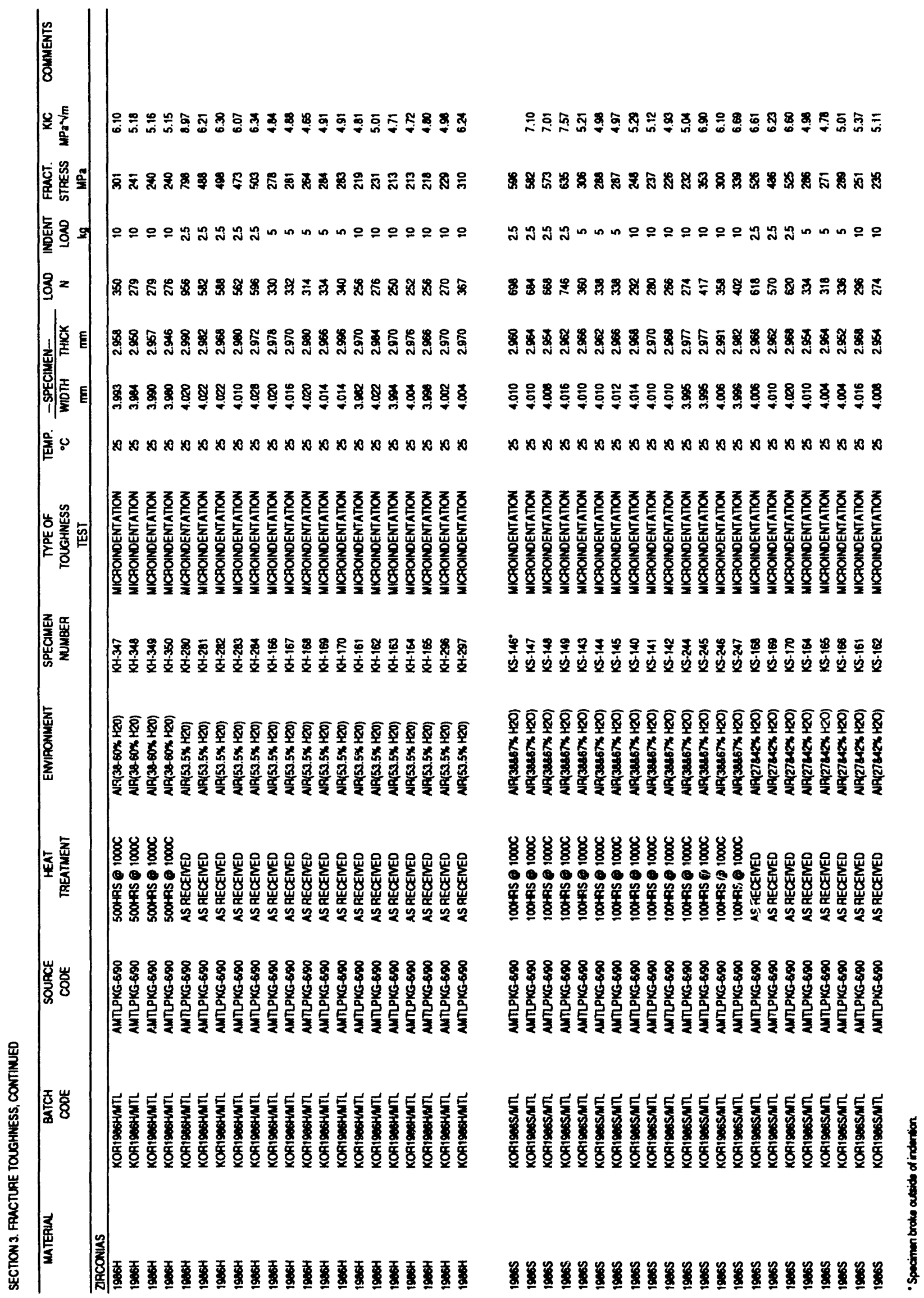




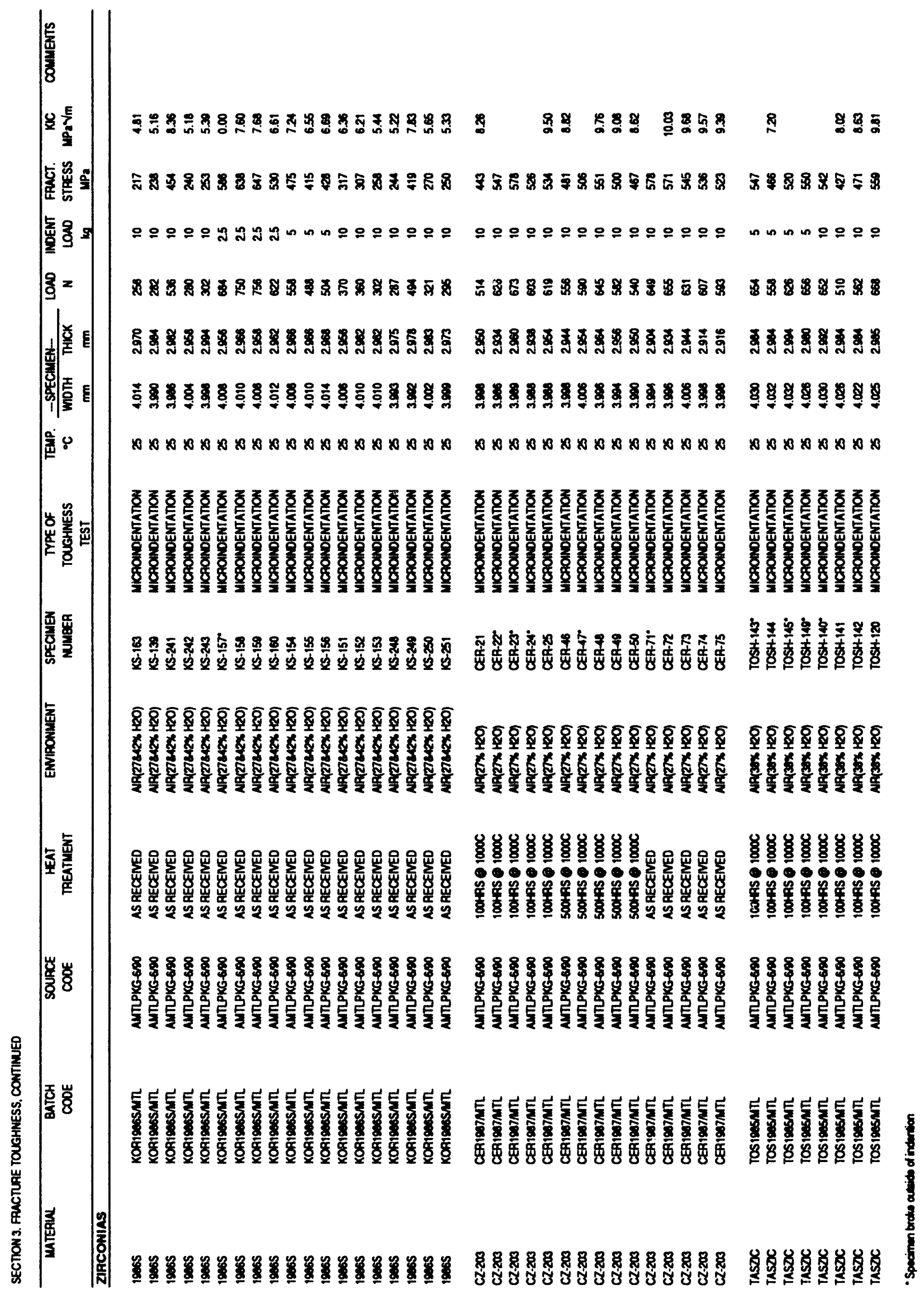




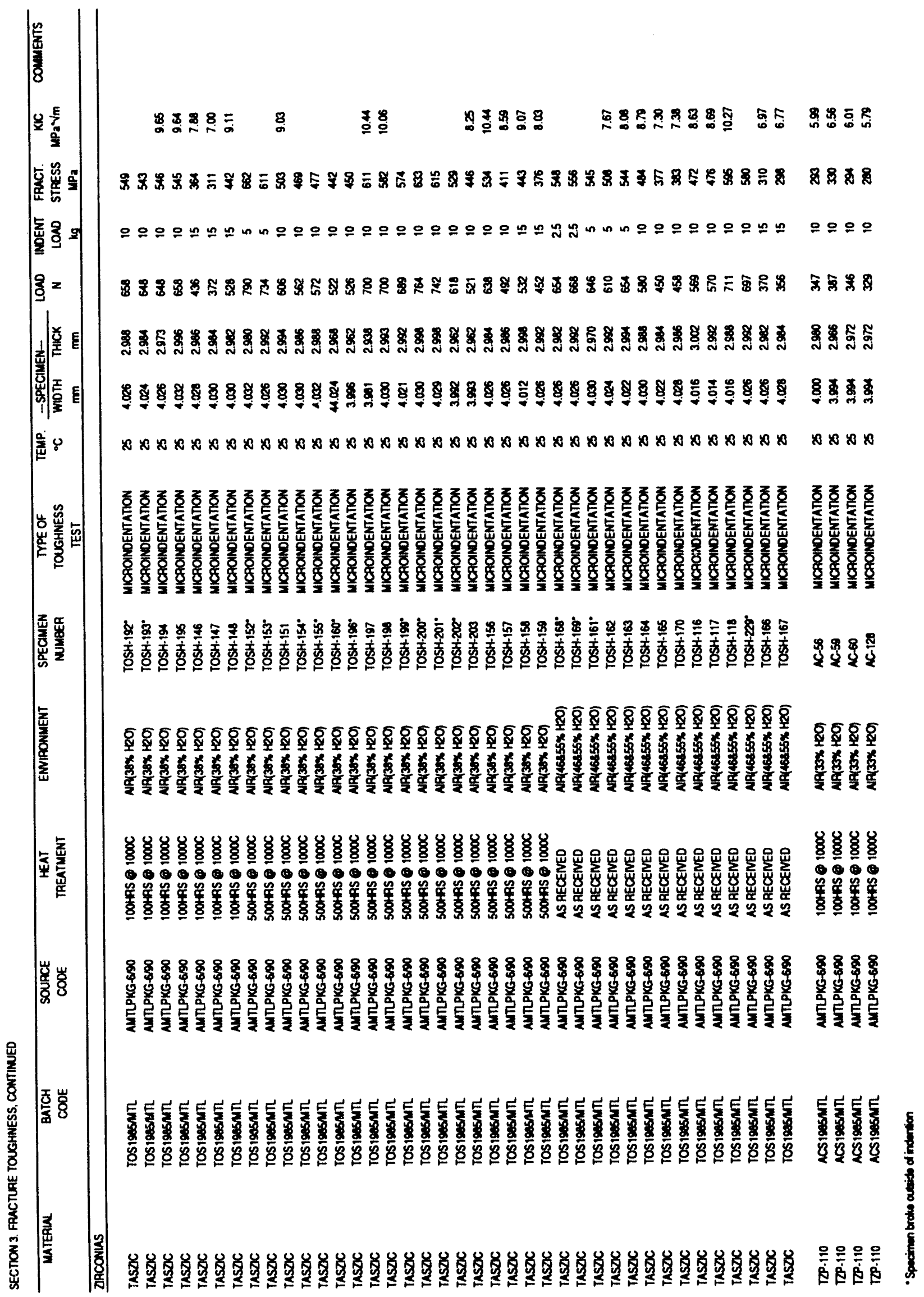




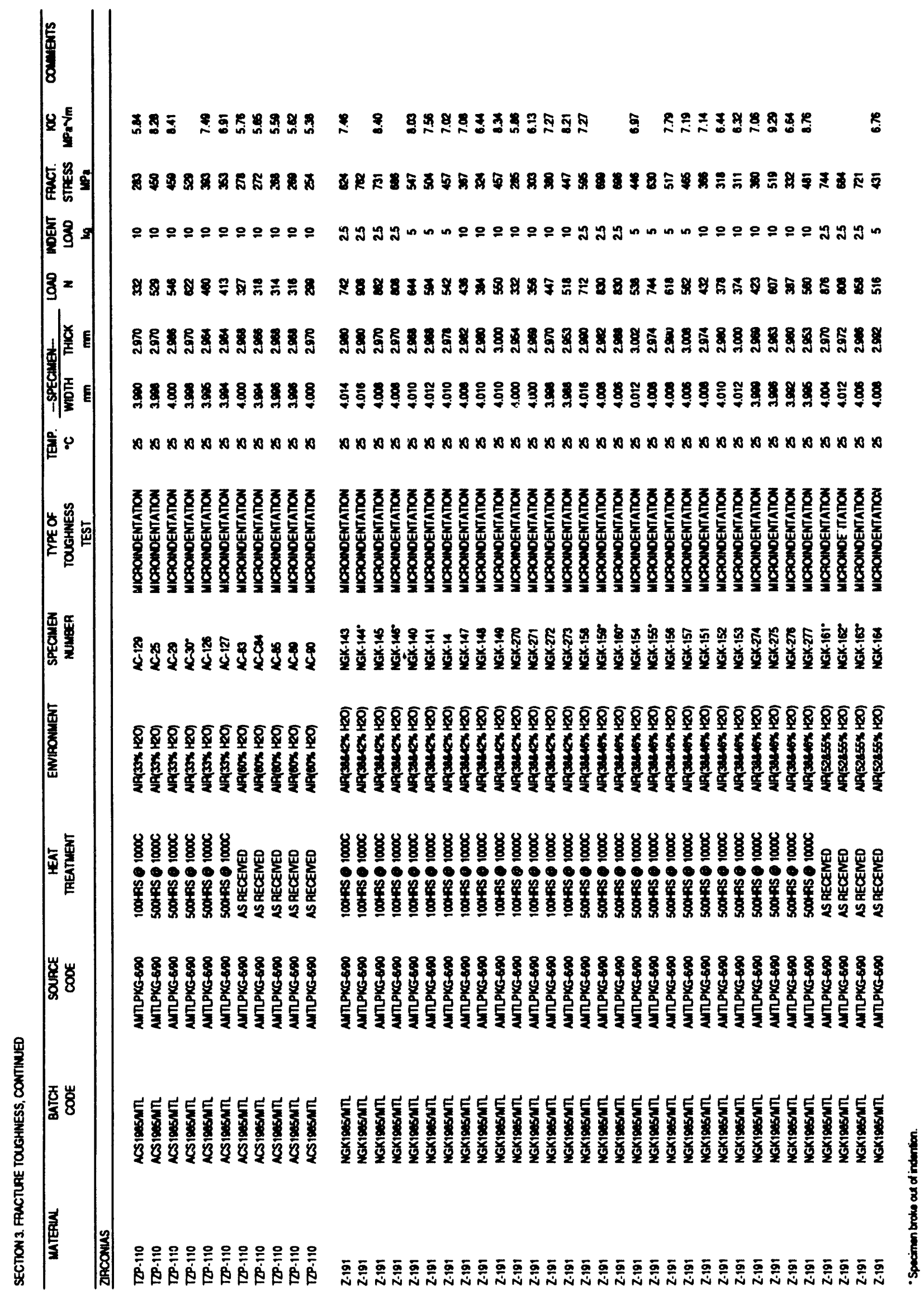




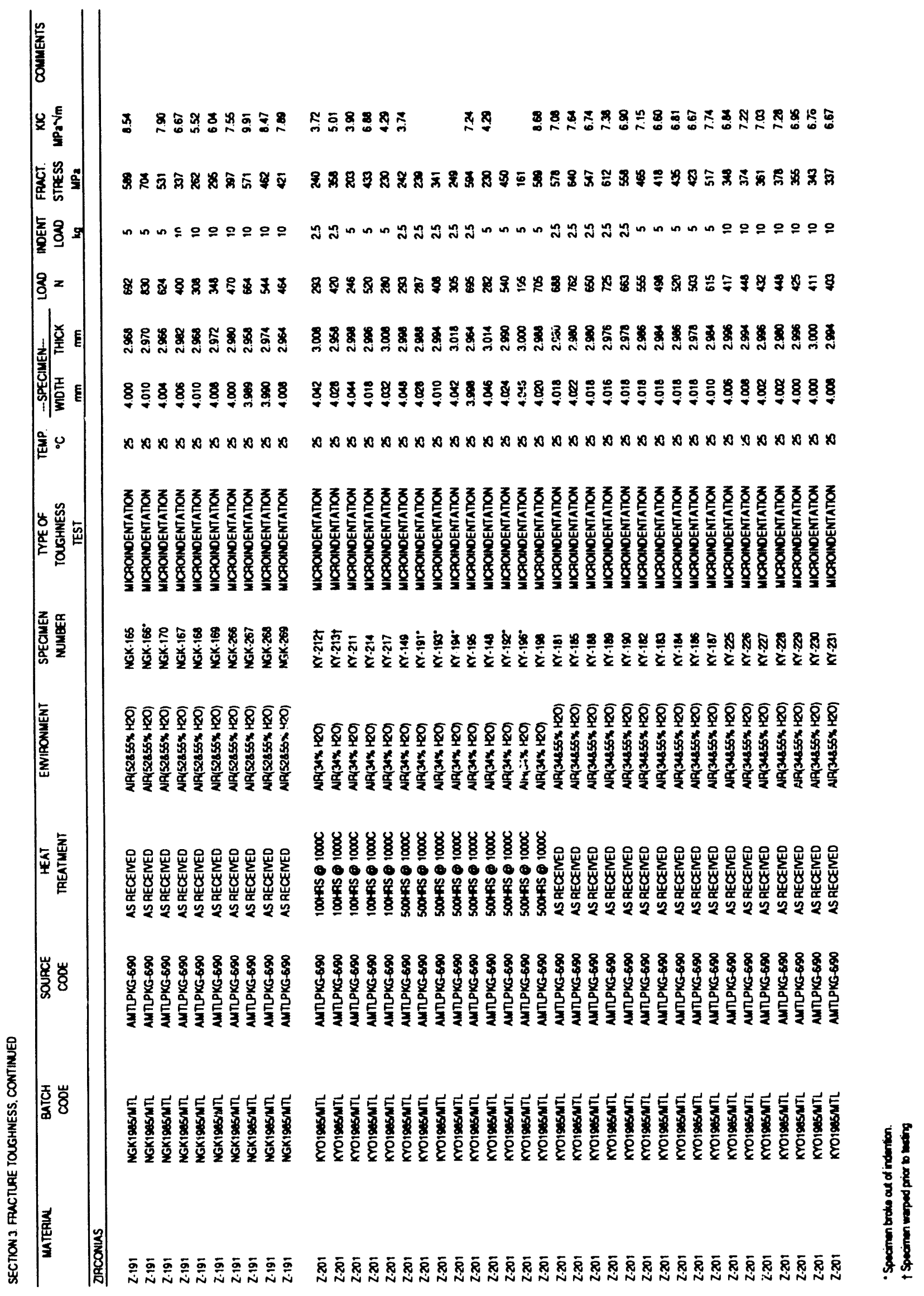




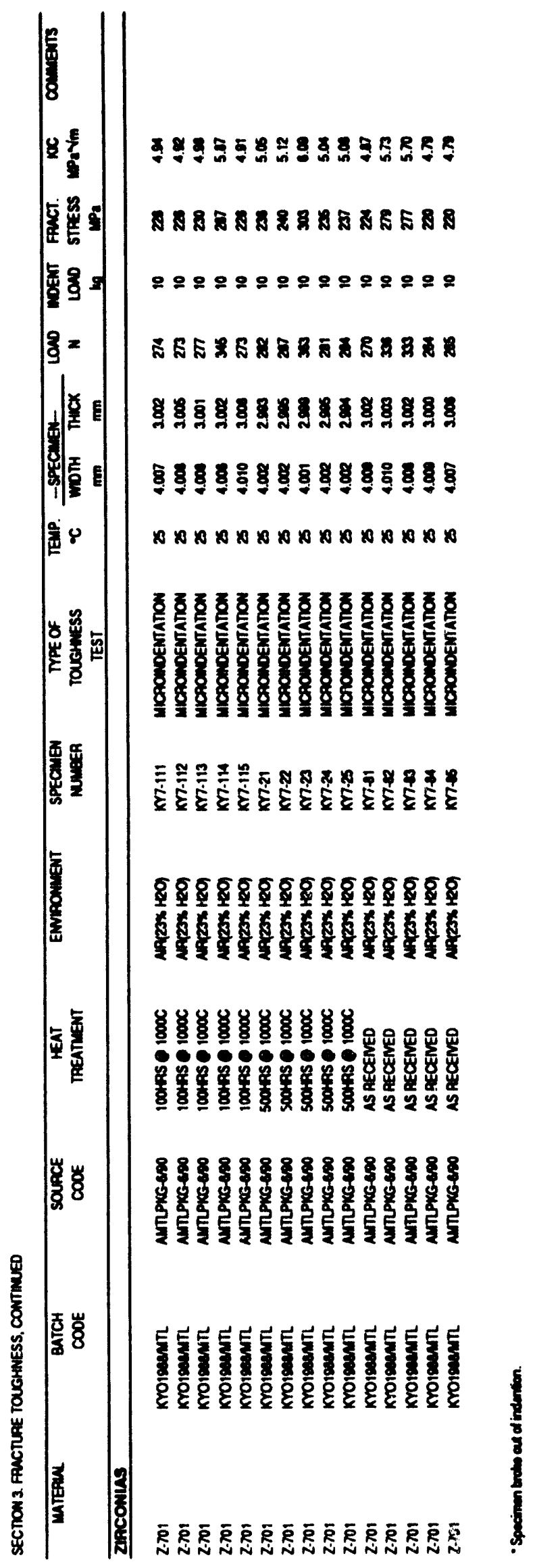




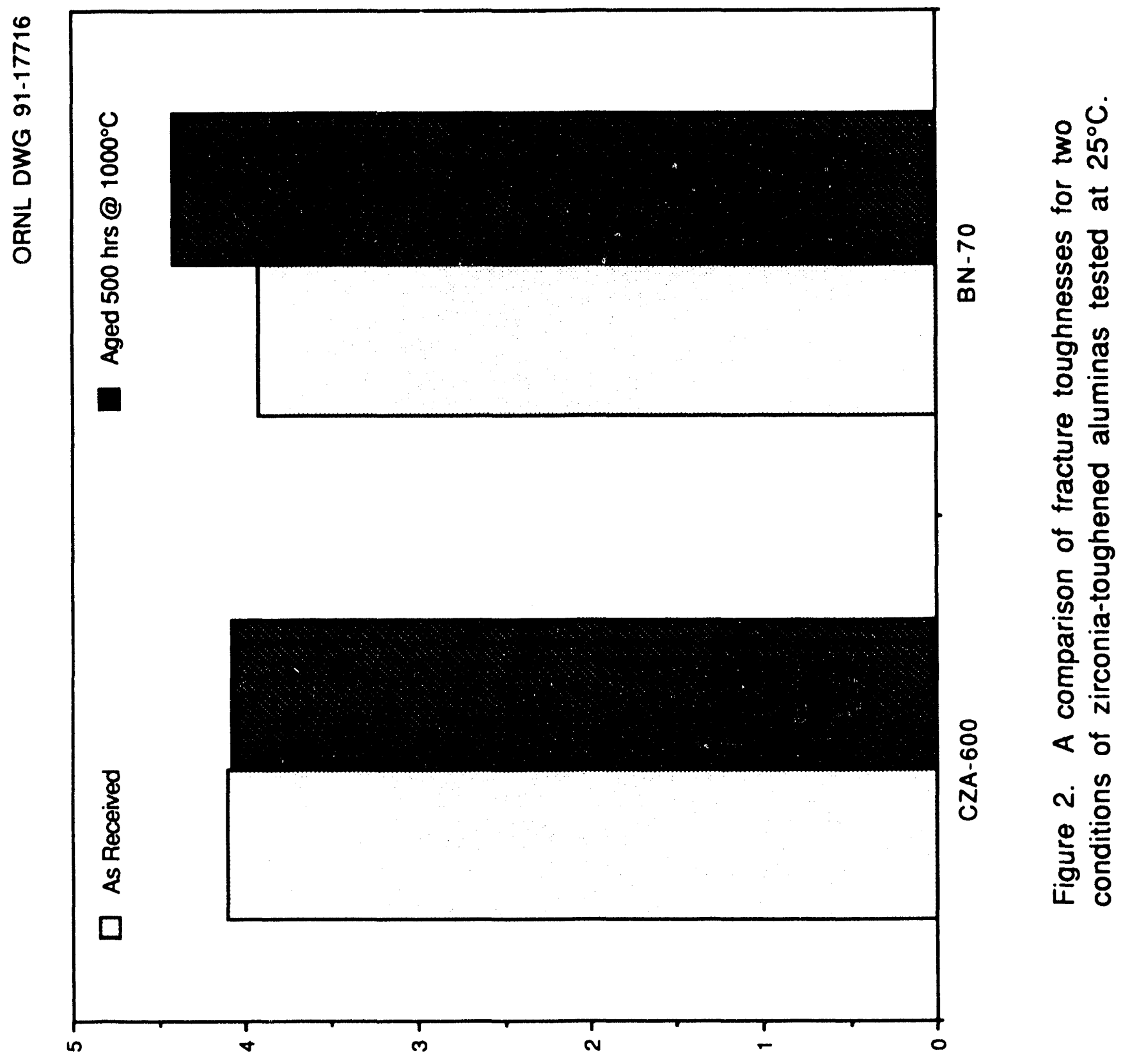

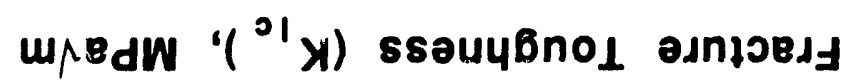


,
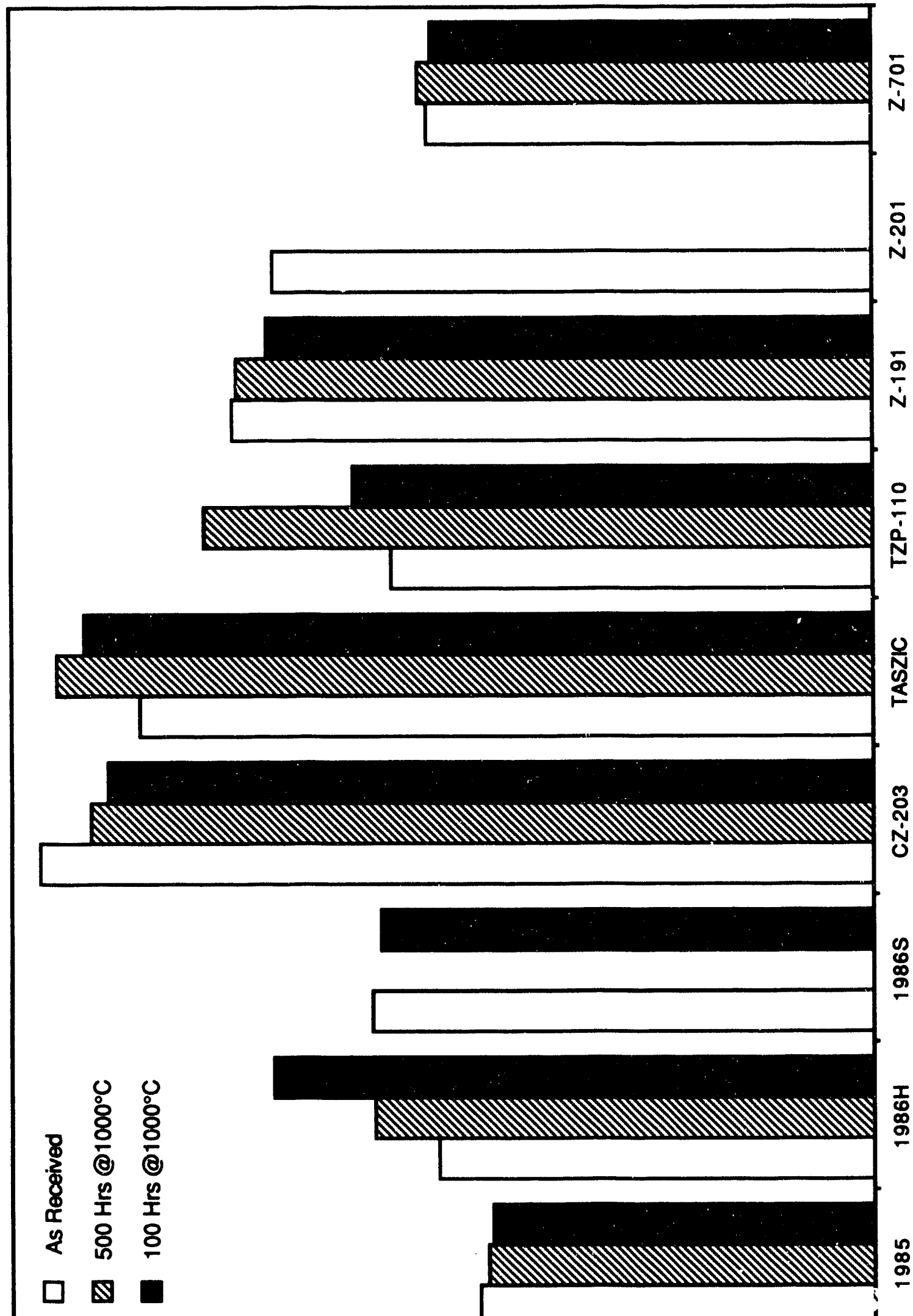

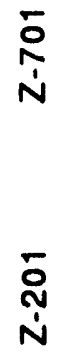

은

$\frac{\bar{\sigma}}{\dot{N}}$

$\dot{N}$

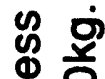

Ф 응

도

옹 온

뽈 융

खิ

농

잋

둥

동 옹

을

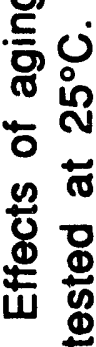

i

Ф 을

농어

은 읏

I
\$

$-$

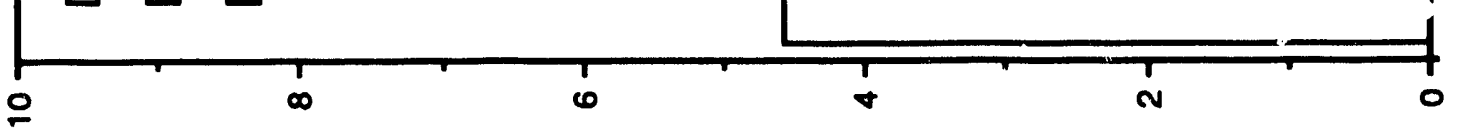

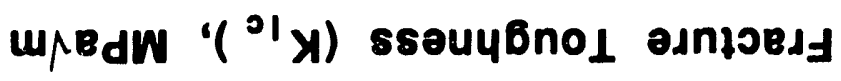




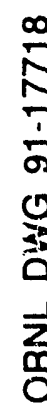

赔

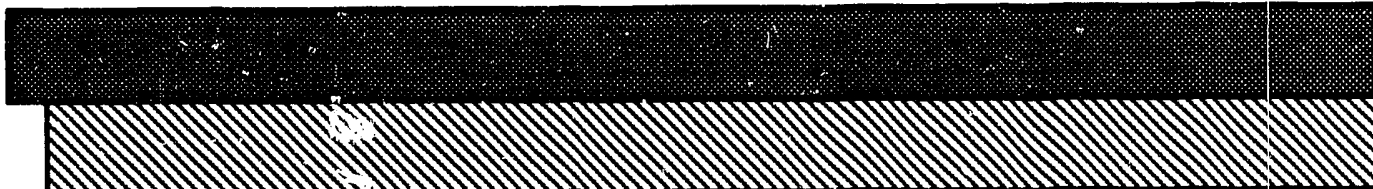

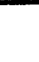

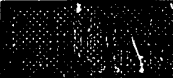

용ㅇㅇㅇㅇㅇㅇ

()

늘 \&

응 용

- $\mathbb{1}$

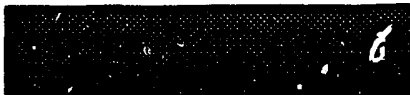

.
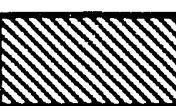

11112
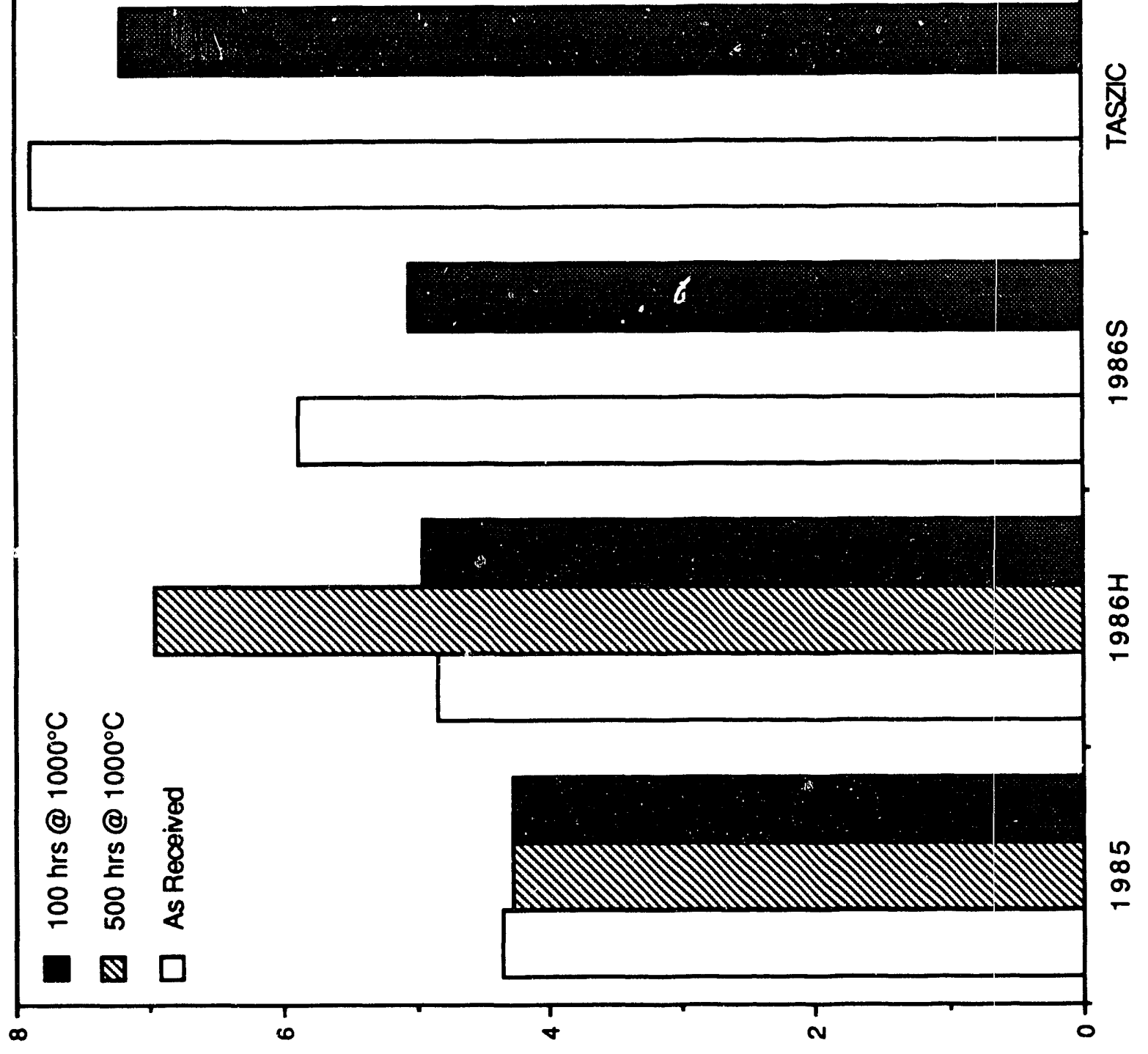

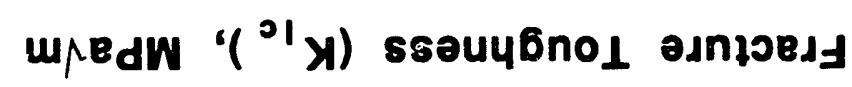

$\frac{\frac{\pi}{E}}{\frac{8}{N}}$

$\bar{x}$

क

ठั

ธ

으

os

옹

동

잉

蒡

๘.5

壱

$\Phi$

10

ธᄃ ํํำ

ำ

产

웡

这

แ.

ष

일 


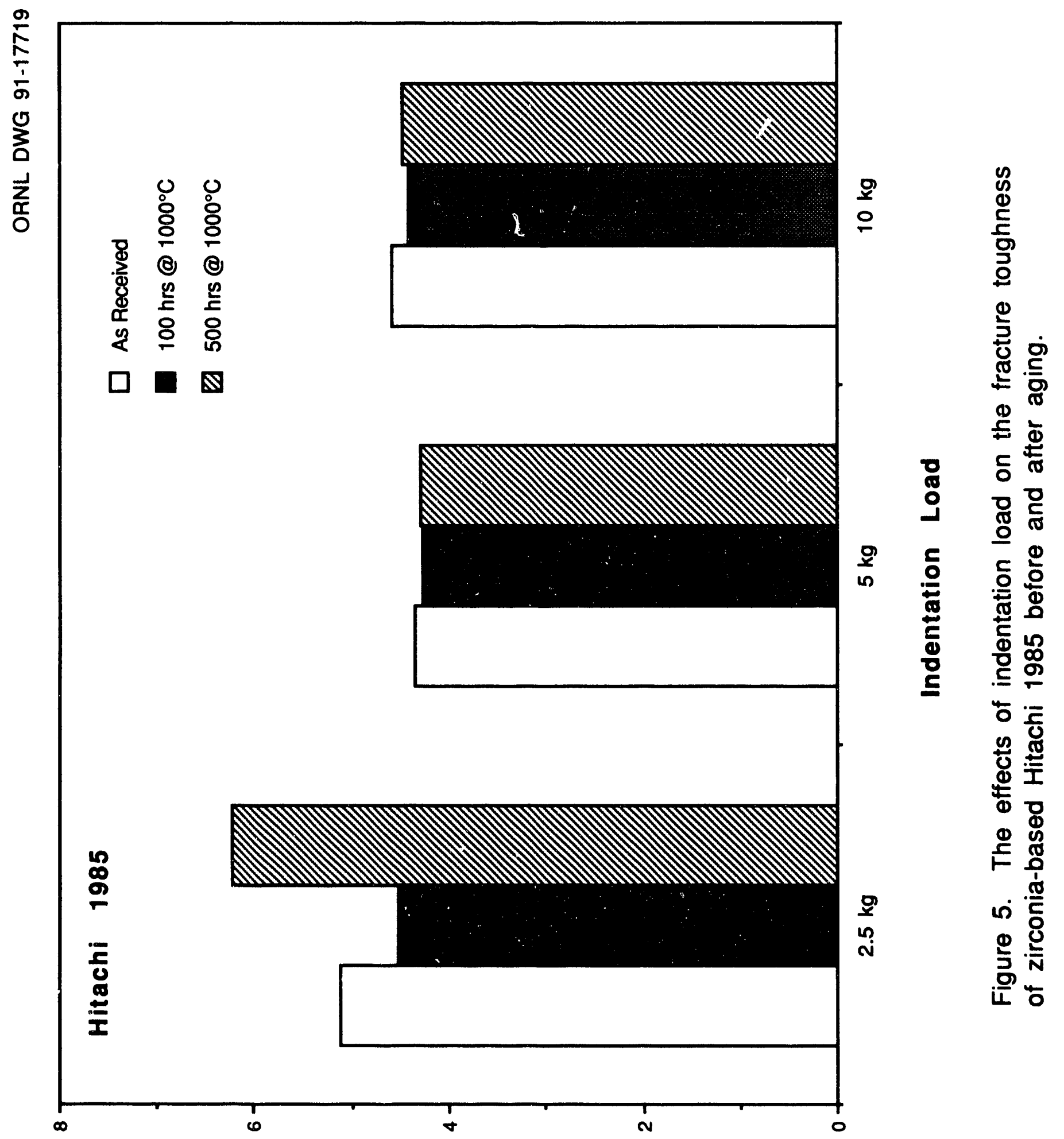

mpedw " $\left({ }^{\circ} x\right)$ ssouy6nol oגnłordy 

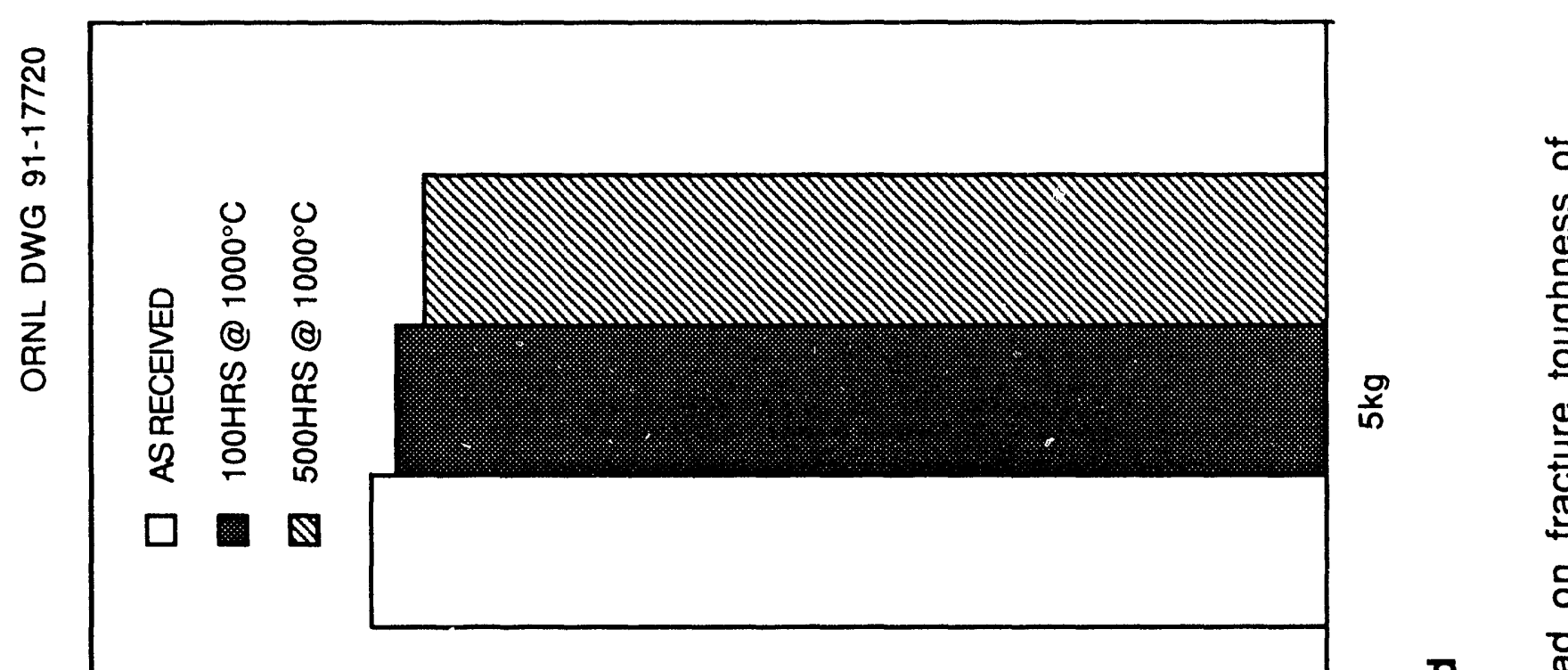

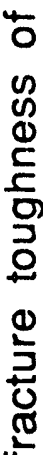

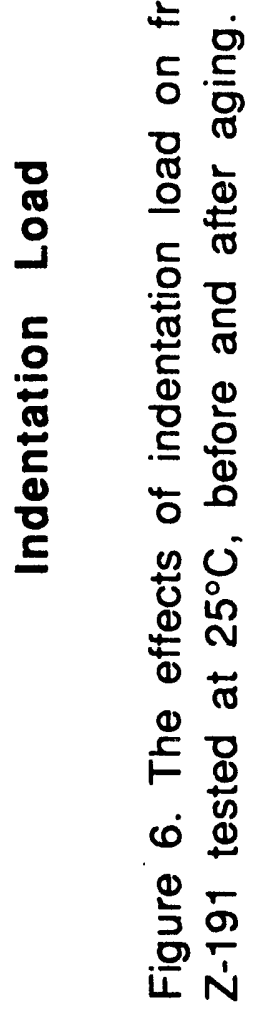

wpedw '( ${ }^{\prime}(x)$ ssauy6nol oגnioed 


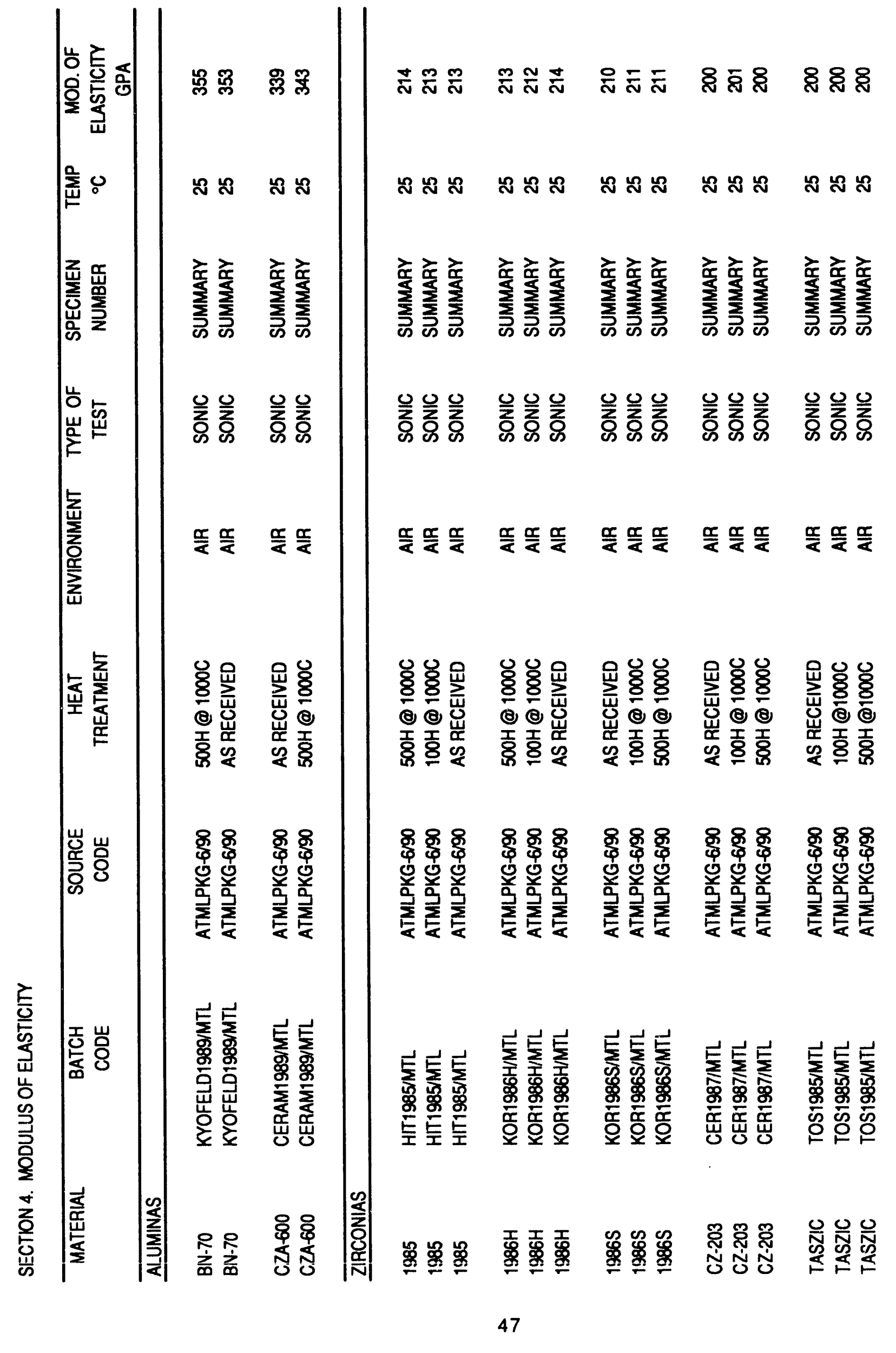




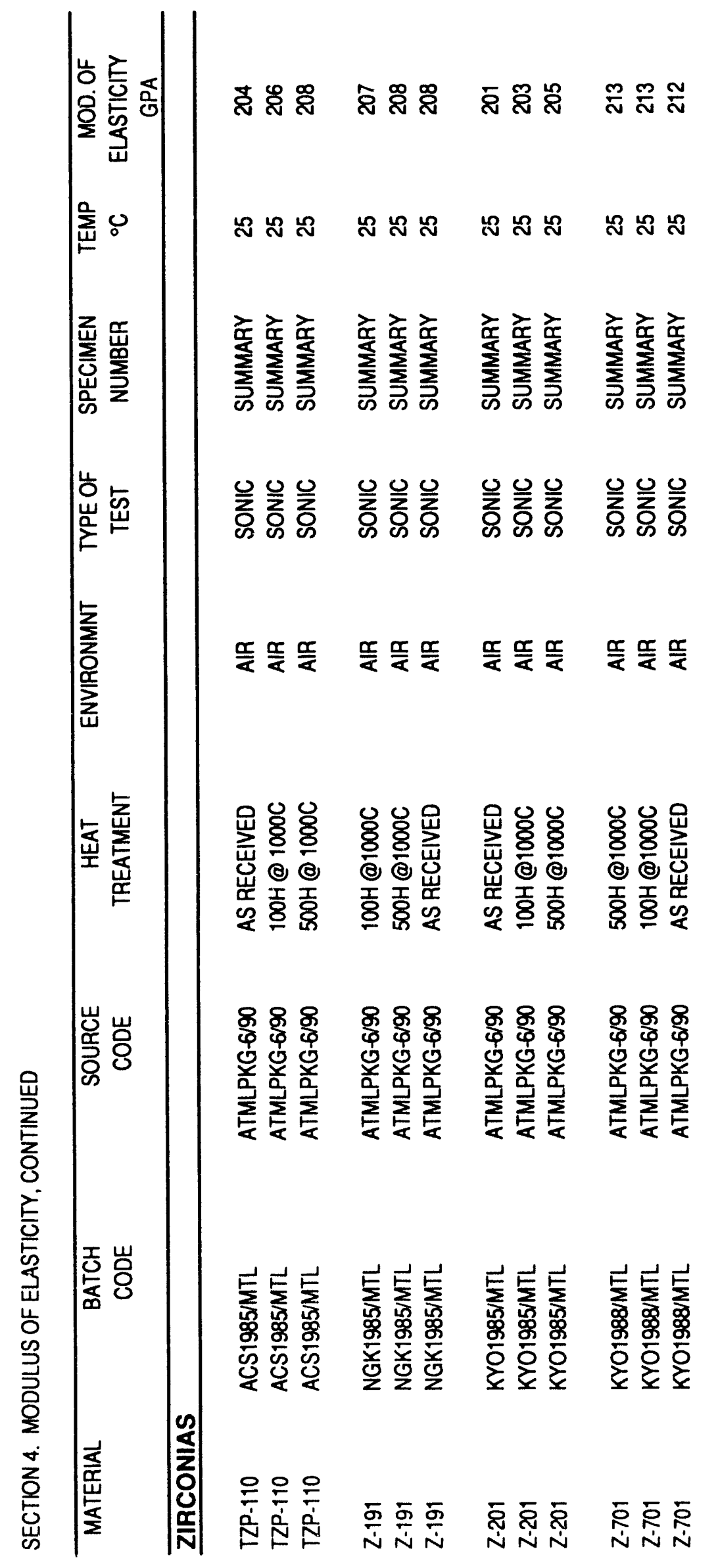




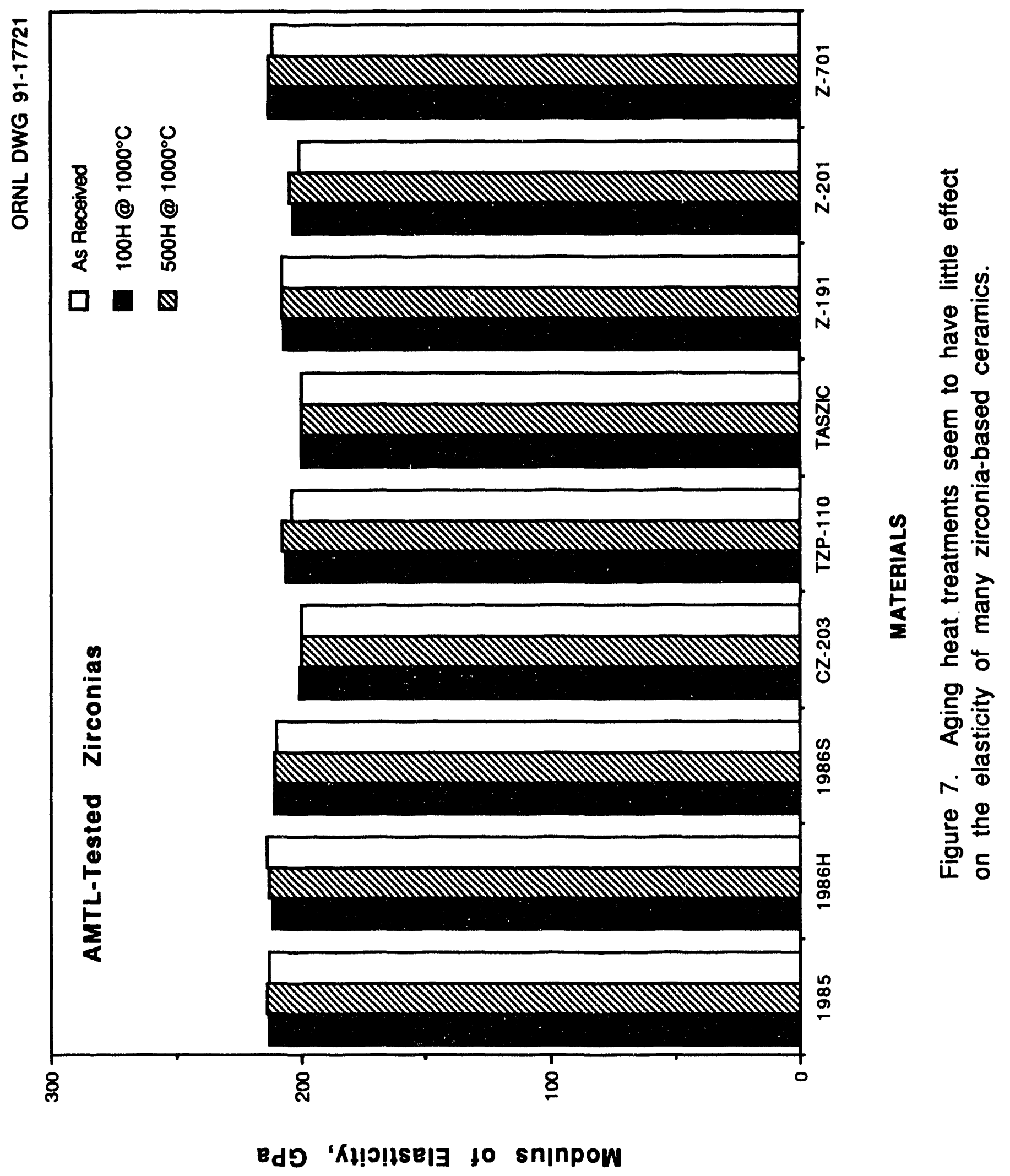




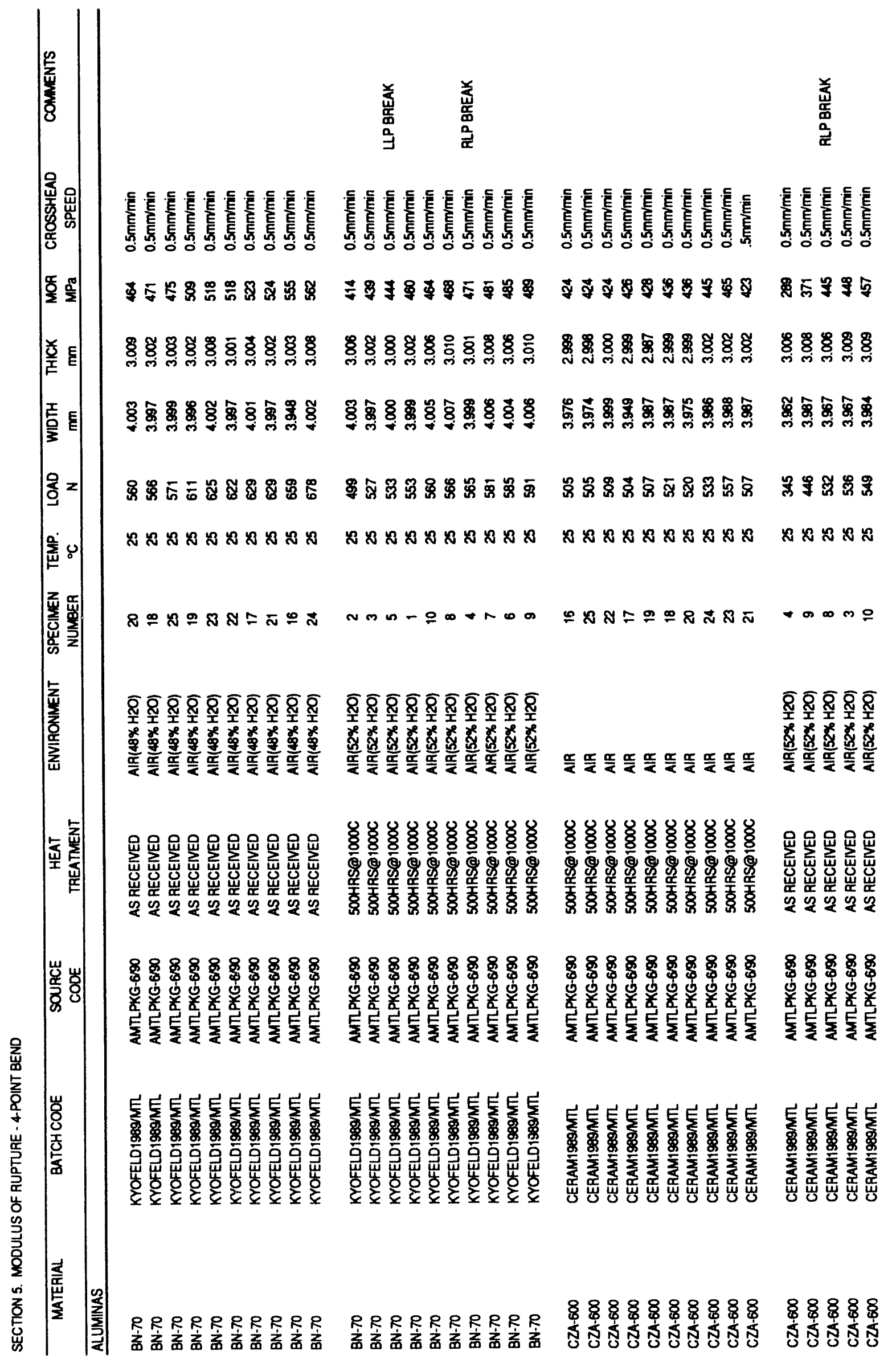




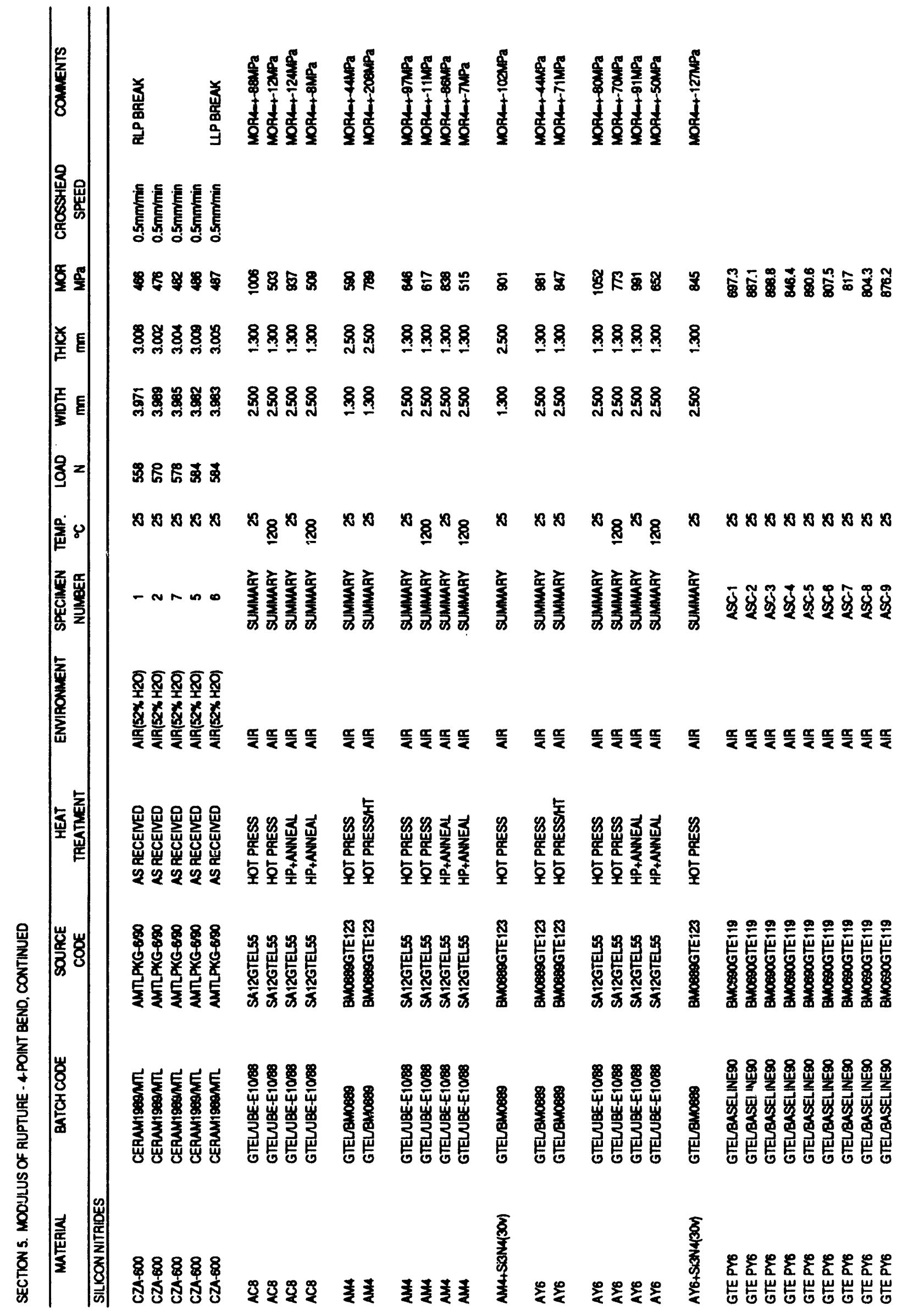




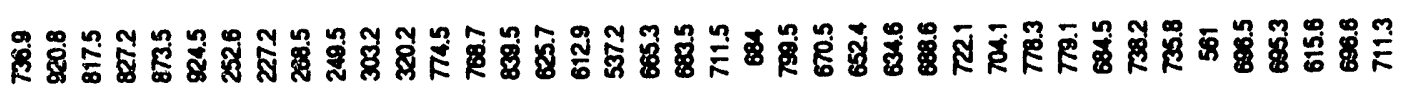

ำ

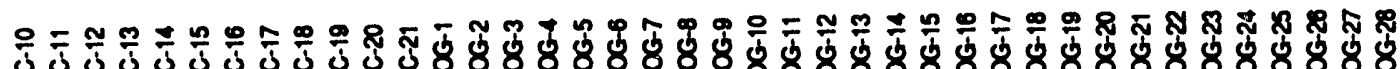

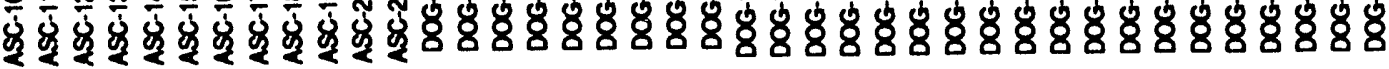

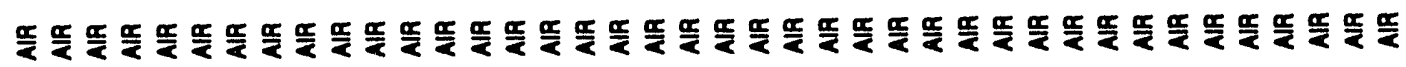

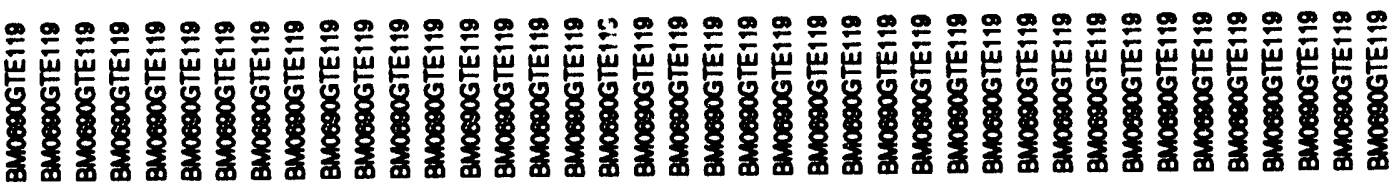

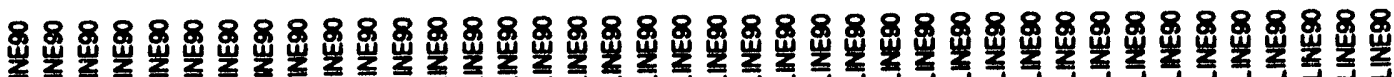

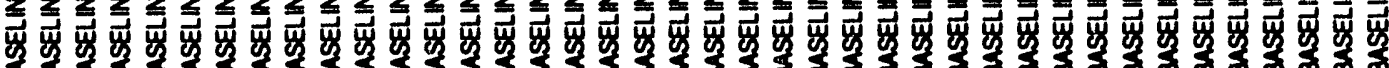

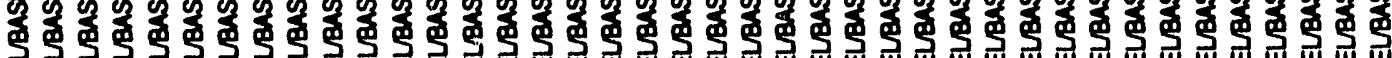

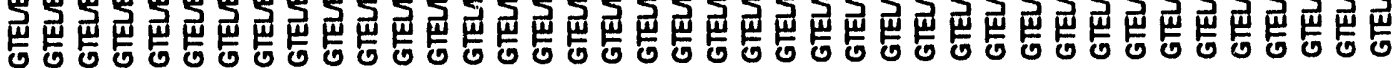

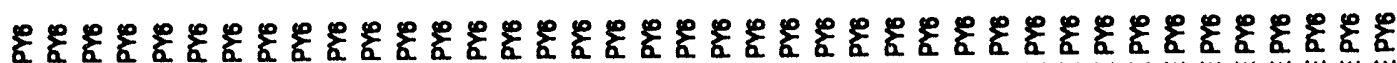

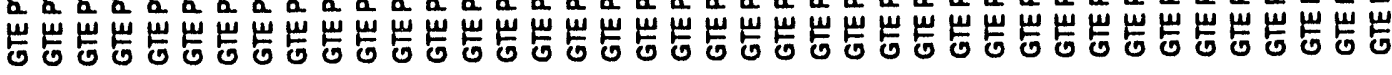




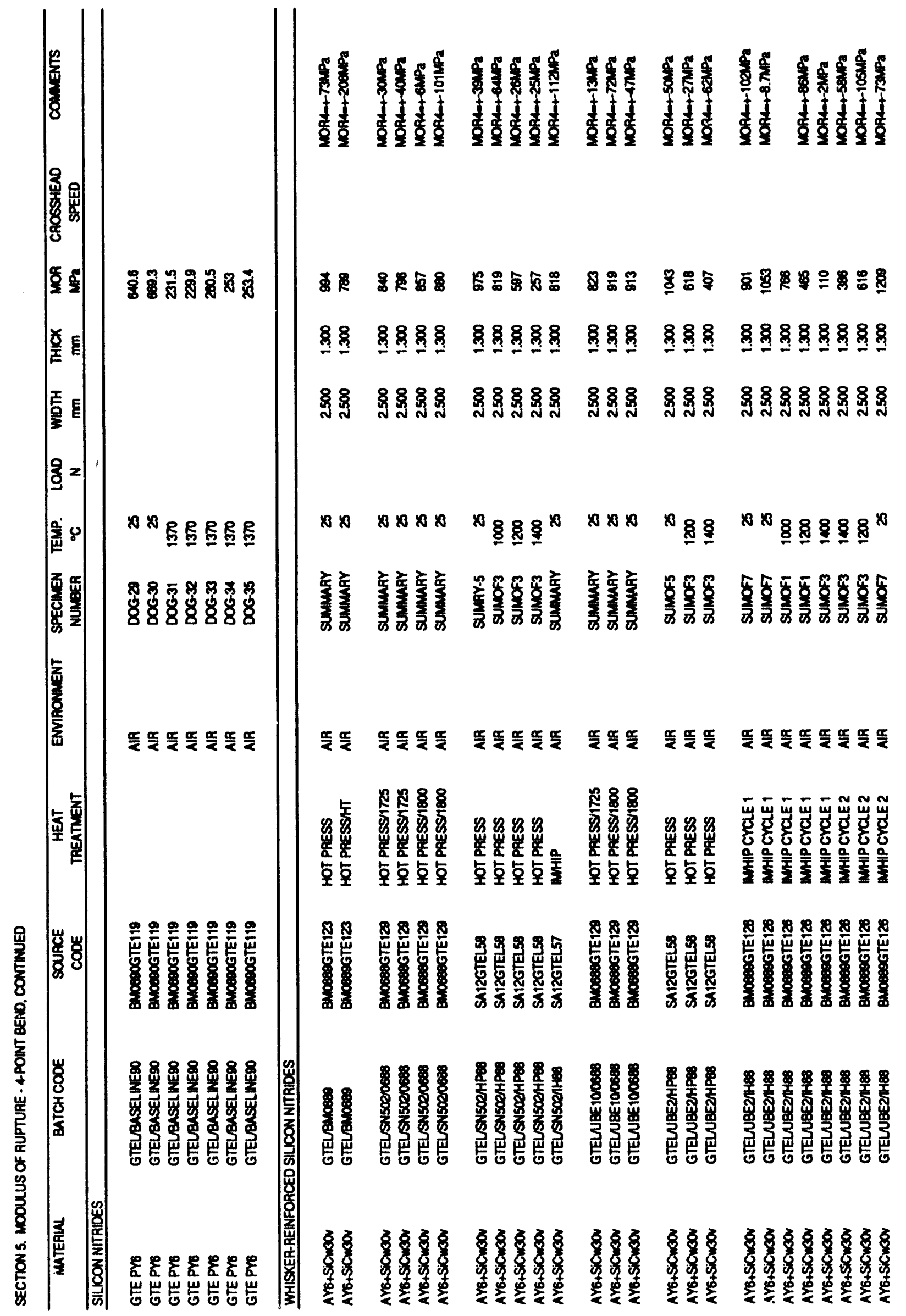




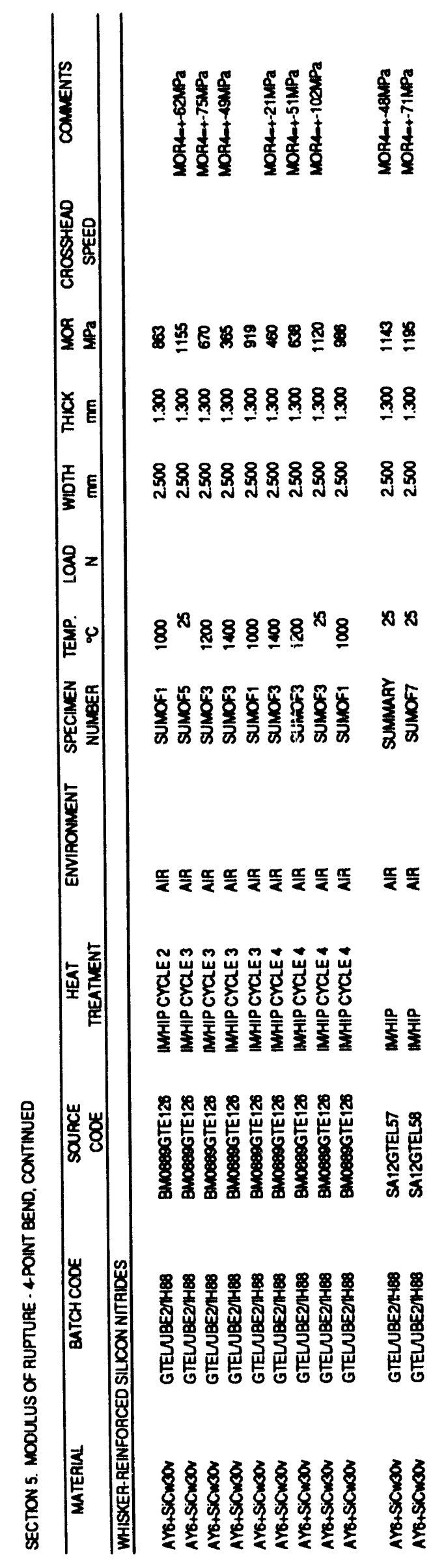




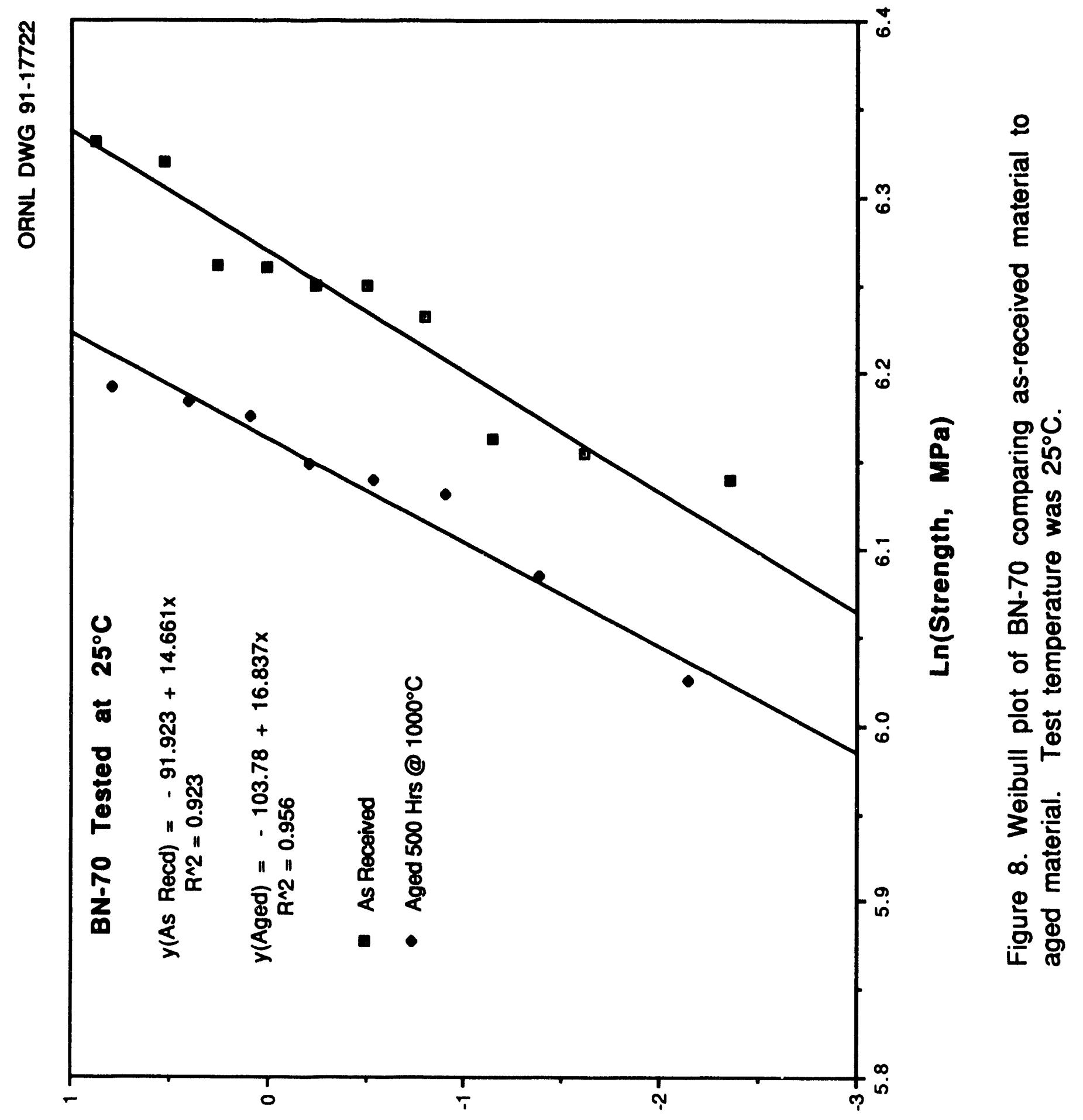

$[(\exists-1) / L] \cup 747$ 


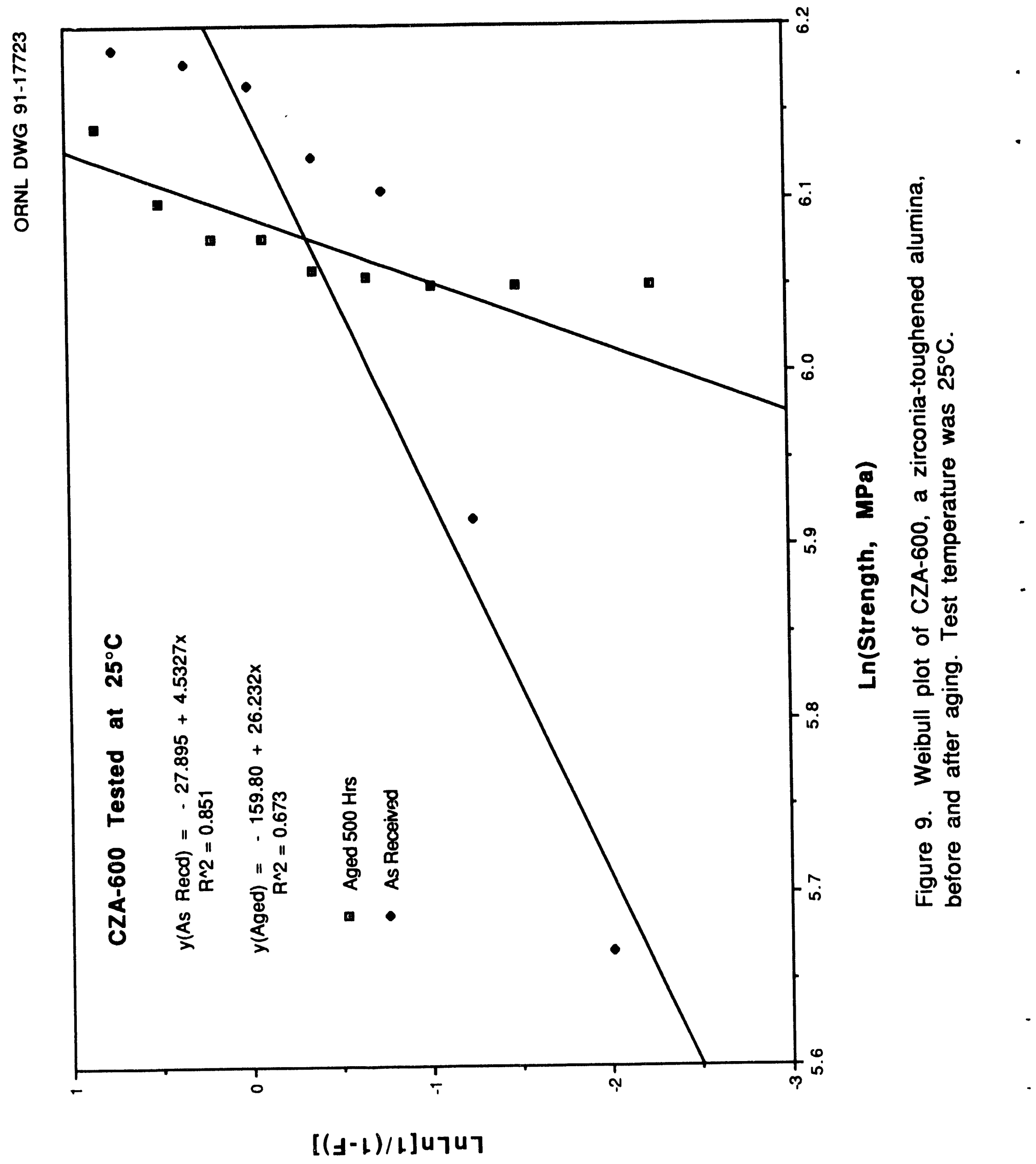




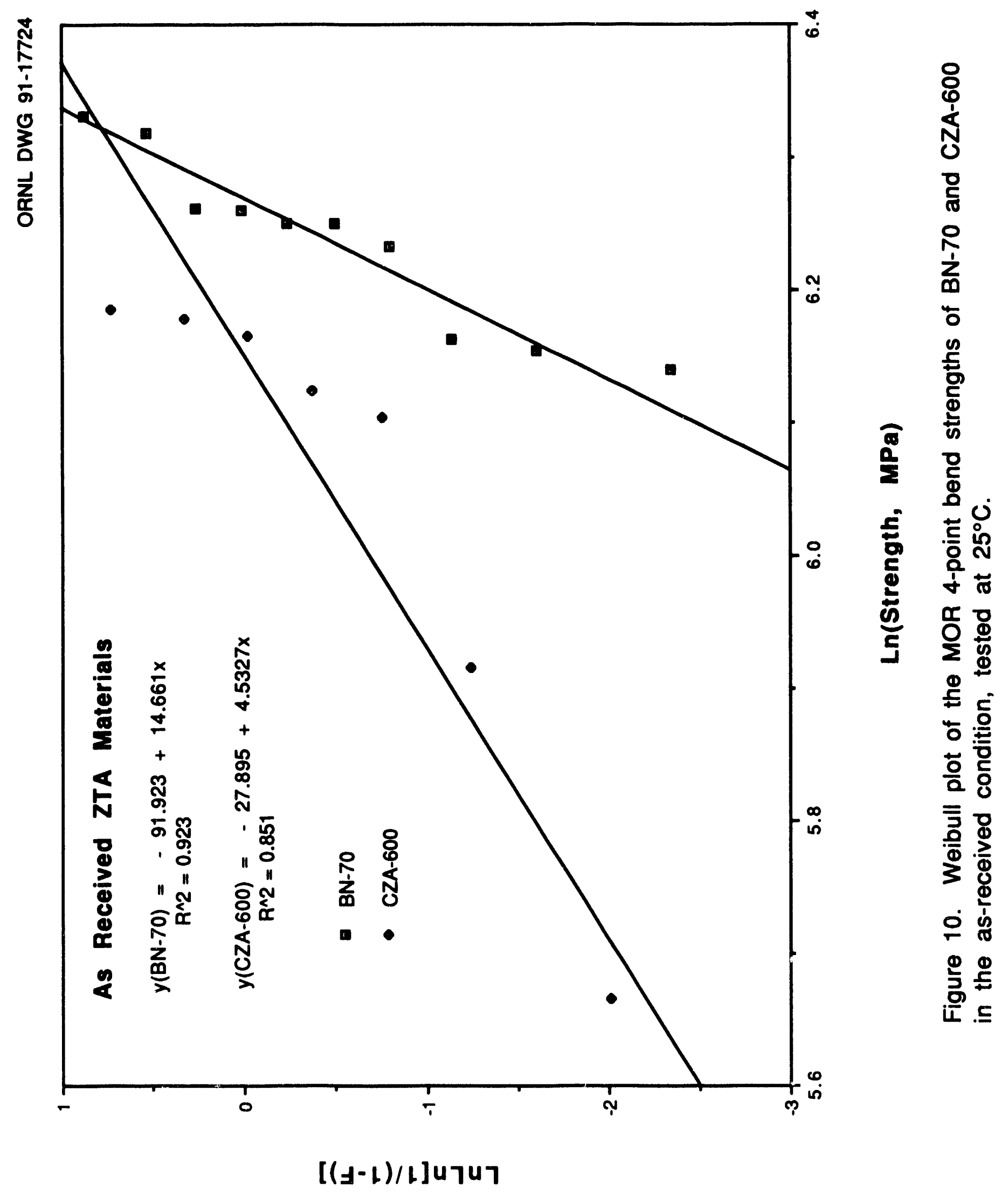




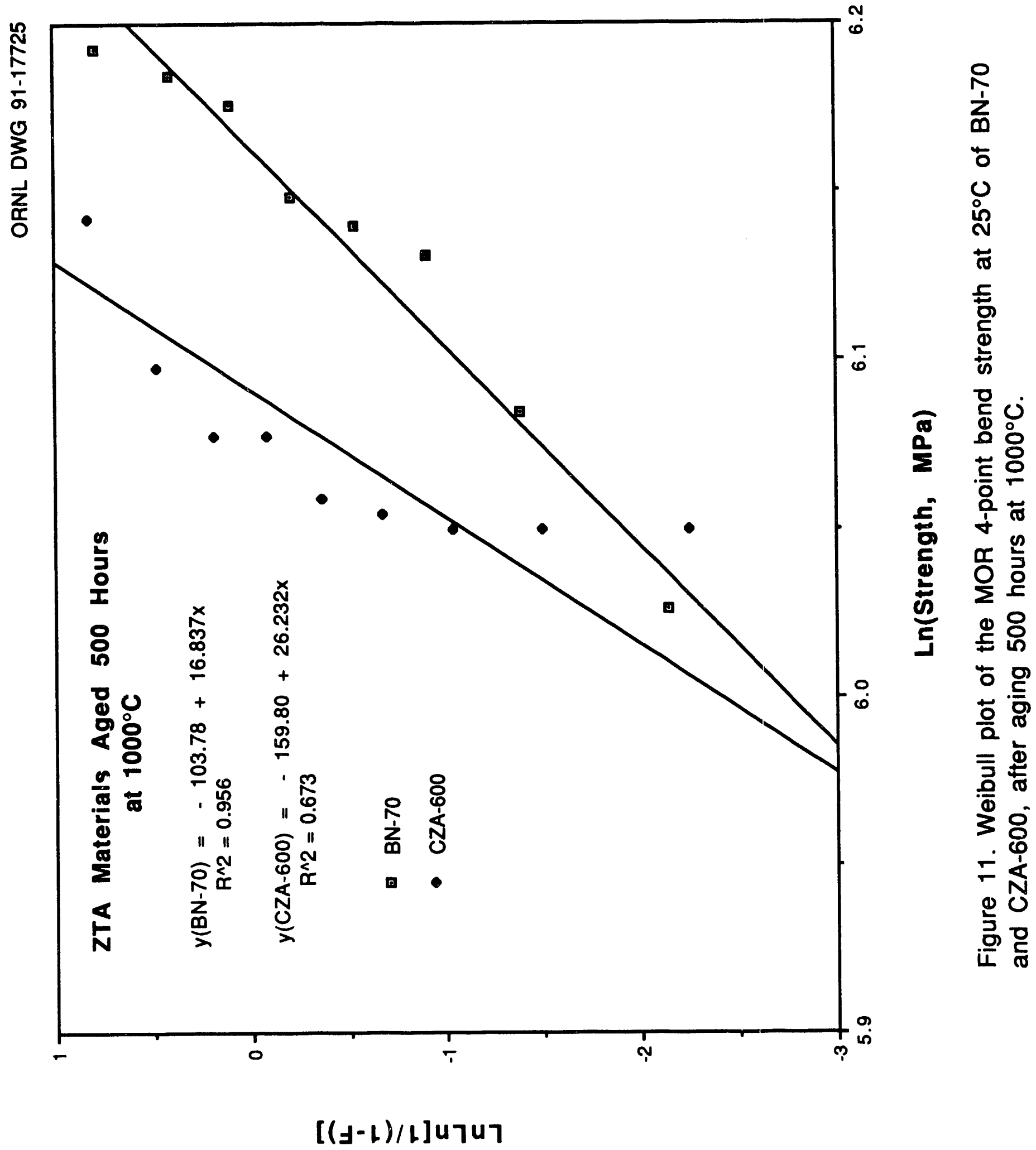




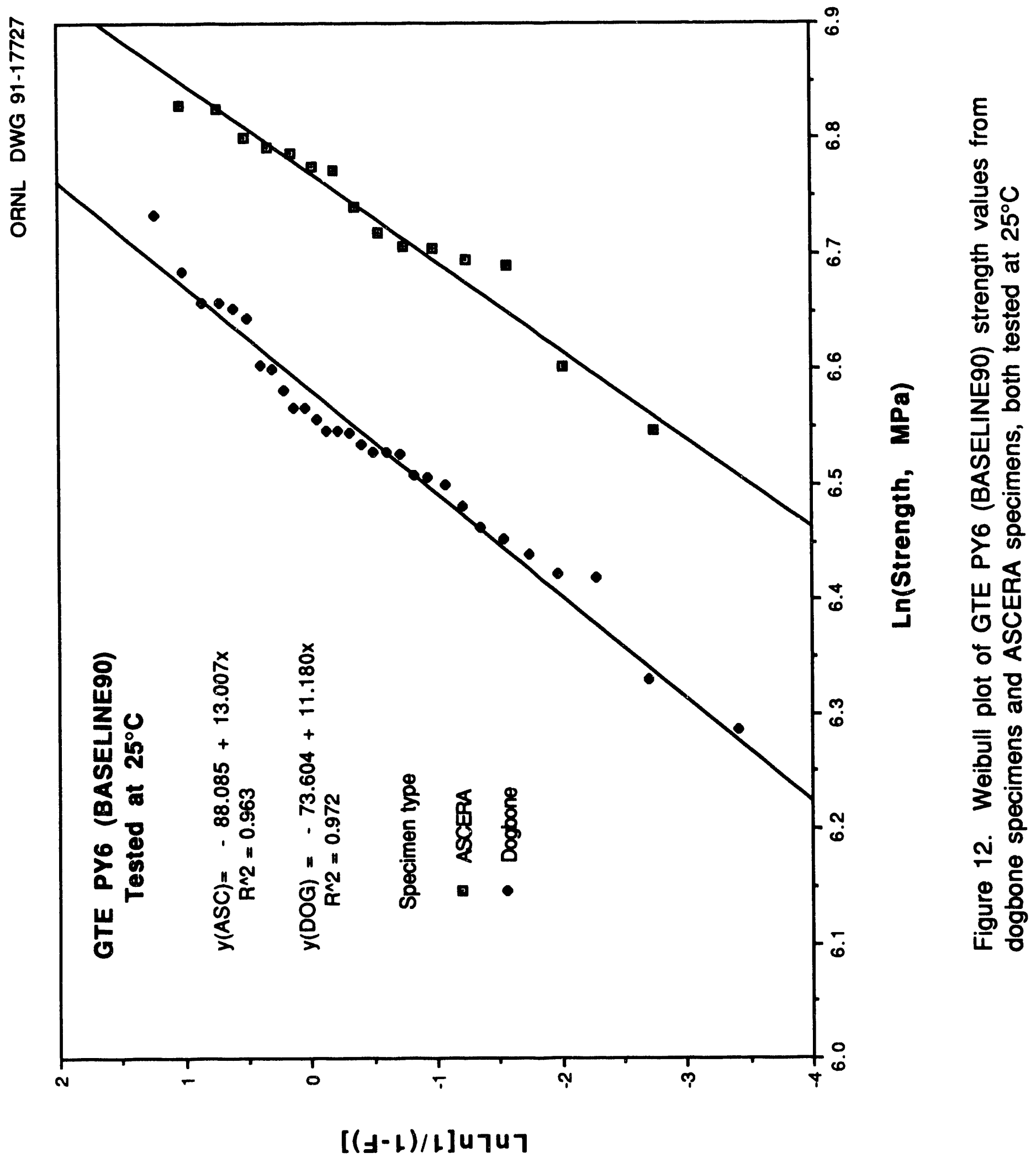




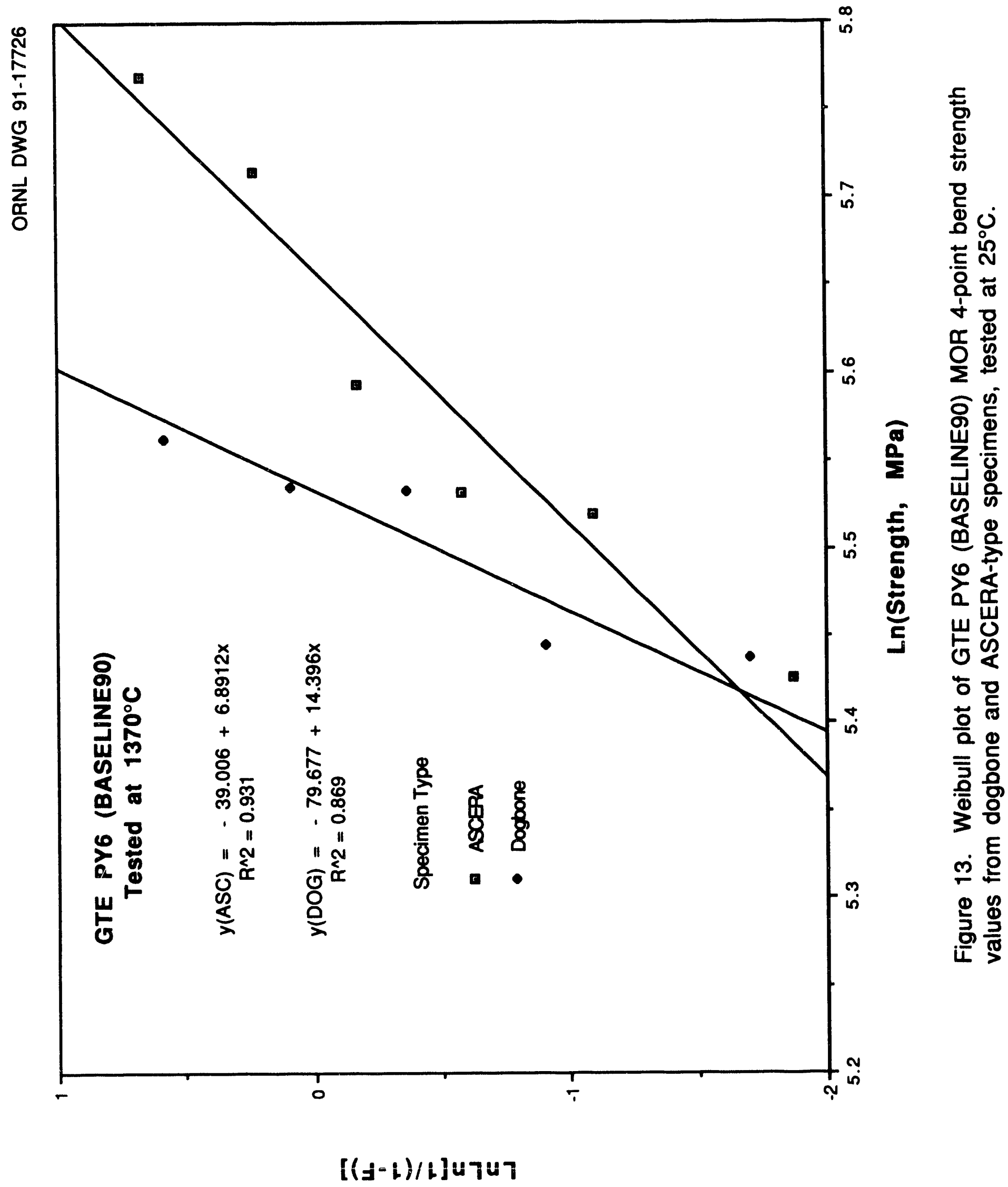




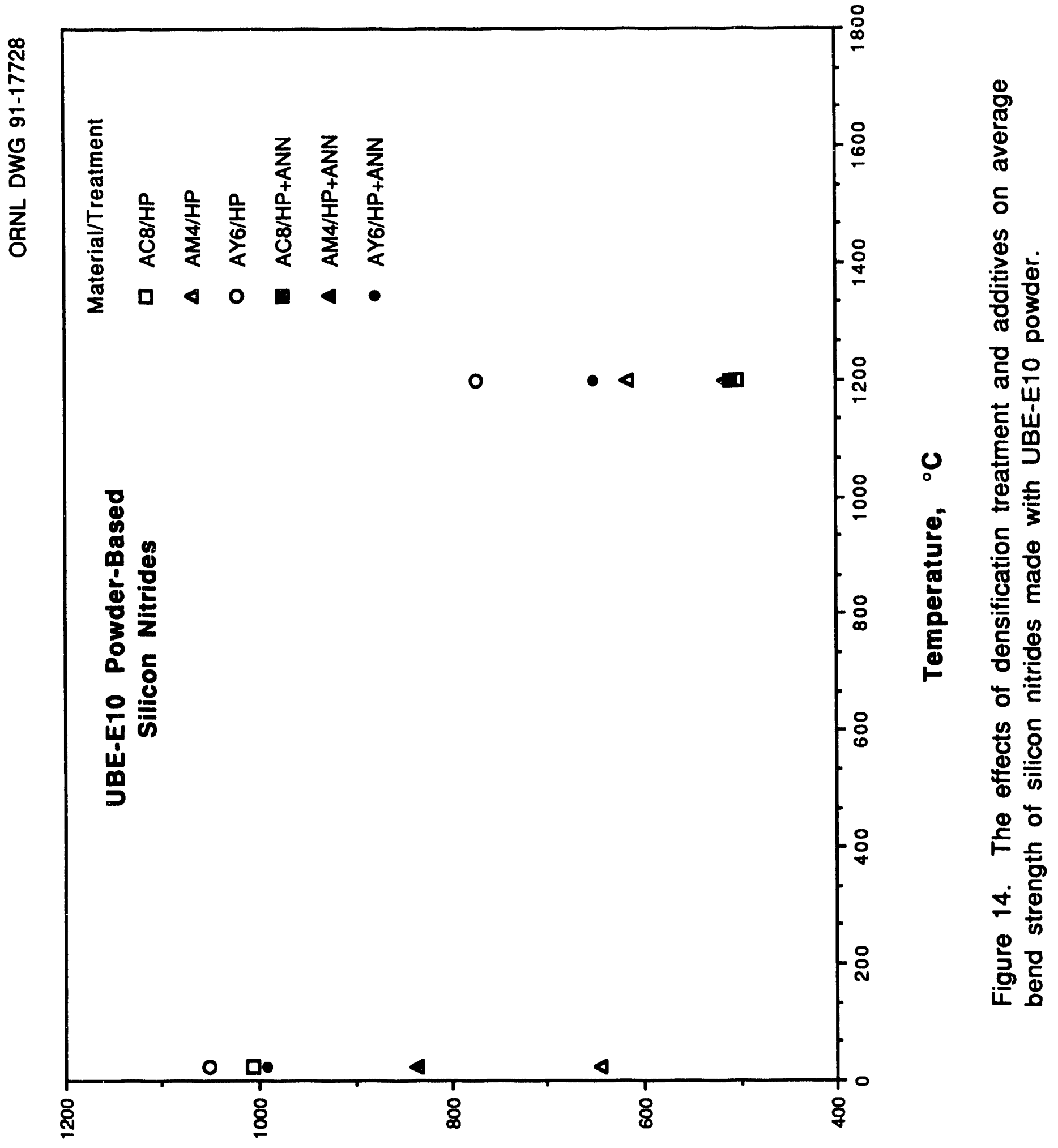

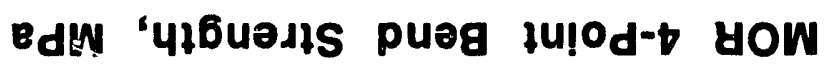




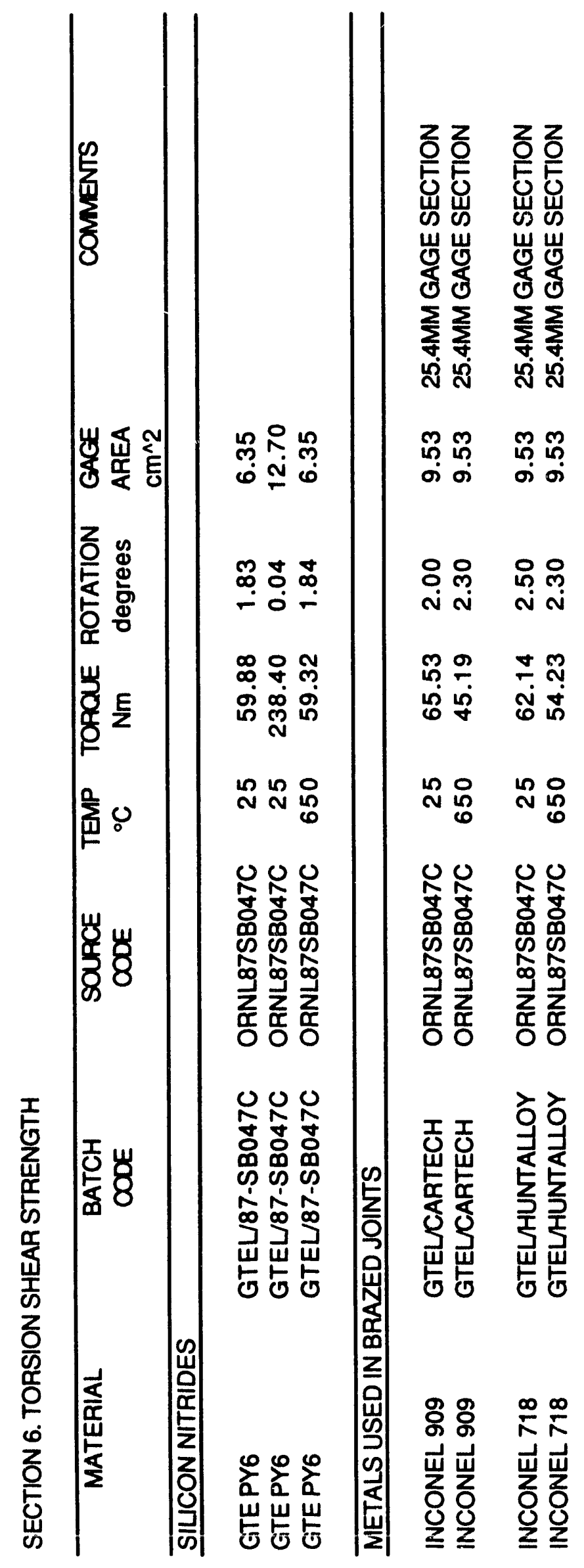


SECTION 7. MODULUS OF RUPTURE- 4 POINT BEND OF BRAZED SPECIMENS

MATERLAL 1 = SN-220 coated with titanium, BATCH CODE = KYOCERAMS890

MATERIAL 2 = SN-220 coated with thanlum, BATCH CODE = KYOCERAMS890

BRAZE = AU-25Ni-25Pd

SOURCE CODE = SANTELLABPO

JOINT CODE = ORN MSEOSINAT

\begin{tabular}{|c|c|c|c|c|c|c|c|c|}
\hline $\begin{array}{c}\text { SPECIMEN } \\
\text { NUMBER }\end{array}$ & $\begin{array}{l}\text { BRAZING } \\
\text { TREATMENT }\end{array}$ & $\begin{array}{l}\text { SPECIMEN } \\
\text { CONDITION }\end{array}$ & ENV. & $\begin{array}{c}\text { TEMP } \\
{ }^{\circ} \mathrm{C} \\
\end{array}$ & $\begin{array}{l}\text { MOR } \\
\mathrm{MPa}\end{array}$ & $\begin{array}{c}\text { LOAD } \\
\mathrm{N} \\
\end{array}$ & $\begin{array}{c}\text { WIDTH } \\
\mathrm{mm}\end{array}$ & $\begin{array}{c}\text { THICK } \\
\mathrm{mm} \\
\end{array}$ \\
\hline 1 & BRAZED IN VAC@1030C & AS BRAZIED & AlR & 25 & 441.3 & & 2.5 & 2.0 \\
\hline 2 & BRAZED IN VAC@ $1030 C$ & AS BRAZIED & AIR & 25 & 419.4 & & 2.5 & 2.0 \\
\hline 3 & BRAZED IN VAC@1030C & AS BRAZIED & AlR & 25 & 457.5 & & 2.5 & 2.0 \\
\hline 4 & BRAZED IN VACQ1030C & AS BRAZED & AIR & 25 & 414.9 & & 2.5 & 2.0 \\
\hline 5 & BRAZED IN VAC@1030C & AS BRAZIED & AIR & 25 & 444.8 & & 2.5 & 2.0 \\
\hline 6 & BRAZED IN VAC@1030C & AS BRAZIED & AIR & 25 & 387.1 & & 2.5 & 2.0 \\
\hline 7 & BRAZED IN VAC@1030C & AS BRAZIED & AIR & 25 & 385.6 & & 2.5 & 2.0 \\
\hline 8 & BRAZED IN VAC@1030C & AS BRAZZD & AIR & 25 & 373.9 & & 2.5 & 2.0 \\
\hline 8 & BRAZED IN VAC@1030C & AS BRAZED & AIR & 25 & 438.3 & & 2.5 & 2.0 \\
\hline 10 & BRAZED IN VAC@1030C & AS BRAZIED & AIR & 25 & 347.4 & & 2.5 & 2.0 \\
\hline 11 & BRAZED IN VAC@1030C & AS BRAZIED & AIR & 25 & 340.3 & & 2.5 & 2.0 \\
\hline 12 & BRAZED IN VAC@1030C & AS BRAZIED & AlR & 25 & 352.7 & & 2.5 & 2.0 \\
\hline 13 & BRAZED IN VAC@1030C & AS BRAZIED & AlR & 25 & 344.8 & & 2.5 & 2.0 \\
\hline 14 & BRAZED IN VAC@1030C & AS BRAZZD & AIR & 25 & 495.8 & & 2.5 & 2.0 \\
\hline 15 & BRAZED IN VAC@1030C & AS BRAZED & AIR & 25 & 414.9 & & 2.5 & 2.0 \\
\hline 16 & BRAZED IN VAC@1030C & AS BRAZIED & AIR & 25 & 465.2 & & 2.5 & 2.0 \\
\hline 17 & BRAZED IN VAC@1030C & AS BRAZZD & AIR & 25 & 474.9 & & 2.5 & 2.0 \\
\hline 18 & BRAZED IN VAC@1030C & AS BRAZIED & AIR & 25 & 292.8 & & 2.5 & 2.0 \\
\hline MCB-451A & BRAZED IN VAC@1030C & AS BRAZED & AIR & 600 & 381.2 & 382.7 & 3.1064 & 2.4714 \\
\hline MCB-451B & BRAZED IN VAC@1030C & AS BRAZED & AIR & 600 & 392.3 & 391.5 & 3.1064 & 2.4790 \\
\hline MCB-451C & BFWZED IN VAC@1030C & AS BRAZZD & AIR & 600 & 513.3 & 515.6 & 3.0607 & 2.4892 \\
\hline MCB-451D & BRAZED IN VAC@1030C & AS BRAZIED & AIR & 600 & 360.7 & 360.0 & 3.0937 & 2.4841 \\
\hline MCB-451E & BRAZED IN VAC@1030C & AS BRAZZED & AlR & 600 & 367.9 & 364.1 & 3.1181 & 2.4841 \\
\hline MCB-451F & BRAZED IN VAC@1030C & AS BRAZED & AIR & 600 & 375.9 & 381.4 & 3.0734 & $2.4714^{\circ}$ \\
\hline MCB-452A & BRAZED IN VAC@1030C & AS BRAZED & AIR & 600 & 361.2 & 362.1 & 3.0734 & 2.4867 \\
\hline MCB-452B & BRAZED IN VAC@1030C & AS BRAZED & AIR & 600 & 448.4 & 446.7 & 3.0988 & 2.4841 \\
\hline MCB-452C & BRAZED IN VAC@1030C & AS BRAZED & AIR & 600 & 386.5 & 386.7 & 3.0988 & 2.4790 \\
\hline MCB-452D & BRAZED IN VAC@1030C & AS BRAZED & AIR & 600 & 440.4 & 416.4 & 3.2715 & 2.4816 \\
\hline MCB-452E & BRAZED IN VAC@1030C & AS BRAZED & AIR & 600 & 419.5 & 420.0 & 3.0861 & 2.4816 \\
\hline MCB-452F & BRAZED IN VAC@1030C & AS BRAZZD & AlR & 600 & 382.1 & 385.5 & 3.0861 & 2.4765 \\
\hline MCB-453A & BRAZED IN VAC@1030C & AS BRAZIED & AIR & 600 & 412.8 & 415.8 & 3.0963 & 2.4714 \\
\hline MCB-453B & BRAZED IN VAC@1030C & AS BRAZZD & AIR & 600 & 255.3 & 259.5 & 3.0683 & 2.4714 \\
\hline MCB-453C & BRAZED IN VAC@1030C & AS BRAZZD & AIR & 600 & 334.9 & 336.3 & 3.1064 & 2.4714 \\
\hline MCB-453D & BRAZED IN VAC@1030C & AS BRAZED & AIR & 600 & 312.3 & 310.3 & 3.1191 & 2.4790 \\
\hline MCB-453E & BRAZED IN VAC@1030C & AS BRAZIED & AIR & 600 & 316.3 & 318.8 & 3.0810 & 2.4765 \\
\hline MCB- $453 F$ & BRAZED IN VAC@1030C & AS BRAZED & AIR & 600 & 318.9 & 320.2 & 3.1064 & 2.4714 \\
\hline
\end{tabular}


SECTION 7. MODULUS OF RUPTURE- 4 POINT BEND OF BRAZED SPECIMENS, CONTINUED

MATERIAL 1 = SN 220 coatod with titanium, BATCH CODE = KYOCERAMS890

MATERIAL 2 = SN-220 coated with tianium, BATCH CODE = KYOCERAMS890

BRAZE $=$ 50AU-25Nt-25Pd

SOURCE CODE $=$ SANTELLAB9O

JOINT CODE = ORNUMS890/SNG1

\begin{tabular}{|c|c|c|c|c|c|c|c|}
\hline $\begin{array}{c}\text { SPECIMEN } \\
\text { NUMBER }\end{array}$ & $\begin{array}{l}\text { BRAZING } \\
\text { TREATMENT }\end{array}$ & $\begin{array}{l}\text { SPECIMEN } \\
\text { CONDITION }\end{array}$ & ENN. & $\begin{array}{c}\text { TEMP } \\
{ }^{\circ} \mathrm{C} \\
\end{array}$ & $\begin{array}{l}\text { MOR } \\
\mathrm{MPa} \\
\end{array}$ & $\begin{array}{c}\text { WIDTH } \\
\mathrm{mm}\end{array}$ & $\begin{array}{c}\text { THICK } \\
\mathrm{mm} \\
\end{array}$ \\
\hline 1 & BRAZED IN VAC@1030C & AS BRAZEDD & AIR & 25 & 428.2 & 2.5 & 2.0 \\
\hline 2 & BRAZED IN VAC@1030C & AS BRAZZD & AIR & 25 & 404.8 & 2.5 & 2.0 \\
\hline 3 & BRAZED IN VAC@1C3OC & AS BRAZED & AlR & 25 & 421.9 & 2.5 & 2.0 \\
\hline 4 & BRAZED IN VAC@1030C & AS BRAZED & AIR & 25 & 209.3 & 2.5 & 2.0 \\
\hline 5 & BRAZED IN VAC@1030C & AS BRAZED & AlR & 25 & 345.2 & 2.5 & 2.0 \\
\hline 6 & BRAZED IN VAC@1030C & AS BRAZED & AIR & 25 & 404.8 & 2.5 & 2.0 \\
\hline 7 & BRAZED IN VAC@1030C & AS BRAZED & AIR & 25 & 412.7 & 2.5 & 2.0 \\
\hline 8 & BRAZED IN VAC@1030C & AS BRAZZD & AIR & 25 & 444.6 & 2.5 & 2.0 \\
\hline 9 & BRAZED IN VAC@1030C & AS BRAZZD & AIR & 25 & 394.7 & 2.5 & 2.0 \\
\hline 10 & BRAZED IN VAC@1030C & AS BRAZIED & AIR & 25 & 425.8 & 2.5 & 2.0 \\
\hline 11 & BRAZED IN VAC@1030C & AS BRAZZD & AIR & 25 & 464.6 & 2.5 & 2.0 \\
\hline 12 & BRAZED IN VAC@1030C & AS BRAZZD & AIR & 25 & 453.7 & 2.5 & 2.0 \\
\hline 13 & BRAZED IN VAC@1030C & AS BRAZZD & AlR & 25 & 378.2 & 2.5 & 2.0 \\
\hline 14 & BRAZED IN VAC@1030C & AS BRAZED & AlR & 25 & 442.7 & 2.5 & 2.0 \\
\hline 15 & BRAZED IN VAC@1030C & AS BRAZED & AlR & 25 & 425.0 & 2.5 & 2.0 \\
\hline
\end{tabular}

MATERIAL $1=$ SN-220 coated with titanium, BATCH CODE = KYOCERAMS890

MATERIAL $2=$ SN-220 coated with titanium, BATCH CODE $=$ KYOCERAMS890

BRAZE $=50 \mathrm{Au}-25 \mathrm{Ni}-25 \mathrm{Pd}$

SOURCE CODE = SANTELLAB9O

JOINT CODE = ORNLMSB9O/SNG2

\begin{tabular}{|c|c|c|c|c|c|c|c|c|}
\hline $\begin{array}{c}\text { SPECIMEN } \\
\text { NUMBER }\end{array}$ & $\begin{array}{c}\text { BRAZING } \\
\text { TREATMENT }\end{array}$ & $\begin{array}{l}\text { SPECIMEN } \\
\text { CONDIIION } \\
\end{array}$ & ENV. & $\begin{array}{c}\text { TEMP } \\
{ }^{\circ} \mathrm{C} \\
\end{array}$ & $\begin{array}{l}\text { MOR } \\
\mathrm{MPa} \\
\end{array}$ & $\begin{array}{c}\text { LOAD } \\
\mathrm{N} \\
\end{array}$ & $\begin{array}{c}\text { WIDTH } \\
\mathrm{mm}\end{array}$ & $\begin{array}{c}\text { THICK } \\
\mathrm{mm}\end{array}$ \\
\hline MCB-444A & BRAZED IN VAC@1030C & AS BRAZED & AlR & 600 & 336.7 & 330.7 & 3.099 & 2.502 \\
\hline MCB-444B & BRAZED IN VAC@1030C & AS BRAZED & AlR & 600 & 444.8 & 442.4 & 3.078 & 2.494 \\
\hline MCB-444C & BRAZED IN VAC@1030C & AS BRAZED & AlR & 600 & 390.5 & 382.8 & 3.099 & 2.504 \\
\hline MCB-444D & BRAZED IN VAC@1030C & AS BRAZED & AlR & 600 & 390.5 & 386.0 & 3.073 & 2.504 \\
\hline MCB-444E & BRAZED IN VAC@1030C & AS BRAZED & AlR & 600 & 289.6 & 292.1 & 3.061 & 2.484 \\
\hline MCB-444F & BRAZED IN VAC@1030C & AS BRAZED & AIR & 600 & 426.6 & 420.8 & 3.104 & 2.494 \\
\hline MCB-445A & BRAZED IN VAC@1030C & AS BRAZIED & AlR & 600 & 401.2 & 400.4 & 3.068 & 2.494 \\
\hline MCB-445B & BRAZED IN VAC@1030C & AS BRAZED & AlR & 600 & 448.4 & 442.1 & 3.112 & 2.492 \\
\hline MCB-445C & BRAZED IN VAC@1030C & AS BRAZED & AlR & 600 & 440.4 & 436.4 & 3.096 & 2.492 \\
\hline MCB-445D & BRAZED IN VAC@1030C & AS BRAZED & AlR & 600 & 386.5 & 388.1 & 3.068 & 2.487 \\
\hline MCB-445E & BRAZED IN VAC@1030C & AS BRAZED & AlR & 600 & 203.3 & 204.9 & 3.081 & 2.477 \\
\hline MCB-445F & BRAZED INVAC@1030C & AS BRAZED & AlR & 600 & 390.5 & 389.4 & 3.096 & 2.484 \\
\hline MCB-446A & BRAZED IN VAC@1030C & AS BRAZED & AlR & 600 & 433.2 & 440.6 & 3.086 & 2.464 \\
\hline MCB-446B & BRAZED IN VAC@1030C & AS BRAZED & AlR & 600 & 458.6 & 468.1 & 3.043 & 2.477 \\
\hline MCB-446C & BRAZED IN VAC@1030C & AS BRAZED & AIR & 600 & 446.6 & 459.8 & 3.086 & 2.449 \\
\hline MCB-446D & BRAZED INVAC@1030C & AS BRAZED & AlR & 600 & 335.8 & 340.1 & 3.112 & 2.459 \\
\hline MCB-446E & BRAZED IN VAC@1030C & AS BRAZED & AlR & 600 & 473.7 & 486.8 & 3.112 & 2.441 \\
\hline $\mathrm{MCB}-446 \mathrm{~F}$ & BRAZED IN VAC@1030C & AS BRAZED & AlR & 600 & 414.6 & 394.9 & 3.343 & 2.446 \\
\hline
\end{tabular}


SECTION 7. MODULUS OF RUPTURE- 4 POINT BEND OF BRAZED SPECIMENS, CONTINUED

MATERLAL 1 = MS-PSZ coated with thanlum, BATCH CODE = NLSENORNUJ1

MATERIAL 2 = NODULAR CAST IRON, BATCH CODE = CUMMINS GRADE 8003

BRAZE = Ag-30Cu-10Sn

SOURCE CODE = SANTELLAQPOO

JOINT CODE = ORM MMSESORZRFE 1

\begin{tabular}{|c|c|c|c|c|c|c|c|c|}
\hline $\begin{array}{c}\text { SPECIMEN } \\
\text { NUMBER }\end{array}$ & $\begin{array}{l}\text { BRAZING } \\
\text { TREATMENT }\end{array}$ & $\begin{array}{l}\text { SPECIMEN } \\
\text { CONDITION }\end{array}$ & ENV. & $\begin{array}{c}\text { TEMP } \\
{ }^{\circ} \mathrm{C} \\
\end{array}$ & $\begin{array}{l}\text { MOR } \\
\text { MPa }\end{array}$ & $\begin{array}{c}\text { WIDTH } \\
\mathrm{mm}\end{array}$ & $\begin{array}{c}\text { THICK } \\
\mathrm{mm} \\
\end{array}$ & $\begin{array}{c}\text { LOAD RATE } \\
\mathrm{mm} / \mathrm{sec}\end{array}$ \\
\hline MCB-465C & BRAZED N VAC@735C & AS BRAZZD & AIR & 25 & 91.7 & 2.50 & 2.00 & 22.70 \\
\hline MCB-461A & BRAZED IN VACQ735C & AS BRAZIED & AIR & 25 & 125.3 & 2.50 & 2.00 & 22.70 \\
\hline MCB-466C & BRAZED IN VAC@735C & AS BRAZIED & AIR & 25 & 129.2 & 2.50 & 2.00 & 22.70 \\
\hline MCB-4550 & BRAZED IN VAC@735C & AS BRAZIED & AIR & 25 & 180.7 & 2.50 & 2.00 & 22.70 \\
\hline MCB-4650 & BRAZED W VAC@735C & AS BRAZED & AIR & 25 & 208.7 & 2.50 & 2.00 & 22.70 \\
\hline MCB-459A & BRAZED IN VAC@735C & AS BRAZED & AIR & 25 & 238.4 & 2.50 & 2.00 & 22.70 \\
\hline MCB-455B & BRAZED IN VAC@735C & AS BRAZIED & AIR & 25 & 299.7 & 2.50 & 2.00 & 22.70 \\
\hline MCB-465B & BRAZED IN VAC@735C & AS BRAZED & AIR & 25 & 304.8 & 2.50 & 2.00 & 22.70 \\
\hline MCB-458A & BRAZED IN VAC@735C & AS BRAZZD & AIR & 25 & 306.9 & 2.50 & 2.00 & 22.70 \\
\hline MCB-455E & BRAZED IN VACQ735C & AS BRAZIED & AIR & 25 & 312.6 & 2.50 & 2.00 & 22.70 \\
\hline MCB-463B & BRAZED IN VAC@735C & AS BRAZIED & AlR & 25 & 317.8 & 2.50 & 2.00 & 22.70 \\
\hline MCB-4668 & BRAZED IN VAC@735C & AS BRAZED & AIR & 25 & 320.8 & 2.50 & 2.00 & 22.70 \\
\hline MCB-455A & BRAZED IN VAC@735C & AS BRAZED & AIR & 25 & 323.2 & 2.50 & 2.00 & 22.70 \\
\hline MCB-466A & BRAZED IN VAC@735C & AS BRAZZD & AIR & 25 & 323.7 & 2.50 & 2.00 & 22.70 \\
\hline MCB-465A & BRAZED IN VAC@735C & AS BRAZED & AIR & 25 & 327.5 & 2.50 & 2.00 & 22.70 \\
\hline MCB-455F & BRAZED IN VAC@735C & AS BRAZZD & AlR & 25 & 332.2 & 2.50 & 2.00 & 22.70 \\
\hline MCB-465E & BRAZED IN VAC@735C & AS BRAZED & AIR & 25 & 337.8 & 2.50 & 2.00 & 22.70 \\
\hline MCB-463C & BRAZED IN VAC@735C & AS BRAZED & AIR & 25 & 350.4 & 2.50 & 2.00 & 22.70 \\
\hline MCB-455C & BRAZED IN VAC@735C & AS BRAZZD & AlR & 25 & 370.3 & 2.50 & 2.00 & 22.70 \\
\hline MCB-456A & BRAZED IN VAC@735C & AS BRAZIED & AIR & 25 & 375.2 & 2.50 & 2.00 & 22.70 \\
\hline MCB-463A & DRAZED IN VAC@735C & AS BAAZED & AIR & 25 & 377.0 & 2.50 & 2.00 & 22.70 \\
\hline MCB-463D & BRAZED IN VAC@735C & AS BRAZZD & AIR & 25 & 378.0 & 2.50 & 2.00 & 22.70 \\
\hline
\end{tabular}

MATERLAL 1 = MS-PSZ coated with titanium, BATCH CODE $=$ NLSENORNLJ1

MATERIAL 2 = NODULAR CAST IRON, BATCH CODE = CUMMINS GRADE 8003

BRAZE $=A g-30 \mathrm{Cu}-10 \mathrm{Sn}$

SOURCE CODE = SANTELLABPO

JOINT CODE = ORNUMSBSORRFE2

\begin{tabular}{ccccccccc}
\hline $\begin{array}{c}\text { SPECIMEN } \\
\text { NUMBER }\end{array}$ & $\begin{array}{c}\text { BRAZING } \\
\text { TREATMENT }\end{array}$ & $\begin{array}{c}\text { SPECIMEN } \\
\text { CONDITION }\end{array}$ & $\begin{array}{c}\text { ENV. } \\
\text { MCB-462A }\end{array}$ & $\begin{array}{c}\text { BEMPAZED IN VAC@735C } \\
{ }^{\circ} \mathrm{C}\end{array}$ & $\begin{array}{c}\text { MOR } \\
\text { MPa }\end{array}$ & $\begin{array}{c}\text { WIDTH } \\
\mathrm{mm}\end{array}$ & $\begin{array}{c}\text { THICK } \\
\mathrm{mm}\end{array}$ & $\begin{array}{c}\text { LOAD RATE } \\
\mathrm{mm} / \mathrm{seC}\end{array}$ \\
\hline MCB-456B & BRAZED IN VAC@735C & AIR & 400 & 109.3 & 2.50 & 2.00 & 22.70 \\
MCB-460D & BRAZED IN VAC@735C & AS BRAZED & AIR & 400 & 117.5 & 2.50 & 2.00 & 22.70 \\
MCB-462D & BRAZED IN VAC@735C & AS BRAZED & AIR & 400 & 130.7 & 2.50 & 2.00 & 22.70 \\
MCB-460A & BRAZED IN VAC@735C & AS BRAZED & AIR & 400 & 143.0 & 2.50 & 2.00 & 22.70 \\
MCB-462C & BRAZED IN VAC@735C & AS BRAZED & AIR & 400 & 146.2 & 2.50 & 2.00 & 22.70 \\
MCB-460B & BRAZED IN VAC@735C & AS BRAZED & AIR & 400 & 150.3 & 2.50 & 2.00 & 22.70 \\
MCB-462B & BRAZED IN VAC@735C & AS BRAZED & AIR & 400 & 177.2 & 2.50 & 2.00 & 22.70 \\
MCB-460E & BRAZED IN VAC@735C & AS BRAZED & AIR & 400 & 188.8 & 2.50 & 2.00 & 22.70 \\
MCB-462E & BRAZED IN VAC@735C & AS BRAZED & AIR & 400 & 191.2 & 2.50 & 2.00 & 22.70 \\
MCB-460C & BRAZED IN VAC@735C & AS BRAZED & AIR & 400 & 195.7 & 2.50 & 2.00 & 22.70 \\
MCB-456E & BRAZED IN VAC@735C & AS BRAZED & AIR & 400 & 213.2 & 2.50 & 2.00 & 22.70 \\
MCB-456D & BRAZED IN VAC@735C & AS BRAZED & AIR & 400 & 220.6 & 2.50 & 2.00 & 22.70 \\
MCB-456C & BRAZED IN VAC@735C & AS BRAZED & AIR & 400 & 227.1 & 2.50 & 2.00 & 22.70 \\
MCB-458B & BRAZED IN VAC@735C & AS BRAZED & AIR & 400 & 243.3 & 2.50 & 2.00 & 22.70
\end{tabular}




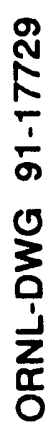

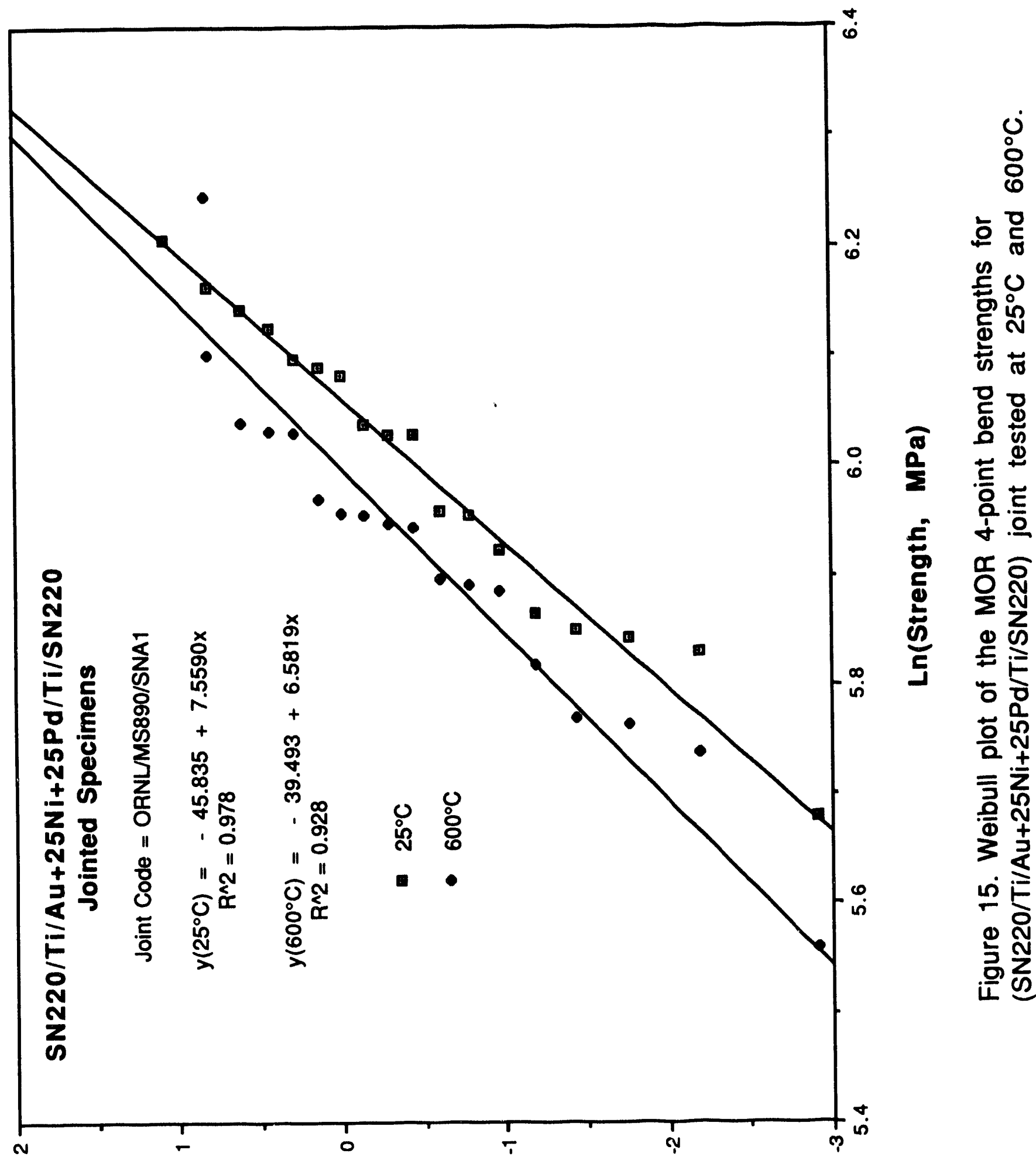

$[(y-L\} / L] \cup 747$ 


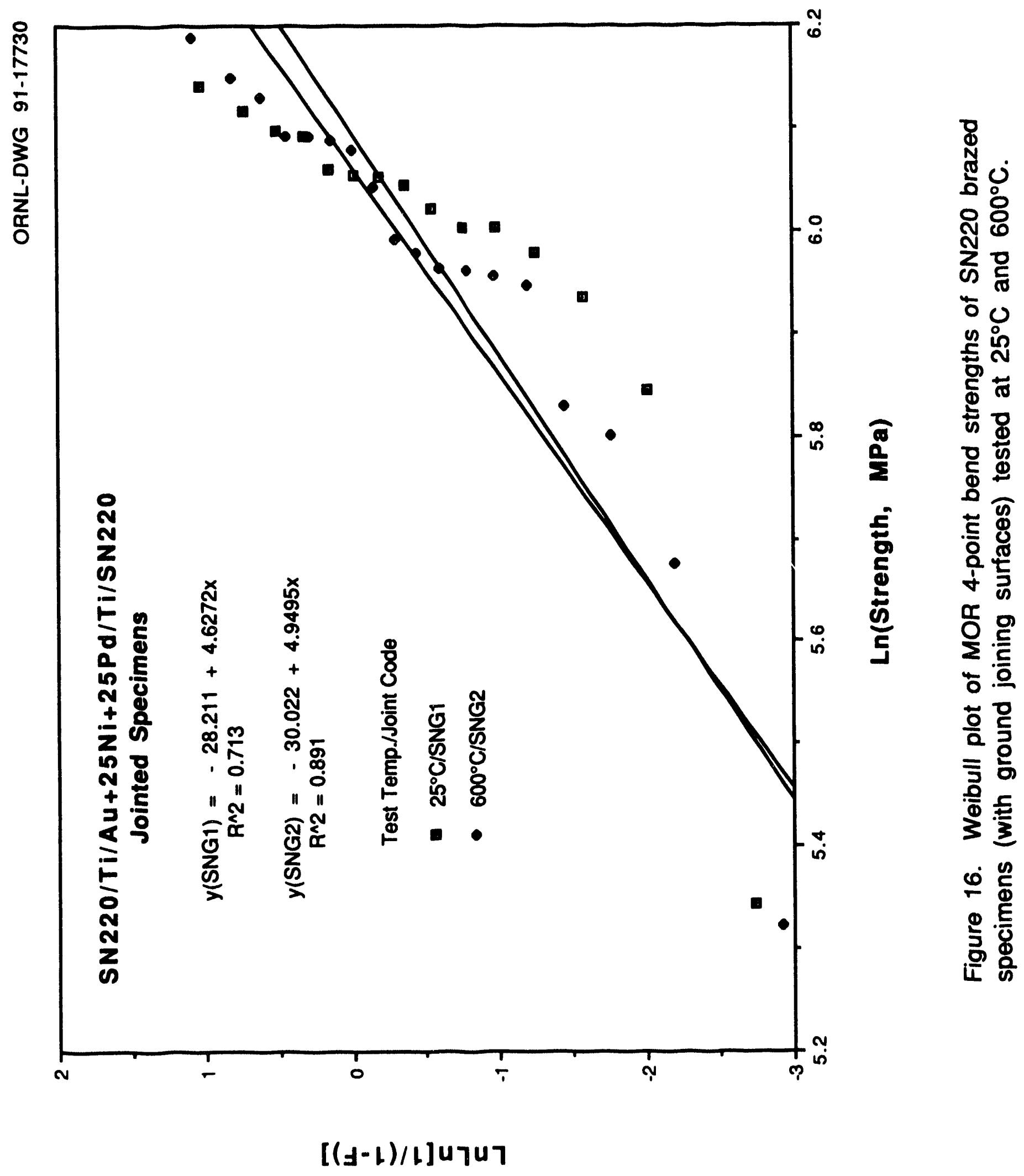




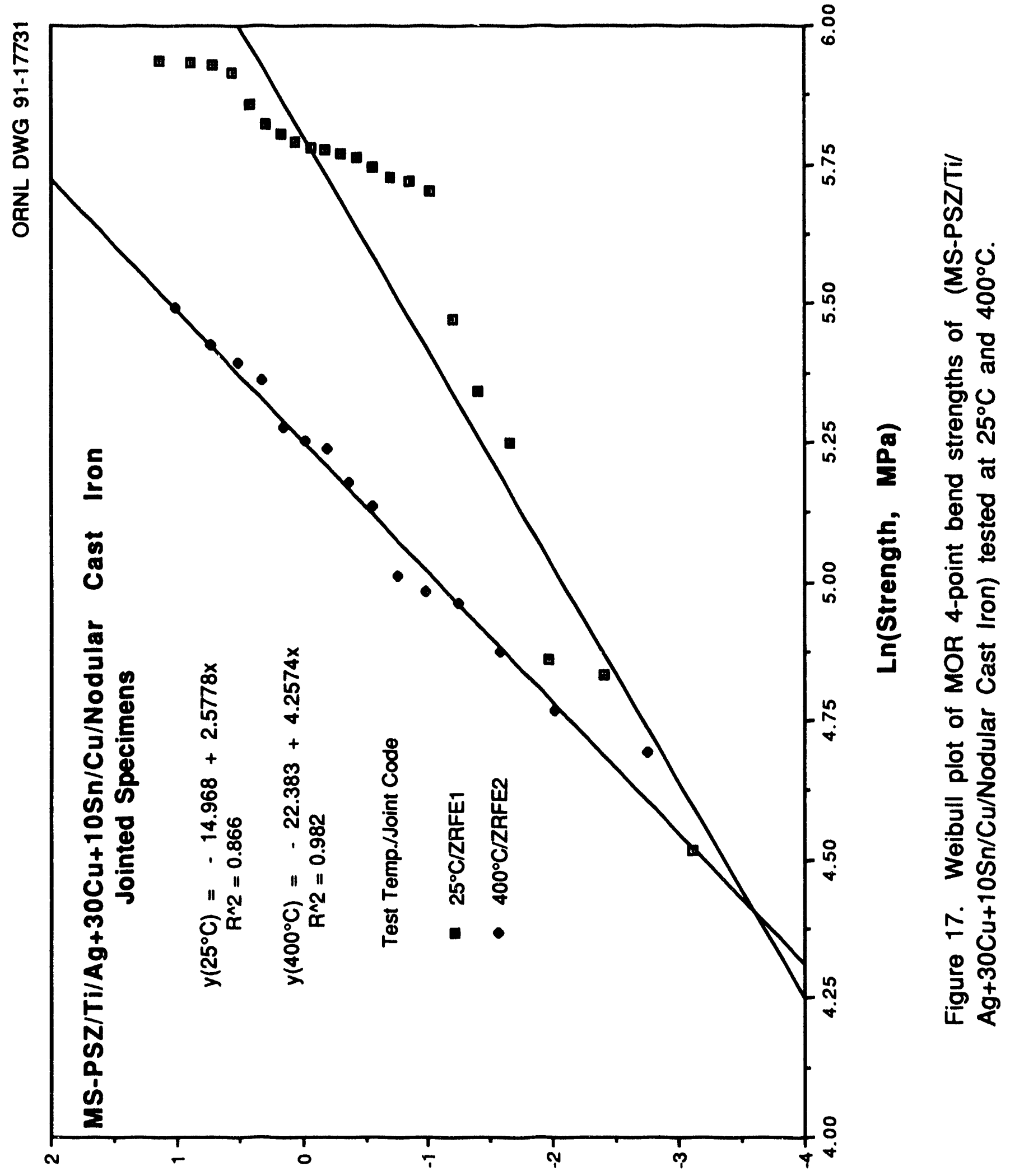

$[(y-L) / L] \cup 747$ 


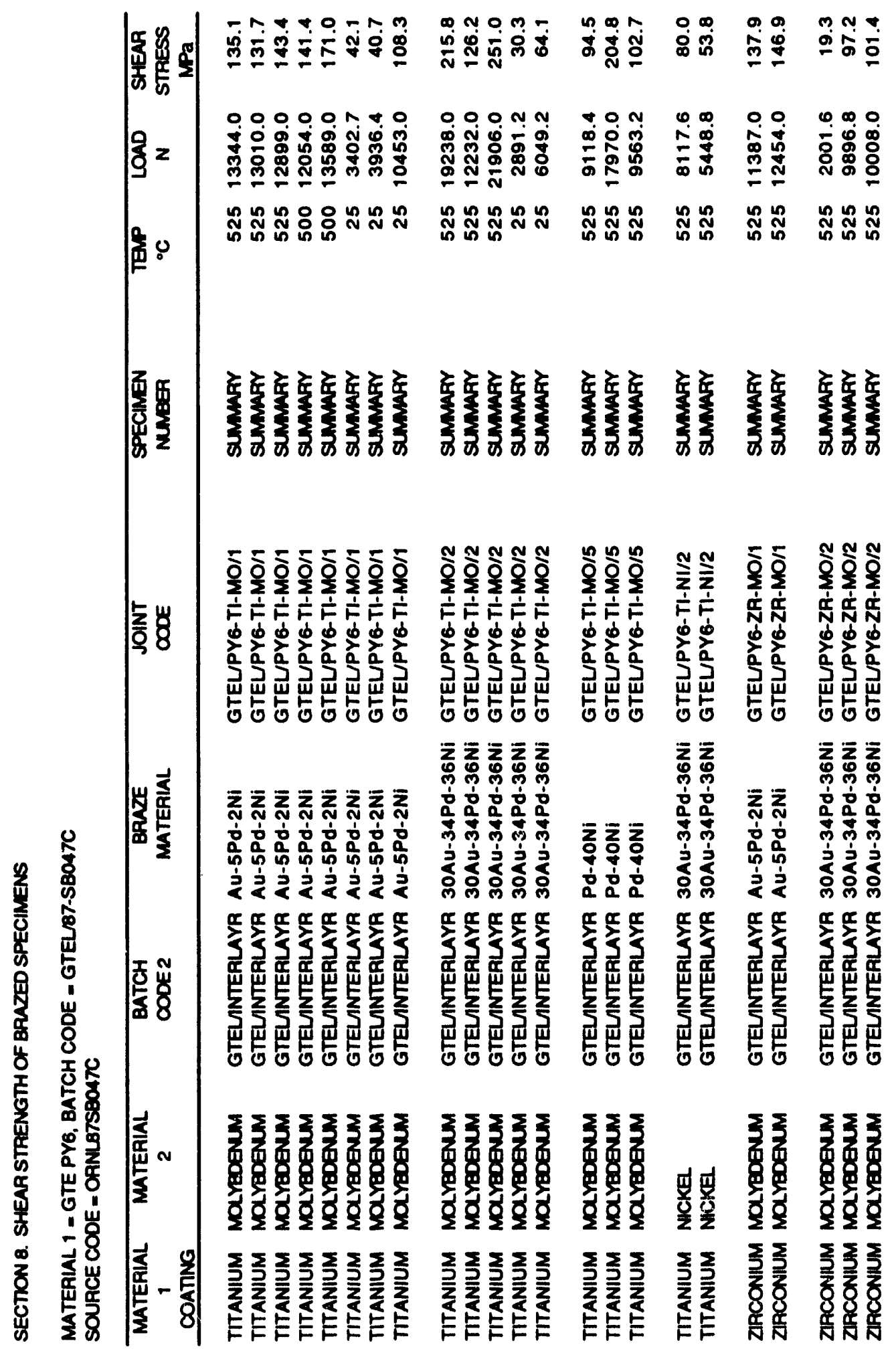




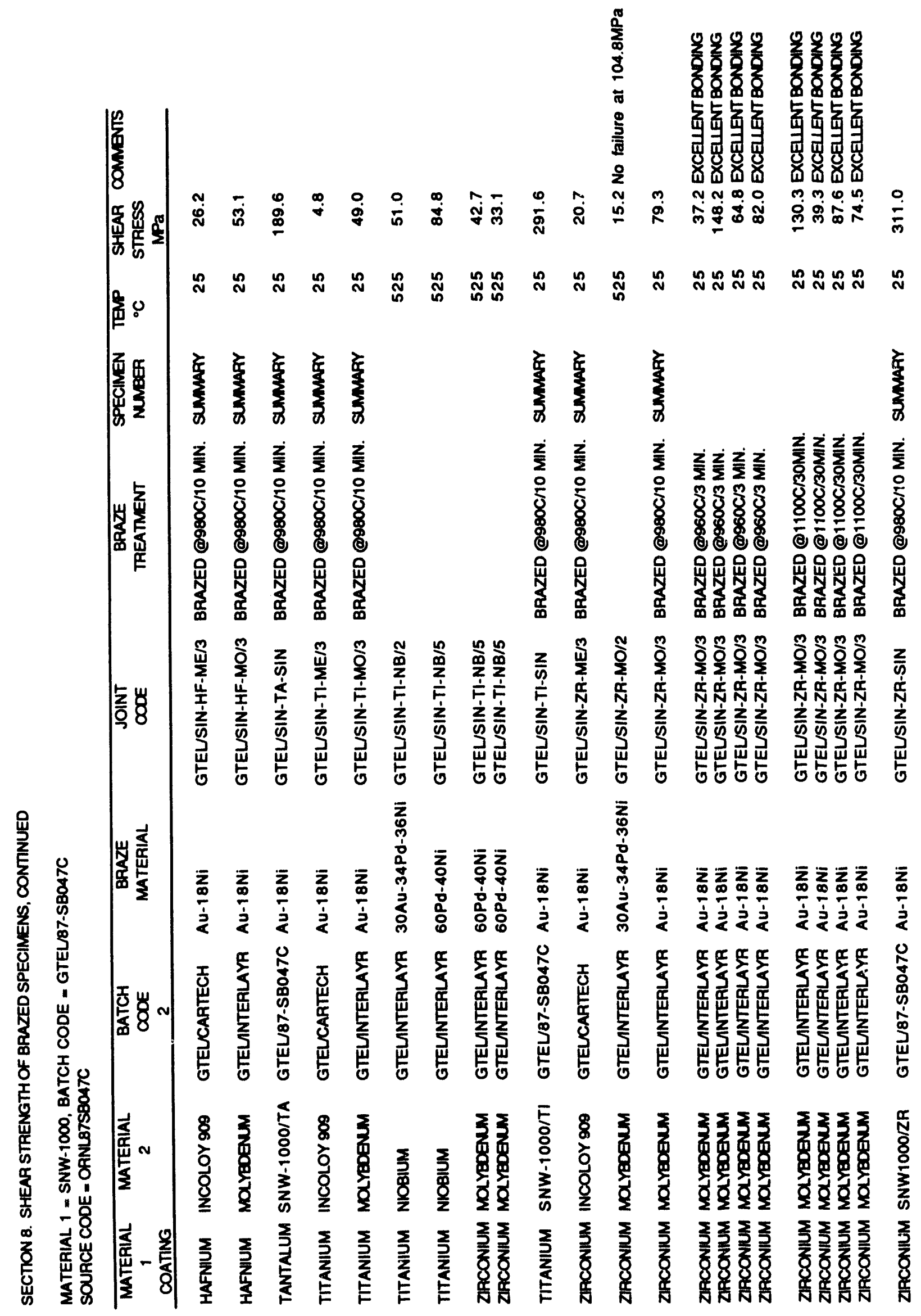




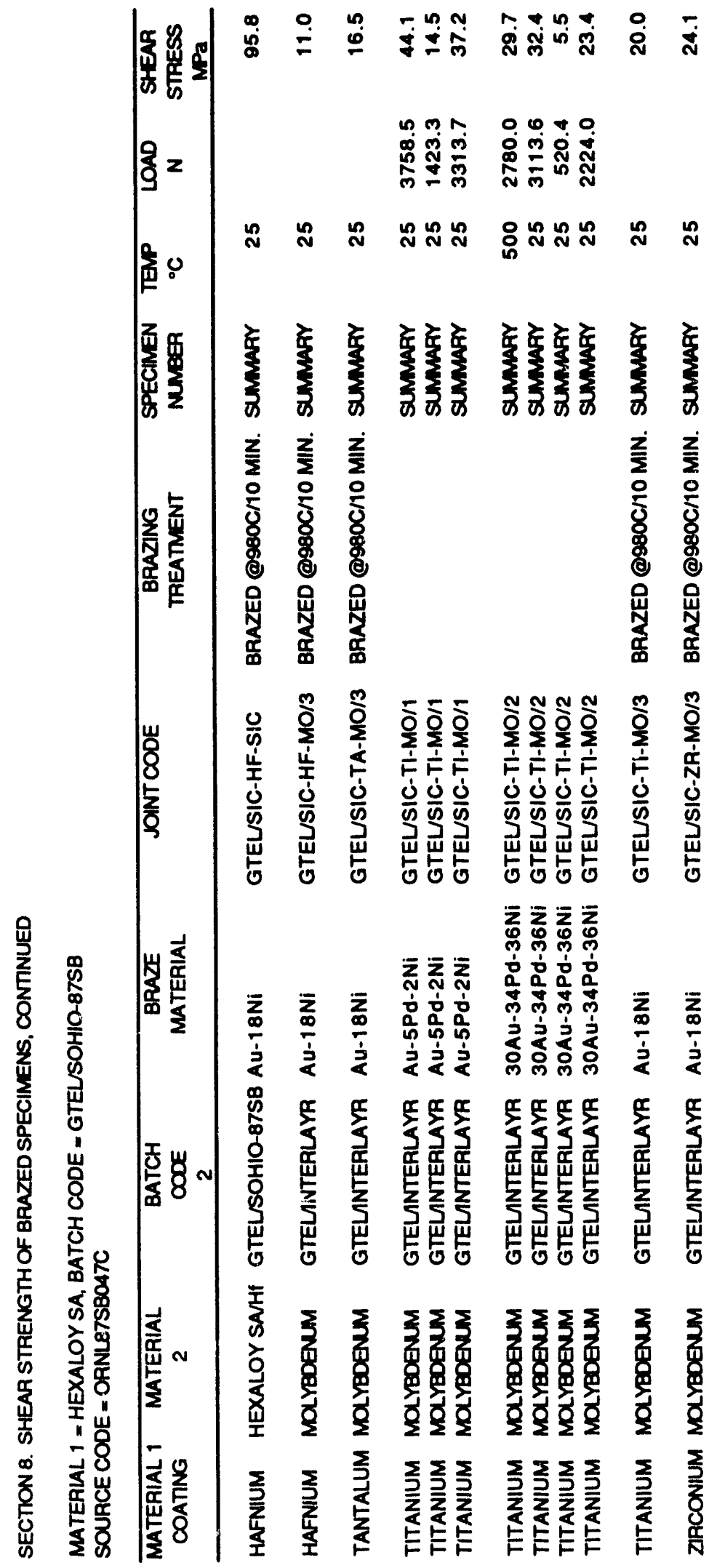




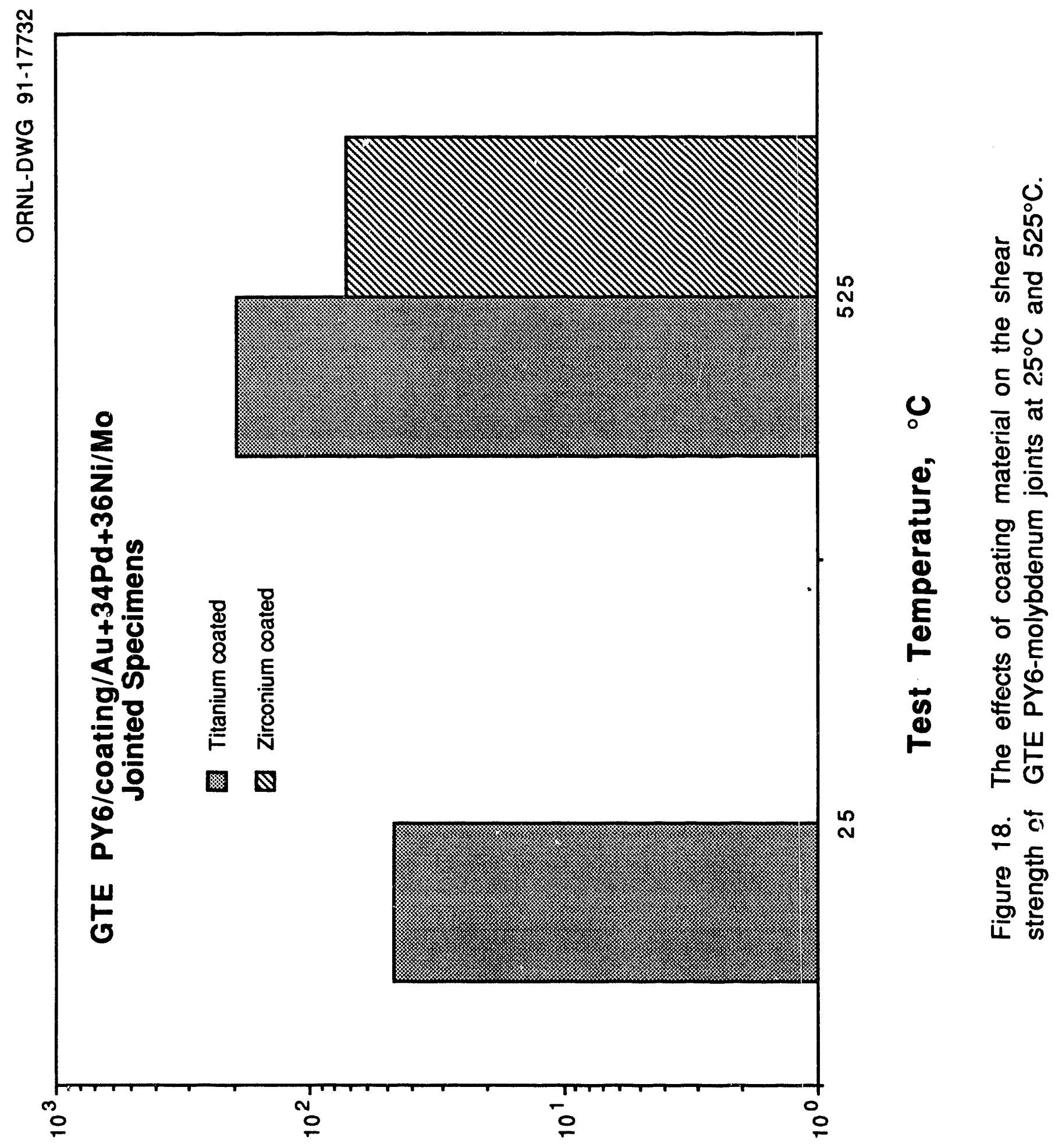

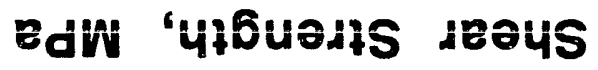




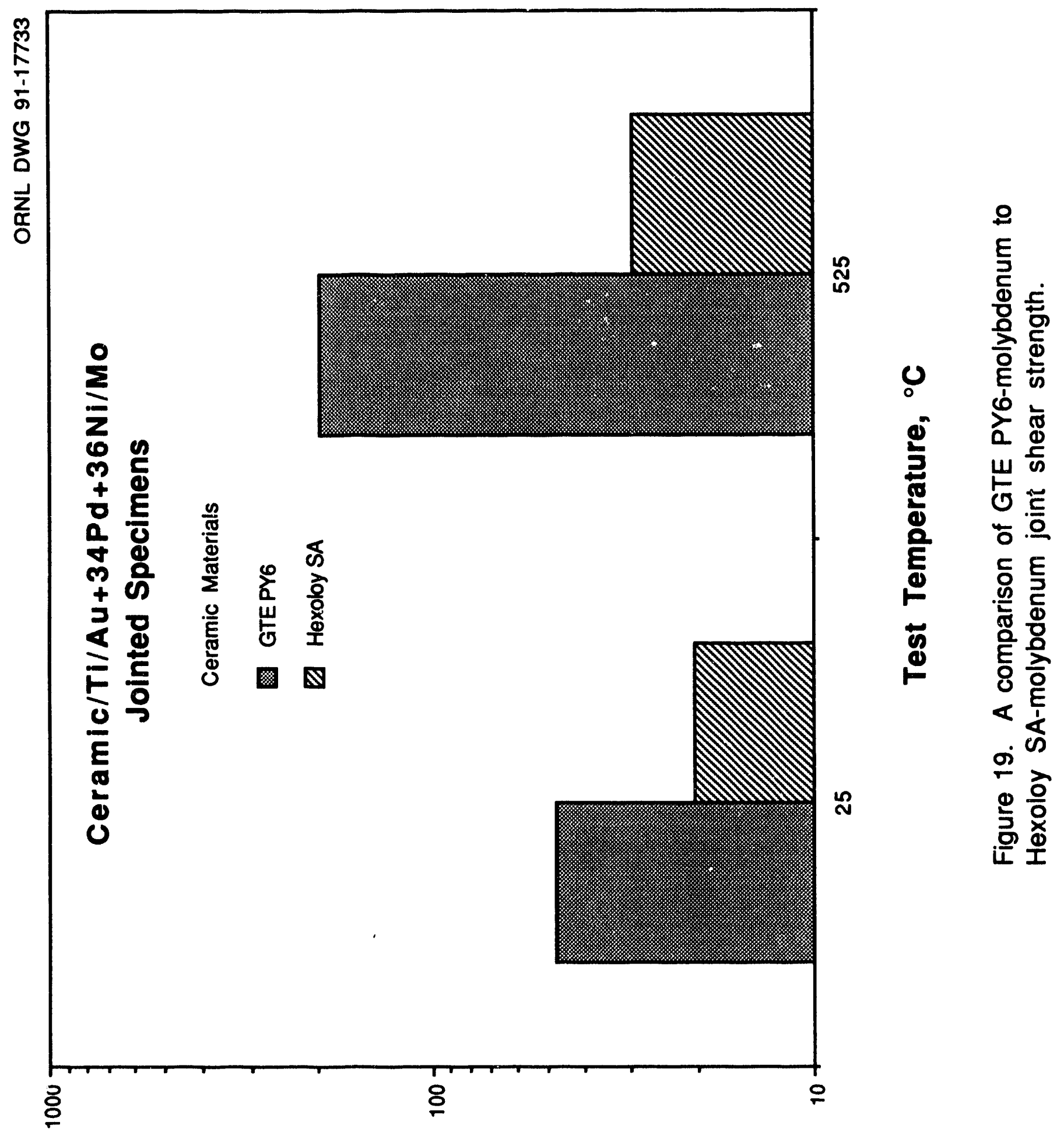

edW "पłôuədt deəuS 


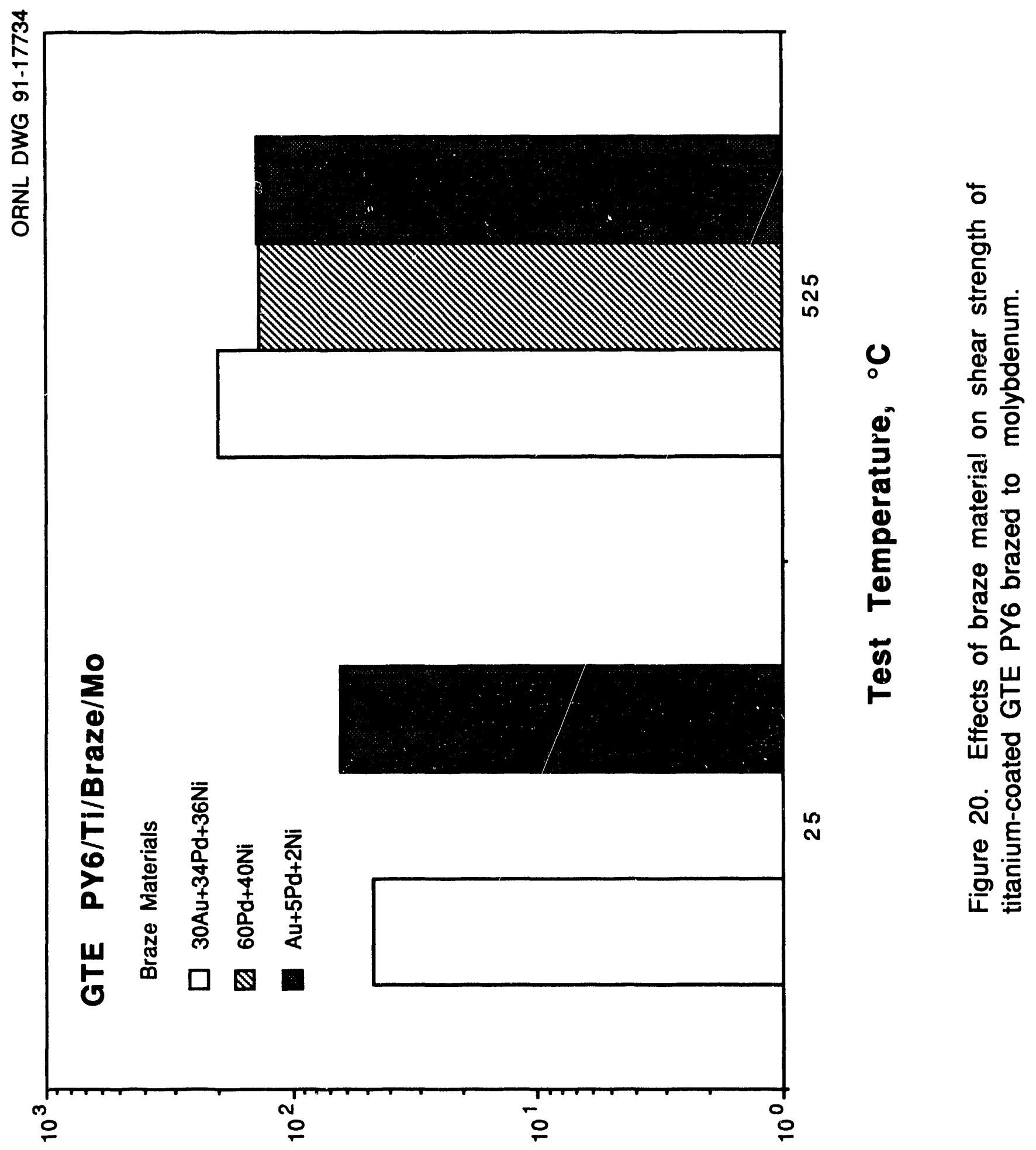

EdW '4ibuajis deaus 
SECTION 9. TORSION OF BRAZED SPECIMENS

MATERIAL $1=$ HEXALOY SA coated with titanium, BATCH CODE = GTELSOHIO-87SB

MATERIAL 2 = INCOLOY 909, BATCH CODE = GTEL $C A R T E C H$

BRAZE MATERIAL = AU-5Pd-2NI

SOURCE CODE = ORNL87SB047C

JOINT CODE = GTELSIC-INCSO9

\begin{tabular}{ccccl}
\hline $\begin{array}{c}\text { TEST } \\
\text { TEMP } \\
{ }^{\circ} \mathrm{C}\end{array}$ & $\begin{array}{c}\text { TORQUE } \\
\mathrm{Nm}\end{array}$ & $\begin{array}{c}\text { BENDING } \\
\text { MOMENT } \\
\mathrm{Nm}\end{array}$ & $\begin{array}{c}\text { ROTATION } \\
\text { DEGREES }\end{array}$ & \multicolumn{1}{c}{ COMMENTS } \\
\hline 25 & 11.980 & 18.350 & 0.25 & BROKE IN CERAMIC \\
25 & 7.910 & 6.100 & 0.25 & BROKE IN CERAMIC \\
25 & 7.680 & 6.100 & 0.30 & BROKE IN CERAMIC \\
650 & 1.130 & & & SLIPPED AT BRZCER INTFC \\
650 & 2.820 & & & SLIPPED AT BRZICER INTFC \\
650 & 4.520 & & & SLIPPED AT BRZCER INTFC \\
\hline
\end{tabular}

MATERIAL 1 = GTE PY6 coaled with titanium, BATCH CODE = GTEL/87-SB047C

MATERIAL 2 = INCOLOY 909, BATCH CODE = GTEL/CARTECH

BRAZE MATERIAL $=$ AU-5Pd-2NI

SOURCE CODE = ORNL87SBO47C

JOINT CODE = GTELPYG-INC909

\begin{tabular}{ccccl}
\hline $\begin{array}{c}\text { TEST } \\
\text { TEMP } \\
{ }^{\circ} \mathrm{C}\end{array}$ & $\begin{array}{c}\text { TORQUE } \\
\mathrm{Nm}\end{array}$ & $\begin{array}{c}\text { BENDING } \\
\text { MOMENT } \\
\mathrm{Nm}\end{array}$ & $\begin{array}{c}\text { ROTATION } \\
\text { DEGREES }\end{array}$ & COMMENTS \\
\hline 25 & 60.900 & 7.120 & 2.22 & BROKE IN CERAMIC \\
25 & 97.390 & 14.240 & 3.52 & BROKE IN CERAMIC \\
25 & 53.100 & 11.190 & 1.98 & BROKE IN CERAMIC \\
25 & 30.170 & 13.220 & 1.15 & BROKE IN CERAMIC \\
25 & 59.660 & 10.170 & 1.05 & BROKE IN CERAMIC \\
650 & 4.630 & & & SLIPPED AT BRZCER INTFC \\
650 & 1.580 & & 0.10 & SLIPPED AT BRZICER INTFC \\
650 & 7.010 & & & SLIPPED AT BRZCER INTFC \\
650 & 4.860 & & 0.21 & SLIPPED AT BRZCER INTFC \\
\hline
\end{tabular}

MATERIAL 1 = GTE PY6 coated with thanium, BATCH CODE = GTEL87-SB047C

MATERIAL 2 = INCONEL 718, BATCH CODE = GTELHUNTALLOY

BRAZE MATERIAL = AU-5Pd-2Ni

SOURCE CODE = ORNL87SB047C

JOINT CODE = GTEUPYG-INC718

\begin{tabular}{ccccl}
\hline $\begin{array}{c}\text { TEST } \\
\text { TEMP } \\
{ }^{\circ} \mathrm{C}\end{array}$ & $\begin{array}{c}\text { TORQUE } \\
\mathrm{Nm}\end{array}$ & $\begin{array}{c}\text { BENDING } \\
\text { MOMENT } \\
\mathrm{Nm}\end{array}$ & $\begin{array}{c}\text { ROTATION } \\
\text { DEGREES }\end{array}$ & COMMENTS \\
\hline 25 & 12.200 & 25.420 & 0.38 & BROKE IN CERAMIC \\
25 & 39.540 & 13.220 & 1.19 & BROKE IN CERAMIC \\
25 & 22.600 & 17.290 & 0.70 & BROKE IN CERAMIC \\
25 & 51.180 & 16.270 & 0.80 & BROKE IN CERAMIC \\
25 & 40.670 & 16.270 & 0.64 & BROKE IN CERAMIC \\
950 & 0.340 & & & SLIPPED AT BRZCER INTFC
\end{tabular}


SECTION 10. TORSION FATIGUE OF BRAZED SPECIMENS

MATERIAL 1 = GTE PY6 coated with titanium, BATCH CODE = GTEL/87-SB047C

MATERIAL 2 = INCOLOY 909, BATCH CODE = GTELCARTECH

BRAZE MATERIAL $=$ AU-5Pd-2Ni

SOURCE CODE $=$ ORNL87SBO47C

JOINT CODE = GTEL/PY6-INC909

\begin{tabular}{lccccl}
\hline $\begin{array}{c}\text { SPECIMEN } \\
\text { NUMBER }\end{array}$ & $\begin{array}{c}\text { TEMP } \\
{ }^{\circ} \mathrm{C}\end{array}$ & $\begin{array}{c}\text { TORQUE } \\
\mathrm{Nm}\end{array}$ & $\begin{array}{c}\text { BENDING } \\
\text { MONENT } \\
\mathrm{Nm}\end{array}$ & $\begin{array}{c}\text { ROTATION } \\
\text { DEGPEES }\end{array}$ & $\begin{array}{c}\text { CYCLESTO } \\
\text { FAILURE }\end{array}$ \\
\hline BZTRFT1* & 25 & 4 TO 20.9 & 20.300 & +-0.31 & $>1000$ \\
BZTRFT2* & 25 & 4 TO 20.9 & 19.300 & +-0.32 & $>1000$ \\
BZTRFT3* & 25 & 4 TO 20.9 & 21.354 & +-0.31 & $>1000$ \\
& & & & & \\
BZTRFT4t & 25 & 4 TO 20.9 & 26.438 & +-0.32 & $>1000$ \\
& 25 & 4 TO 20.9 & 16.270 & +-0.32 & $>1000000$
\end{tabular}

TEST NOTES

- Specimen was heated to 650-700C, gripped in the MTS machine, then cooled before test to realign the specimen.

† Specimen was heated to 650-700C, gripped in the MTS machine, then cooled before testing to realign the specimen. This test was run until fracture, enduring more than $1,000,000$ cycles with no signs of degradation. Parameters unchanged from beginning of the test. 


\section{INTERNAL DISTRIBUTION}

1-2. Central Research Library

3. Document Reference Section

4-5. Laboratory Records Department

6. Laboratory Records, ORNL RC

7. ORNL Patent Section

8-10. M\&C Records Office

11. L. F. Allard, Jr.

12. L. D. Armstrong

13. P. F. Becher

14. R. F. Bernal

15. T. M. Besmann

16. P. J. Blau

17. A. Bleier

18. E. E. Bloom

19. K. W. Boling

20. R. A. Bradley

21. C. R. Brinkman

22. V. R. Bullington

23. R. S. Carlsmith

24. P. T. Carlson

25. G. M. Caton

26. S. J. Chang

27. R. H. Cooper, Jr.

28. B. L. Cox

29. D. F. Craig

30. S. A. David

31. J. H. DeVan

32. J. L. Ding

33. M. K. Ferber

34. F. M. Foust

35. W. Fulkerson

36. R. L. Graves

37. D. L. Greene

38. M. H. Harris

39. E. E. Hoffman

40. C. R. Hubbard

41. M. A. Janney
42. M. G. Jenkins

43. D. R. Johnson

44. W. F. Jones

45. D. Joslin

46. R. R. Judkins

47. M. A. Karnitz

48. M. R. Kass

49-53. B. L. P. Keyes

54. H. D. Kimrey, Jr.

55. T. G. Kollie

56. K. C. Liu

57. E. L. Long, Jr.

58. W. D. Maniy

59. R. W. MCClung

60. D. J. McGuire

61. J. R. Merriman

62. D. L. Moses

63. T. A. Nolan

64. A. E. Pasto

65. J. L. Rich

66. C. R. Richmond

67. J $M$ Robbins

68. M. L. Santella

69. A. C. Schaffhauser

70. S. Scott

71. G. M. Slaughter

72. E. J. Soderstrom

73. D. P. Stinton

74. R. W. Swindeman

75. V. J. Tennery

76. T. N. Tiegs

77. J. R. Weir, Jr.

78. B. H. West

79. F. W. Wiffen

80. S. G. Winslow

81. J. M. Wyrick

82. C. S. Yust 


\section{EXTERNAL DISTRIBUTION}

83. James H. Adair

University of Florida

Materials Science and

Engineering

317 MAE B1dg.

Gainesville, FL 32611-2066

84. Donald F. Adams

University of Wyoming

Mechanical Engineering

Department

P. 0. Box 3295

Laramie, WY 82071

85. Jalees Ahmad

AdTech Systems Research, Inc.

1342 North Fairfield Road

Dayton, $\mathrm{OH}$ 45432-2698

86. Yoshio Akimune

NISSAN Motor Co., Ltd.

Materials Research Laboratory

1 Natsushima-Cho

Yokosuka 237

JAPAN AIR MAIL

87. Mufit Akinc

Iowa State University

322 Spedding $\mathrm{Hall}$

Ames, IA 50011

88. Ilhan A. Aksay

University of Washington

Materials Science and Engineering Department, FB-10

Seattle, WA 98195

89. R. G. Alexander

BASE

$26 \mathrm{Malvern}$ Close

Kettering Northants NN16 AJP

UNITED KINGDOM AIR MAI:-

90. Richard L. Allor

Ford Motor Company

Material Systems

Reliability Department

20000 Rotunda Drive

P.0. Box 2053, Room S-2031

Dearborn, MI 48121-2053
91. Richard T. Alpaugh

U.S. Department of Energy

Advanced Propulsion Division

CE-322, Forrestal Building

Washington, DC 20585

92. Joseph E. Amaral

Instron Corporation

Corporate Engineering Office

100 Royale Street

Canton, MA 02021

93. Edward M. Anderson

Aluminum Company of America

North American Industrial Chemicals Division

P.0. Box 300

Bauxite, AR 72011

94. Norman C. Anderson

Ceradyne, Inc.

Ceramic-to-Metal Division

3169 Redhill Avenue

Costa Mesa, CA 92626

95. Don Anson

Battelle Columbus Laboratories

Thermal Power Systems

505 King Avenue

Columbus, $\mathrm{OH}$ 43201-2693

96. Thomas Arbanas

G.B.C. Materials Corporation

580 Monastery Drive

Latrobe, PA 15650-2698

97. Frank Armatis

3M Company

$3 M$ Center

Building 60-1N-01

St. PauT, MN 55144-1000

98. Everett B. Arnold

Detroit Diesel Corporation

Mechanical Systems Technology

13400 Outer Drive, West

Detroit, MI 48239-4001

99. Richard M. Arons

PA Consulting Group

279 Princeton Road

Hightstown, NJ 08550 
100. Berti1 Aronsson

Sandvik $A B$

S-12680

Stockholm Lerkrogsvagen 19

SWEDEN AIR MAIL

101. Dennis Assanis

University of Illinois

Department of Mechanical

Engineering

1206 W. Green Street

Urbana, IL 61801

102. William H. Atwell

Dow Corning Corporation

3901 South Saginaw Road

MS: 540

Midl and, MI 48686-0995

103. V. S. Avva

North Carolina A\&T State University

Department of Mechanical Engineering

Greensboro, NC 27411

104. Patrick Badgley

Adiabatics, Inc.

3385 Commerce Drive

Columbus, IN 47201

105. Sunggi Baik

Pohang Institute of Science \& Technology

Department of Materials Science and Engineering

P.0. Box 125

Pohang 790-600

KOREA AIR MAIL

106. John M. Bailey

Caterpiliar, Inc.

Technical Center

Building $L$

P.0. Box 1875

Peoria, IL 61656-1875

107. Bob Baker

Ceradyne, Inc.

3169 Redhill Avenue

Costa Mesa, CA 92626
108. Frank Baker

Aluminum Company of America

Alcoa Technical Center

Alcoa Center, PA 15069

109. J. G. Baldoni

GTE Laboratories Inc.

40 Sylvan Road

Waltham, MA 02254

110. Clifford P. Ballard

Allied-Signal, Inc.

Ceramics Program

P.0. Box 1021

Morristown, NJ 07962-1021

111. M. Balu

Amoco Performance Products Inc. 4500 McGinnis Ferry Road

Alpharetta, GA 30202

112. B. P. Bandyopadhyay

Toyohashi University of Technology

School of Production Systems Engineering

Tempaku-Cho Toyohashi 440

JAPAN AIR MAIL

113. P. M. Barnard

Ruston Gas Turbines Limited

Metallurgical Laboratory

P. 0. Box 1

Lincoln LN2 5DJ

ENGLAND AIR MAIL

114. Harold N. Barr

Hittman Corporation

9190 Red Branch Road

Columbia, MD 21045

115. Renald D. Bartoe

Vesuvius McDanel

510 Ninth Avenue

Box 560

Beaver Falls, PA 15010-0560

116. Donald M. Bartos

Dow Corning Corporation

Advanced Ceramics Program

Midland, MI 48686-0995 
117. David L. Baty

Babcock \& Wilcox-LRC

P.0. Box 11165

Lynchburg, VA 24506-1165

118. Donald F. Baxter, Jr.

ASM International

Advanced Materials and

Processes

Materials Park, OH 44073-0002

119. M. Brad Beardsley

Caterpillar, Inc.

Advanced Materials Technology

Technical Center Bldg. E

P.0. Box 1875

Peoria, IL 61656-1875

120. Robert Beck

Teledyne CAE

Materials Engineering/Programs

1330 Laskey Road

P.0. Box 6971

Toledo, OH 43612

121. John C. Bell

Shell Research Limited

Thornton Resea.ch Centre

P.0. Box 1

Chester, Chl 3SH

ENGLAND AIR MAIL

122. Albert H. Bell, III

General Motors Technical Center 30200 Mound Road

Engineering Building/W3 Turbine

Warren, MI 48090-9010

123. M. Bentele

Xamag, Inc.

259 Melville Avenue

Fairfield, CT 06430

124. Larry D. Bentsen

BF Goodrich Company

R\&D Center

9921 Brecksville Road

Brecksville, OH 44141

125. Joseph C. Bentz

ENCERATEC

2525 Sandcrest Drive

Columbus, IN 47203
126. Louis Beregszazi

Defiance Precision Products

P.0. Drawer 428

Defiance, $\mathrm{OH} 43512$

127. Tom Bernecki

Northwestern University

BIRL

1801 Maple Avenue

Evanston, IL 60201-3135

128. Charles F. Bersch

Institute for Defense Analyses

1801 North Beauregard Street

Alexandria, VA 22311

129. Ram Bhatt

NASA Lewis Research Center

21000 Brookpark Road

Clevel and, $\mathrm{OH} 44135$

130. Deane I. Biehler

Caterpillar, Inc.

Engineering and Research

Materials

Technical Center, Building $E$

P.0. Box 1875

Peoria, IL 61656-1875

131. John. W. BjerkTie

Consolidated Natural Gas

Service Co., Inc.

Research Department

CNG Tower

Pittsburgh, PA 15222-3199

132. William D. Bjorndah1

$T R W$, Inc.

One Space Park

Building 01, Room 2040

Redondo Beach, CA 90278

133. Keith A. Blakely

Advanced Refractory

Technologies, Inc.

699 Hertel Avenue

Buffalo, NY 14207

134. Edward G. Blanchard

Netzsch Inc.

119 Pickering Way

Exton, PA 19341 
135. Keith $B$ landford

Boride Products, Inc.

2879 Aero Park Drive

Traverse City, MI 49684

136. Bruce Boardman

Deere and Company Technical

Center

3300 River Drive

Moline, IL 61265

137. Russell Bockstedt

Hoechst Celanese Corporation

150 JFK Parkway

Short Hills, NJ 07078

138. M. Boehmer

DLR German Aerospace Research Establ ishment

Postfach 906058

D-5000 Koln 90

GERMANY AIR MAIL

139. Lawrence P. Boesch

EER Systems Corp.

1593 Spring Hill Road

Vienna, VA 22182-2239

140. Donald H. Boone

Boone \& Associates

2412 Cascade Drive

Walnut Creek, CA 94598-4313

141. Tom Booth

Allied-Signal Aerospace

Company

AiResearch Los Angeles

Division

2525 West 190th Street

Torrance, CA 90509-2960

142. Tibor Bornemisza

Sundstrand Power Systems

4400 Ruffin Road

San Diego, CA 92186-5757

143. J.A.M. Boulet

University of Tennessee

Department of Engineering

Science and Mechanics

310 Perkins Hall

Knoxville, TN 37996-2030
144. H. Kent Bowen

Massachusetts Institute of

Technology

77 Massachusetts Avenue

Room E40-434

Cambridge, MA 02139

145. Leslie J. Bowen

Materials Systems

53 Hillcrest Road

Concord, MA 01742

146. Steven C. Boyce

Air Force Office of

Scientific Research

AFOSR/NA Bldg. 410

Bolling AFB DC 20332-6448

147. Gary L. Boyd

CEC

400 Howell Avenue, No. 4

Warland, WY 82401

148. Steve Bradley

UOP Research Center

50 East Algonquin Road

Des Plaines, IL 60017-6187

149. Michael C. Brands

Cummins Engine Company, Inc.

P.0. Box 3005

Mall Code 50179

Columbus, IN 47201

150. Raymond J. Bratton

Westinghouse Science and

Technology Center

1310 Beulah Road

Pittsburgh, PA 15235

151. John J. Brennan

United Technologies Corporation

Research Center

Silver Lane, MS: 24

East Hartford, CT 06108

152. Jeff D. Bright

Ceramatec, Inc.

2425 South 900 West

Salt Lake City, UT 84108 
153. Terrence K. Brog Coors Ceramics Company

Corporate Development and Technology

4545 McIntyre Street

Golden, CO 80403

154. Gunnar Broman

317 Fairlane Drive

Spartanburg, SC 29302

155. Al Brown

High-Tech Materials Alert

P.0. Box 882

Dayton, NJ 08810

156. Jesse Brown

Virginia Polytechnic Institute and State University

Center for Advanced Ceramic Materials

Blacksburg, VA 24061-0256

157. Sherman D. Brown

University of Illinois

Materials Science and

Engineering Department

105 South Goodwin Avenue

204 Ceramics Building

Urbana, IL 61801

158. S. L. Bruner

Ceramatec, Inc.

2425 South 900 West

Salt Lake City, UT 84119

159. Adolfo Brusaferro

Keramont Corporation

4231 South Fremont Avenue

Tucson, AZ 85714

160. W. Bryzik

U.S. Army Tank Automotive Command

R\&D Center

Propulsion Systems Division Warren, MI 48397-5000

161. S. T. Buljan

GTE Laboratories, Inc.

40 Sylvan Road

Waltham, MA 02254
162. S. J. Burden

GTE Valenite

1711 Thunderbird

Troy, MI 48084

163. Curt V. Burkland

AMERCOM, InC.

8928 Fullbright Avenue

Chatsworth, CA 91311

164. Bill Bustamante AMERCOM, Inc.

8928 Fullbright Street

Chatsworth, CA 91311

165. Oral Buyukozturk

Massachusetts Institute of Technology

77 Massachusetts Avenue

Room 1-280

Cambridge, MA 02139

166. David A. Caillet

Ethyl Corporation

451 Florida Street

Baton Rouge, LA 70801

167. Frederick J. Calnan

Heany Industries, Inc.

249 Briarwood Lane

P.0. Box 38

Scottsville, W 14546

168. Roger Cannon

Rutgers University

Ceramics Department

P. 0. Box 909

Piscataway, NJ 08855-0909

169. Scott Cannon

P.0. Box 567254

Atlanta, GA 30356

170. Harry W. Carpenter

19945 Acre Street

Northridge, CA 91324

171. David Carruthers

Kyocera Industrial Ceramics Company

P.0. Box 2279

Vancouver, WA 98668-2279 
172. Calvin H. Carter, Jr. Cree Research, Inc. 2810 Meridian Parkway Durham, NC 27713

173. J. David Casey 35 Atlantis street West Roxbury, MA 02132

174. Jere G. Castor J. C. Enterprise 5078 North 83rd Street Scottsdale, AZ 85250

175. James D. Cawley Case Western Reserve University Materials Science and Engineering Department Cleveland, $\mathrm{OH} 44106$

176. Thomas C. Chadwick Den-Mat Corporation P.0. Box 1729

Santa Maria, CA 93456

177. Ronald H. Chand Chand Kare Technical Ceramics 2 Coppage Drive Worcester, MA 01603

178. Robert E. Chaney EG\&G Idaho, Inc.

Idaho National Engineering Laboratory

P.0. Box 1625

Idaho Falls, ID 83415-3525

179. Frank C. Chang

U.S. Army Materials Technology Laboratory

AMTL-EMM

405 Arsenal Street

Watertown, MA 02172

180. Robert M. Chapman

Allied-Signal Aerospace Company

1530 Wilson Boulevard, 10th Floor

Arlington, VA 22209
181. William Chapman

Williams International Corporation 2280 West Maple Road Walled Lake, MI 48390-0200

182. Charlife Chen LECO Corporation P.0. Box 211688

Augusta, GA 30917

183. Albert A. Chesnes

U.S. Department of Energy Transportation Technologies CE-30, Forrestal Building, 6B-094 Washington, DC 20585

184. Kaiyin Chia

Carborundum Company

P.0. Box 832

Niagara Falls, NY 14302

185. Frank Childs

EG\&G Idaho, Inc.

Idaho National Engineering Laboratory

P.0. Box 1625

Idaho Falls, ID 83415-3527

186. William J. Chmura

Torrington Company

59 Field Street

Torrington, CT 06790-4942

187. Tsu-Wei Chou

University of Delaware

Center for Composite Materials

201 Spencer Laboratory

Newark, DE 19716

188. R. J. Christopher

Ricardo Consulting Engineers

Bridge Works

Shoreham-By-Sea West Sussex BN43 5FG

ENGLAND AIR MAIL

189. Joel P. Clark

Massachusetts Institute of Technology

Room 8-409

Cambridge, MA 02139 
190. Giorgio Clarotti

Commission of the European Communities

DGXII-C3, M075, 1-53;

200 Rue de la Loi

B-1049 Brussels

BELGIUM AIR MAIL

191. W. J. Clegg

ICI Advanced Materials

P.0. Box 11

The Heath, Runcorn Cheshire

WA7 4QE

ENGLAND

AIR MAIL

192. Joseph Cleveland

GTE Products Corporation

Hawes Street

Towanda, PA 18848-0504

193. Gloria M. Collins

ASTM

1916 Race Street

Philadelphia, PA 19103

194. William C. Connors

Sundstrand Aviation Operations

Materials Science and

Engineering Department

4747 Harrison Avenue

P.0. Box 7002

Rockford, IL 61125-7002

195. John A. Coppola

Carborundum Company

P.0. Box 156

Niagara Falls, NY 14302

196. Normand D. Corbin

Norton Company

Advanced Ceramics

Goddard Road

Northboro, MA 01532-1545

197. Douglas Corey

Allied-Signal Aerospace

Company

2525 West 190th Street

MS: T52

Torrance, CA 90504-6099
198. Keith P. Costello

Chand/Kare Technical Ceramics

2 Coppage Drive

Worcester, MA 01603-1252

199. Ed L. Courtright

Pacific Northwest Laboratory

MS:K3-59

Richland, WA 99352

200. Anna Cox

Mitchell Market Reports

P.0. Box 23

Monmouth Gwent NP5 4YG

UNITED KINGDOM AIR MAIL

201. Thomas Coyle

Unocal Corporation

Unocal Science \& Technology Division

376 South Valencia Avenue

Brea, CA 92621

202. Art Cozens

Instron Corporation

3414 Snowden Avenue

Long Beach, CA 90808

203. Robert C. Craft

American Ceramic Society, Inc.

757 Brooksedge Plaza Drive

Westerville, $\mathrm{OH} 43081$

204. Mark Crawford

New Technology Week

4604 Monterey Drive

Annandale, VA 22003

205. Richard A. Cree

Markets \& Products, Inc.

P.0. Box 14328

Columbus, $\mathrm{OH}$ 43214-0328

206. Les Crittenden

Vesuvius McDanel

Box 560

Beaver Falls, PA 15010 
207. William J. Croft

U.S. Army Materials Technology Laboratory 405 Arsenal Street Watertown, MA 02172

208. M. J. Cronin Mechanical Technology, Inc. 968 Albany-Shaker Road Latham, NY 12110

209. Gary M. Crosbie

Ford Motor Company 1430 Culver Avenue S-2079, SRL Building Dearborn, MI 48121-4036

210. Floyd W. Crouse, Jr. U.S. Department of Energy Morgantown Energy Technology Center Collins Ferry Road P.0. Box 880 Morgantown, WV 26505

211. John Cuccio

Allied-Signal Aerospace Company

Garrett Auxiliary Power Division

P.0. Box 5227, MS: 1302-2Q

Phoenix, AZ 85010

212. Raymond Cutler

Ceramatec, Inc.

2425 South 900 West

Salt Lake City, UT 84119

213. Charles D'Angelo

GTE Labcratories, Inc.

40 Sylvan Road

Wal tham, MA 02154

214. Stephen C. Danforth

Rutgers University

Ceramic Engineering Department P.0. Box 909

Piscataway, NJ 08855-0909
215. Sankar Das Gupta

Electrofuel Manufacturing Co., Ltd.

9 Hanna Avenue

Toronto Ontario MGK-1W8

CANADA AIR MAIL

216. Charles Davis

Sverdrup Technology, Inc., MSFC

620 Discovery Drive

Huntsville, AL 35806

217. Frank Davis

Allied Signal Aerospace Co.

7550 Lucerne Dr., \# 203

Middleburg Heights, $\mathrm{OH} 44130$

218. Robert F. Davis

North Carolina State University

Materials Engineering Department

229 Riddick Laboratory

P.0. Box 7907

Raleigh, NC 27695

219. Thomas DeAngel is

Carborundum Company

Niagara Falls R\&D Center

P.0. Box 832

Niagara Falls, NY 14302

220. George DeBell

Ford Motor Company

Material Systems Reliability Department

20000 Rotunda Drive

P.0. Box 2053, Room S-2023

Dearborn, MI 48121-2053

221. Michael DeLuca

AMP-AKZO

West Lane

Aquebogue, NY 11931

222. Gerald L. DePoorter

Colorado School of Mines

Metallurgical and Materials

Engineering Department

Golden, CO 80401 
223. J. $\bar{r}$. DeRidder

Omni Electro Motive, Inc., 12 Seely Hill Road

Newfield, NY 14867

224. Nick C. Dellow

Materials Technology

Publications

40 Sotheron Road

Watford Herts WD1 2QA

UNITED KINGDOM AIR MAIL

225. L. R. Dharani

University of Missouri-Rolla

224 M.E.

Rolla, MO 65401

226. Douglas A. Dickerson

Union Carbide Specialty

Powders

1555 Main Street

Indianapolis, IN 46224

227. John Dodsworth

Vesuvius Research \&

Development

Technical Ceramics Group

Box 560

Beaver Falls, PA 15010

228. B. Dogan

Institut fur Werkstofforschung

GKSS-Forschungszentrum

Geesthacht GmbH

Max-Planck-Strasse

D-2054 Geesthacht

GERMANY AIR MAIL

229. Jean-Marie Drapier

FN Moteurs S.A.

Material and Processing

B-404l Milmort (Herstal)

BELGIUM AIR MAIL

230. Kenneth C. Dreitlein

United Technologies

Research Center

Silver Lane

East Hartford, CT 06108
231. Robin A.L. Drew

McGill University

Department of Mining and

Metallurgical Engineering

3450 University Street

Montreal Quebec H3A 2A7

CANADA AIR MAIL

232. Winston H. Duckworth

$\mathrm{BCL}$

Columbus Division

505 King Avenue

Columbus, OH 43201-2693

233. Edmund M. Dunn

Texel Company

6 Third Street

Lexington, MA 02173

234. Bill Durako

Sundstrand Aviation Operations

Department 789-6

4747 Harrison Avenue

P.0. Box 7002

Rockford, IL 61125-7002

235. Ernest J. Duwell

212 Elm Street

Hudson, WI 54016

236. Chuck J. Dziedzic

Coors Ceramics Company

Structural Products Group

17750 West 32nd Avenue

Golden, CO 80401

237. Rotert J. Eagari

Sandia National Laboratories

Engineered Materials \& Processes Certer

Org 1700

P.0. Box 5800

Albuquerque; NM 87185-5800

238. Jeffrey Eagleson

Lanxide Corporation

1001 Connecticut Avenue, N.W.

Washingcon, DC 20036 
239. Harry E. Eaton United Technologies Corporation Research Center Silver Lane East Hartford, CT 06108

240. Harvill C. Eaton

Louisiana State University

Office of Research and Economic Development 240 Thomas Boyd Hall Baton Rouge, LA 70803

2\%1. Christopher A. Ebel Carborundum Company Technology Division P.0. Box 337

Niagara Falls, NY 14302

242. J. J. Eberhardt U.S. Department of Energy Office of Transportation Materials CE-34, Forrestal Building Washington, DC 20585

243. Jim Edler

Eaton Corporation 26201 Northwestern Highway

P.0. Box 766

Southfield, MI 48037

244. William A. Ellingson Argonne National Laboratory Materials Science \& Technology Division

9700 South Cass Avenue

Argonne, IL 60439

245. Willian S. Ellis Machined Ceramics 629 N. Graham St. N. Industrial Park Bowling Green, KY 42101

246. Glen B. Engle Nuclear \& Aerospace Materials Corporation 16716 Martincoit Road Poway, CA 92064
247. Jeff Epstein

Ceramic Technologies, Inc.

2107 Jamara Lane

Houston, TX 77077

248. Kenneth A. Epstein

Dow Chemical U.S.A.

Ceramics and Advanced

Materials

800 Building

Midl and, MI 48667

249. Art Erdemir

Argonne National Laboratory

Materials and Components

Technology Division

9700 South Cass Avenue

Argonne, IL 60439

250. E. M. Erwin

Lubrizol Corporation

1819 East 225th Street

Euclid, OH 44117

251. Kenji Esaki

Toyota Technical Center U.S.A., Inc. 2000 Town Center, Suite 500 Southfield, MI 48075

252. Jose L. Escalante Ang10-Mex-Tech. Inc. 3923 N.W. 24th Street

Miami, FL 33142

253. John N. Eustis

U.S. Department of Energy Industrial Energy Efficiency Division

CE-221, Forrestal Building

Washington, DC 20585

254. Robert C. Evans

NASA Lewis Research Center Terrestrial Propulsion Office 21000 Brookpark Road, MS:86-6 Cleveland, $\mathrm{OH} 44135$

255. W. L. Everitt Kyocera International, Inc. 8611 Balboa Avenue San Diego, CA 92123 
256. Gordon Q. Evison 332 South Michigan Avenue Suite 1730

Chicago, IL 60604

257. John W. Fairbanks

U.S. Department of Energy Advanced Propulsion Division CE-322, Forrestal Building Washington, DC 20585

258. Tim Fawcett

Dow Chemical Company

Central Research, Advanced

Ceramics Laboratory

1776 Building

Midl and, MI 48674

259. Robert W. Fawley

Sundstrand Power Systems

Division of Sundstrand Corporation

4400 Ruffin Road

P.0. Box 85757

San Diego, CA 92186-5757

260. John J. Fedorchak

GTE Products Corporation

Hawes Street

Towanda, PA 18848-0504

261. Jeff T. Fenton

Vista Chemical Company

900 Threadneedle

Houston, TX 77079

262. Larry Ferrell

Babcock \& Wilcox

0ld Forest Road

Lynchburg, VA 24505

263. Raymond R. Fessier

BIRL

Industrial Research Lab

1801 Maple Avenue

Evanston, IL 60201

264. Michelle Finch

E. I. DuPont de Nemours \& Company, Inc.

Experimental Station

Information Center E302/301

Wilmington, DE 19898
265. Arthur D. Fine

United Technologies Corporation

Pratt \& Whi.tney Aircraft

400 Main Street

MS: $163-10$

East Hartford, CT 06108

266. Ross F. Firestone

Ross Firestone Company

188 Mary Street

Winnetka, IL 60093-1520

267. Sharon L. Fletcher

Arthur D. Little, Inc.

15 Acorn Park

Cambridge, MA 02140-2390

268. Thomas F. Foltz

Textron Specialty Materials

2 Industrial Avenue

Lowe11, MA 01851

269. Renee G. Ford

Materials and Processing Report

P.0. Box 72

Harrison, NY 10528

270. John Formica

Supermaterials

2020 Lakeside Avenue

Cleveland, $\mathrm{OH} 44114$

271. Jennifer M. Fox

Dyson Refractories Limited

R\&D Laboratory

Owler Bar

Sheffield S17 3BJ

UNITED KINGDOM

AIR MAIL

272. Edwin Frame

Southwest Research Institute

Division 2

P.0. Drawer 28510

Sari Antonio, TX 78284

273. Armanet Francois

French Scientific Mission

4101 Reservoir Road, N.W.

Washington DC 20007-2176

274. R. G. Frank

Techñolugy Assessment Group

10793 Bentley Pass Lane

Lovel and, $\mathrm{OH} 45140$ 
275. David J. Franus

Forecast International

22 Commerce Road

Newtown, CT 06470

276. Marc R. Freedman

NASA Lewis Research Center

21000 Brookpark Road

MS : 49-3

Cleveland, $\mathrm{OH} 44135$

277. Douglas Freitag

LTV Missiles Division

P.0. Box 650003

MS:WT-21

Dallas, TX 75265

278. Brian R.T. Frost

Argonne Nationa? Laboratory

9700 South Ca's Avenue

Building 9ne

Argonne, I! _.439

279. Lawrence R. Frost

Instron Corporation

100 Royal1 Street

Canton, MA 02021

280. George A. Fryburg

Norton/TRW Ceramics

7A-4 Raymond Avenue

Salem, NH 03079

281. Xiren $\mathrm{Fu}$

Shanghai Institute of Ceramics

Chinese Academy of Sciences

1295 Ding-xi Road

Shanghai 200050

CHINA AIR MAIL

282. John Gahimer

P.0. Box 1302

Dublin, $\mathrm{OH} 43017$

283. J. P. Gallagher

University of Dayton Research Institute

300 College Park, JPC-250

Dayton, $\mathrm{OH}$ 45469-0120
284. Tom Garritano

University of Tennessee

Science Alliance

101 South College

Knoxville, TN 37996-1328

285. Joy A. Garwood

Norton Company

Advanced Ceramics

Goddard Road

Northboro, MA 01532-1545

286. H. Maury Gatewood

Reynulds Metals Company Corporate R\&D

Fourth and Canal Streets

P.0. Box 27003

Richmond, VA 23261

287. L. J. Gauckler

ETH-Zurich

Sonneggstrasse 5

CH-8092 Zurich 8092

SWITZERLAND AIR MAIL

288. Peter A. Gaydos

Battelle Columbus Laboratories

505 King Avenue

Columbus, $\mathrm{OH} 43201$

289. George E. Gazza

U.S. Army Materials

Technology Laboratory

Ceramics Research Division

405 Arsenal Street

Watertown, MA 02172-0001

290. D. Gerster

CEA-DCOM

33 Rue De La Federation

Paris 75015

FRANCE

AIR MAIL

291. John Ghinazzi

Coors Technical Ceramics

Company

1100 Commerce Park Drive

Oak Ridge, TN 37830 
292. Robert Giddings

General Electric Company

Research Laboratory

P.0. Box 8

Schenectady, NY 12301

293. A. M. Glaeser

University of California

Materials Science and Mineral Engineering

Lawrence Berkeley Laboratory

Hearst Mining Building

Berkeley, CA 94720

294. Paul Glance

Concept Analysis

R\&D

950 Stephenson Highway

Dupont Automotive Development Building

Troy, MI 48007-7013

295. Joseph W. Glatz

Naval Air Propulsion Center

Systems Engineering Division

P.0. Box 7176, PE24

Trenton, NJ 08628

296. W. M. Goldberger

Superior Graphite Company

R\&D

2175 East Broad Street

Columbus, $\mathrm{OH} 43209$

297. Allan E. Goldman

U.S. Graphite, Inc.

907 West Outer Drive

Oak Ridge, TN 37830

298. Stephen T. Gonczy

Allied Signal Research

P.0. Box 5016

Des Plaines, IL 60017

299. Jeffrey M. Gonzales

GTE Products Corporation

Hawes Street

Towanda, PA 18848-0504

300. Robert J. Gottschall

U.S. Department of Energy

ER-131, MS:G-236

Washington, $D C 20545$
301. Earl Graham

Cleveland State University

Department of Chemical Engineering

Euclid Avenue at East 24th Street

Cleveland, $\mathrm{OH} 44115$

302. William A. Graham Lanxide Corporation P.0. Box 6077

Newark, DE 19714-6077

303. Robert E. Green, Jr. Johns Hopkins University Materials Science and Engineering Department Baltimore, MD 21218

304. Lance Groseclose

General Motors Corporation Allison Gas Turbine Division P.0. Box 420, MS:W-5 Indianapolis, IN 46206

305. Mark F. Gruninger Union Carbide Corporation Specialty Powder Business 1555 Main Street Indianapolis, IN 46224

306. Ernst Guge1

Cremer Forschungsinstitut GmbH\&CO.KG

Oeslauer Strasse 35

D-8633 Roedental 8633

GERMANY AIR MAIL

307. Donald L. Guile

Corning Glass Works

SP-DV-1-9

Corning, NY 14831

308. Bimleshwar P. Gupta

Solar Energy Research Institute

Mechanical and Industrial Technology Division

1617 Cole Boulevard Golden, CO 80401 
309. John P. Gyekenyesi

NASA Lewis Research Center 21000 Brookpark Road, MS:6-1

Cleveland, $\mathrm{OH} 44135$

310. Nabil S. Hakim

Detroit Diesel Corporation 13400 West Outer Drive Detroit, MI 48239

311. Philip J. Haley General Motors Corporation Vehicular Engineering P.0. Box 420, MS:T12A Indianapolis, IN 46236

312. Judith Hall

Fiber Materials, Inc. Biddeford Industrial Park 5 Morin Street Biddeford, ME 04005

313. Y. Harada

IIT Research Institute Nometallic Materials and Composites

10 West 35th Street

Chicago, IL 60616

314. R. A. Harmon

25 Schalren Drive

Latham, NY 12110

315. Amy Harmon-Barrett

Martin Marietta Laboratories 1450 South Rolling Road

Battimore, MD 21227

316. Norman H. Harris

Hughes Aircraft Company

P.0. Box 800520

Saugus, CA 91380-0520

317. Alan Hart

Dow Chemical Company

Advanced Ceramics Laboratory

17 i6 Building

Midl and, MI 48674
318. Pat E. Hart

Battelle Pacific Northwest Laboratories

Ceramics and Polymers

Development Section

P.0. Box 999

Richland, WA 99352

319. Stephen D. Hartline

Norton Company

Advanced Ceramics

Goddard Road

Northboro, MA 01532-1545

320. Michael H. Hasel korn

Caterpillar, Inc.

Engineering Research Materials

Technical Center, Building E

P.0. Box 1875

Peoria, IL 61656-1875

321. N. B. Havewala

Corning, Inc.

SP-PR-11

Corning, NY 14831

322. John Haygarth

Teledyne WAA Chang Albany

P. 0. Box 460

ATbany, OR 97321

323. Norman L. Hecht

University of Dayton Research Institute

300 College Park

Dayton, OH 45469-0172

324. Peter W. Heitman

General Motors Corporation

Allison Gas Turbine Division

P.0. Box 420, MS:W-5

Indianapolis, IN 46206-0420

325. Robert W. Hendricks

Virginia Polytechnic Institute and State University

Materials Engineering Department

210 Holden Hall

B1 acksburg, VA 24061-0237 
326. Wynne Henley

Hertel Cutting Technologies, Inc. 1000 Clearview Court

Oak Ridge, TN 37830

327. Thomas L. Henson

GTE Products Corporation

Chemical \& Metallurgical

Division

Hawes Street

Towanda, PA 18848

328. Thomas P. Herbell

NASA Lewis Research Center

21000 Brookpark Road

MS: 49-3

Cleveland, $\mathrm{OH} 44135$

329. Marlene Heroux

Rolls-Royce, Inc.

2849 Paces Ferry Road

Suite 450

At lanta, GA 30339-3769

330. Robert L. Hershey

Science Management Corporation

1255 New Hampshire Ave., N.W.

Suite 1033

Washington, DC 20036

331. Hendrik Heystek

Bureau of Mines

Tuscaloosa Research Center

P.0. Box L

University, AL 35486

332. Wallace C. Higgins

Norwal Unlimited

P.0. Box 1258

Alfred, NY 14802

333. Robert V. Hillery

GE Aircraft Engines

One Neumann Way, M.D. H85

Cincinnati, $\mathrm{OH} 45215$

334. Arthur Hindman

Instron Corporation

100 Royall Street

Canton, MA 02021
335. Jon Hines

American Ceramic Society, Inc.

757 Brooksedge Plaza Drive

Westerville, $\mathrm{OH}$ 43081-6136

336. Hans Erich Hintermann

CSEM

Materials and Micromechanics

Division

Rue Breguet 2

Neuchatel 2000

SWITZERLAND

AIR MAIL

337. Shinichi Hirano

Mazda R\&D of North

America, Inc.

1203 Woodridge Avenue

Ann Arbor, MI 48105

338. Tommy Hiraoka

NGK Locke, Inc.

1000 Town Center

Southfield, MI 48075

339. John M. Hobday

U.S. Department of Energy

Morgantown Energy Technology Center

Collins Ferry Road

P.0. Box 880

Morgantown, WV 26507

340. Greg Hoenes

Vista Chemical Company

900 Threadneedle

P.O. Box 19029

Houston, TX 77079-2990

341. Clarence Hoenig

Lawrence Livermore National Laboratory

P.0. Box 808, Mail Code L-369

Livermore, CA 94550

342. Thomas Hollstein

Fraunhofer-Institut fur Werkstoffmechanik IWM

Wohlerstrabe 11

D-7800 Freiburg

GERMANY AIR MAIL 
343. Richard Holt

National Research Council of Canada

Structures and Materials Laboratory Ottawa Ontario K1A OR6

CANADA AIR MAIL

344. Joseph Homeny University of Illinois

Department of Materials Science and Engineering

105 South Goodwin Avenue

Ceramics Building

Urbana, IL 61801

345. A. T. Hopper

Battelle Columbus

Laboratories

Metals and Ceramics Department

505 King Avenue

Columbus, OH 43201-2693

346. Michael Horgan

Materials Engineering Magazine

1100 Superior Avenue

Cleveland, $\mathrm{OH} 44114$

347. Woodie Howe

Coors Technical Ceramics Company

1100 Commerce Park Drive

Oak Ridge, TN 37830

348. Stephen M. Hsu

National Institute of

Standards and Technology

Gaithersburg, MD 20899

349. Hann S. Huang

Argonne National Laboratory

9700 South Cass Avenue

Argonne, IL 60439-4815

350. Gene Huber

Precision Ferrites \& Ceramics

5576 Corporate Drive

Cypress, CA 90630

351. M. L. Huckabee

GTE Laboratories, Inc.

40 Sylvan Road

Waltham, MA 02254
352. Harold A. Huckins

Princeton Advanced

Technology, Inc.

56 Finley Road

Princeton, NJ 08540

353. Fred R. Huettic

Advanced Magnetics, Inc.

45 Corey Lane

Mendham, NJ 07945

354. Bill Huffman

Zircar

110 North Main Street

Florida, NY 10921

355. Brian K. Humphrey

Lubrizol Petroleum

Chemicals Co.

3000 Town Center, Suite 1340

Southfield, MI 48075-1201

356. Robert M. Humrick

Dylon Ceramic Technologies

3100 Edgehill Road

Cleveland Heights, $\mathrm{OH} 44118$

357. Lorretta Inglehart

National Science Foundation

Division of Materials Research

1800 "G" Street, N.W.,

Room 408

Washington, DC 20550

358. Michael S. Inoue

Kyocera International, Inc.

KII Library

8611 Balboa Avenue

San Diego, CA 92123-1580

359. Osama Jadaan

University of Wisconsin-

Platteville

General Engineering Division

1 University Plaza

Platteville, WI 53818

360. Curtis A. Johnson

General Elertric Company

Corporate R\&D

Room MB-187

P.0. Box 8

Schenectady, NY 12301 
361. Sylvia Johnson

SRI International

333 Ravenswood Avenue

Menlo Park, CA 94025

362. Thomas A. Johnson Lanxide Corporation

1300 Marrows Road

P.0. Box 6077

Newark, DE 19714-6077

363. W. S. Johnson

Indiana University

One City Centre, Suite 200

Bloomington, IN 47405

364. Jill E. Jonkouski

U.S. Department of Energy

9800 South Cass Avenue

Argonne, IL 60439-4899

365. L. A. Joo

Great Lakes Research Corporation

P.0. Box 1031

Elizabethton, TN 37643

366. A. D. Joseph

SPX Corporation

700 Terrace Point

Muskegon, MI 49443

367. Adam Jostsons

Australian Nuclear Science \& Technology Organization

Lucas Heights Research Laboratories

New Ill awarra Road

Lucas Heights New South Wales

AUSTRALIA AIR MAIL

368. Matthew K. Juneau

Ethyl Corporation

451 Florida Street

Baton Rouge, LA 70801

369. Hartmut Kainer

Didier-Werke AG

Anlagentechnik Wiesbaden

Abraham-Lincoln-Str. 16

D-62 Wiesbaden

GERMANY AIR MAIL
370. Tom Kalamasz

Norton/TRW Ceramics

7A-4 Raymond Avenue

Salem, NH 03079

371. Lyle R. Kallenbach

Phillips Petroleum Company

R\&D

Mai1 Drop:123AL

Bartlesville, OK 74004

372. Nick Kamiya

Kyocera Industrial Ceramics

Corporation

2700 River Road

Des Plaines, IL 60018

373. Roy Kamo

Adiabatics, Inc.

3385 Commerce Park Drive

Columbus, IN 47201

374. S. Kang

GTE Laboratories, Inc.

40 Sylvan Road

Waltham, MA 02254

375. Chih-Chun Kao

Industrial Technology Research Institute

Materials Research Laboratories

195 Chung-Hsing Road, Sec. 4 Chutung Hsinchu 31015 R.O.C.

TAIWAN AIR MAIL

376. Keith R. Karasek

Allied-Signal, Inc.

Engineered Materials Research Center

50 East Algonquin Road

P.0. Box 5016

Des Plaines, IL 60017-5016

377. Robert E. Kasse1

Ceradyne, Inc.

3169 Redhill Avenue

Costa Mesa, CA 92626

378. Allan Katz

Wright Laboratory

Metals and Ceramics Division

WL/MLLM

Wright-Patterson AFB, OH 45433 
379. R. Nathan Katz

Worcester Polytechnic

Institute

Dept. of Mechanical

Engineering

100 Institute Road

Worcester, MA 01609

380. Ted Kawaguchi

Tokai Carbon America, Inc.

375 Park Avenue, Suite 3802

New York, NY 10152

381. Noritsugu Kawashima

TOSHIBA Corporation

Mechanical Engineering Laboratory

4-1 Ukishima-Cho

Kawasaki-Ku Kawasaki 210

JAPAN AIR MAIL

382. Lisa Kempfer

Penton Publishing

Materials Engineering

1100 Superior Avenue

Clevel and, $\mathrm{OH}$ 44114-2543

383. Frederick L. Kennard, III General Motors Corporation AC Rochester

Department 32-24, EB

1300 North Dort Highway

Flint, MI 48556

384. David 0. Kennedy

Lester B. Knight Cast Metals Inc.

549 West Randolph Street

Chicago, IL 60661

385. George Keros

Photon Physics

3175 Penobscot Building

Detroit, MI 48226

386. Pramod K. Khandelwal

General Motors Corporation

Allison Gas Turbine Division

P.0. Box 420, MS:W05

Indianapolis, IN 46206
387. Jim R. Kidwel1

Allied-Signal Aerospace Company

Garrett Auxiltary Power Division

P.0. Box 5227

Phoenix, AZ 85010

388. Han J. Kim

GTE Laboratories, Inc.

40 Sylvan Road

Waltham, MA 02254

389. Shin Kim

Korea Institute of Machinery \& Metals

66 Sangnam-dong, Changwon

Kyungnam 641-010

KOREA AIR MAIL

390. W. C. King

Mack Truck, Z-41

1999 Pennsylvania Avenue

Hagerstown, MD 21740

391. Carol Kirkpatrick

MSE, Inc.

CDIF Technical Library

P.0. Box 3767

Butte, MT 59702

392. Tony Kirn

Caterpillar, Inc.

Defense Products Department, JB7

Peoria, IL 61629

393. James D. Kiser

NASA Lewis Research Center

21000 Brookpark Road, MS:49-3

Clevel and, $\mathrm{OH} 44135$

394. Max Klein

Gas Research Institute

Thermodynamics

8600 West Bryn Mawr Avenue

Chicago, IL 60631

395. Richard N. Kleiner

Coors Ceramics Company

4545 McIntyre Street

Golden, CO 80403 
396. Stanley J. Klima NASA Lewis Research Center 21000 Brookpark Road MS : 6-1

Cleveland, $\mathrm{OH} 44135$

397. Chris E. Knapp Norton Advanced Ceramics of Canada Ltd. 8001 Daly Street Niagara Falls, Ontario L2G 6 S2 CANADA AIR MAIL

398. Albert S. Kobayashi University of Washington Mechanical Engineering Department MS : FU10

Seattle, WA 98195

399. Sinigeki Kobayashi

Toyota Central Research Labs, Inc.

Nagakute Aichi 480-11

JAPAN AIR MAIL

400. Richard A. Kole

Z-Tech Corporation

8 Dow Road

Bow, NH 03304

401. E. Kostiner

University of Connecticut

Chemistry Department, U-60

Storrs, CT 06269-3060

402. Kenneth A. Kovaly

Technical Insights, Inc.

P.0. Box 1304

Fort Lee, NJ 07024-9967

403. Ralph G. Kraft

Spraying Systems Company

North Avenue at Schmale Road

Wheaton, IL 60189-7900

404. Saunders B. Kramer

U.S. Department of Energy Advanced Propulsion Division CE-322, Forrestal Building Washington, DC 20585
405. Arthur Kranish

Trends Publishing, Inc.

1079 National Press Building

Washington, DC 20045

406. A. S. Krieger

Radiation Science, Inc.

P.0. Box 293

Belmont, MA 02178

407. Pieter Krijgsman

Ceramic Design International Holding B.V.

P.0. Box 68

Hattem 8050-AB

THE NETHERLANDS

AIR MAIL

408. Waltraud M. Kriven

University of Illinois

Materials Science and

Engineering Department

105 South Goodwin Avenue

Urbana, IL 61801

409. Edward J. Kubel, Jr.

ASM International Advanced Materials \& Processes

Materials Park, OH 44073

410. Dave Kupperman

Argonne National Laboratory

9700 South Cass Avenue

Argonne, IL 60439

411. Oh-Hun Kwon

Norton Company

Advanced Ceramics

Goddard Road

Northboro, MA 01532-1545

412. W. J. Lackey

Georgia Institute of Technology

Materials Science and Technology

Atlanta, GA 30332

413. Jai Lala

Tenmat Ltd.

40 Somers Road

Rugby Warwickshire CV22 7DH

ENGLAND AIR MAIL 
414. Hari S. Lamba

General Motors Corporation

Electro-Motive Division 9301 West 55th Street LaGrange, IL 60525

415. Richard L. Landingham Lawrence Livermore National Laboratory

Ceramics, Corrosion, and Thermochemistry

P.0. Box 808, L-369

Livermore, CA 94550

416. Charles J. Landry

Chand Kare Technical Ceramics

712 Flat Hill Road

Lumenburg, MA 01462

417. Manfred W. Langer

Volkswagen AG

Material Technology

3180 Wolfsburg 1

GERMANY AIR MAIL

418. James Lankford

Southwest Research Institute

Department of Materials Sciences

6220 Culebra Road

San Antonio, TX 78228-0510

419. Stanley B. Lasday

Business News Publishing Co. Manor Oak One

1910 Cochran Road, Suite 630

Pittsburgh, PA 15220

420. Mark S. Laser

Solar Turbines, Inc.

2211 Erie Street

San Diego, CA 92110

421. S. K. Lau

Carborundum ompany

Technology Division

P.0. Box 832, B- 100

Niagara Falls, NY 14302

422. Edward A. Lauder

Advanced Composite Materials Corporation

1525 South Buncombe Road

Greer, SC 29651-9208
423. J. Lawrence Lauderdale

Babcock \& Wilcox

Contract Research Division 1850 "K" Street, Suite 950

Washington, DC 20006

424. Harry A. Lawler

Carborundum Company

Technology Division

P.0. Box 832

Niagara Falls, NY 14302

425. Jean F. LeCostaouec

Textron Speciality Materials

2 Industrial Avenue

Lowe 11, MA 01851

426. Benson P. Lee

Interscience, Inc.

9718 Lake Shore Boulevard

Cleveland, $\mathrm{OH} 44108$

427. Burtrand I. Lee

Clemson University

Department of Ceramic Engineering

01 in $\mathrm{Hall}$

Clemson, SC 29634-0907

428. June-Gunn Lee

KIST

Structural Ceramic Lab

P.O. Box 131, Cheong-Ryang

SeouT 130-650

KOREA AIR MAIL

429. Ran-Rong Lee

Ceramics Process Systems

Corporation

155 Fortune Boulevard

Milford, MA 01757

430. Stan Levine

NASA Lewis Research Center

21000 Brookpark Road

MS : 49-3

Clevel and, $\mathrm{OH} 44135$

431. Alan V. Levy

Lawrence Berkeley Laboratory

One Cyclotron Road, MS:62-203

Berkeley, CA 94720 
432. Ai-Kang Li

Materials Research

Laboratories, ITRI

195-5 Chung-Hsing Road, Sec. 4

Chutung Hsinchu 31015 R.O.C.

TAIWAN AIR MAIL

433. Winston W. Liang

Hong Kong Industrial

Technology Centre Co. Ltd.

78 Tat Chee Avenue

4/F, HKPC Building

Howloon

HONG KONG AIR MAIL

434. Robert Licht

Norton Company

Advanced Ceramics

Goddard Road

Northboro, MA 01532-1545

435. E. Lilley

Norton Company

Advanced Ceramics

Goddard Road

Northboro, MA 01532-1545

436. Laura J. Lindberg

Allied-Signal Aerospace

Company

Garrett Fluid Systems Division

1300 West Warner

MS: 93-901-1207-4TT

P.0. Box 22200

Tempe, AZ 85284-2200

437. Leonard C. Lindgren

General Motors Corporation

Allison Gas Turbine Division

P.0. Box 420, Speed Code:T-20A

Indianapolis, IN 46206-0420

438. Hans A. Lindner

Cremer Forschungsinstitut GmbH\&.Co.KG

Oeslaver Strasse 35

D-8633 Rodental 8866

GERMANY AIR MAIL
439. Ronald E. Loehman

Sandia National Laboratories

Chemistry \& Ceramics

Department 1840

P.0. Box 5800

Albuquerque, NM 87185

440. Jeffrey C. Logas

Winona State University

Composite Materials Engineering

115 Pasteur Hall

Winona, MN 55987

441. Bill Long

Babcock \& Wilcox

P.0. Box 11165

Lynchburg, VA 24506

442. William D. Long

Wacker Chemicais (USA), Inc.

ESK Engineered Ceramics

50 Locust Avenue

New Canaan, CT 06840

443. L. A. Lott

EG\&G Idaho, Inc.

Idaho National Engineering Laboratory

P.0. Box 1625

Idaho Falls, ID 83415-2209

444. Raouf 0. Loutfy

MER Corporation

7960 South Kolb Road

Tucson, AZ 85706

445. Gordon R. Love

Aluminum Company of America

Alcoa Technical Center

Alcoa Center, PA 15069

446. Lydia Luckevich

Ortech International

2395 Speakman Drive

Mississauga Ontario L5K $1 B 3$

CANADA AIR MAIL 
447. James W. MacBeth

Carborundum Company

Structural Ceramics Division

P.0. Box 1054

Niagara Falls, NY 14302

448. H. MacLaren

General Electric Company

Thomson Laboratory, Materials

Engineering 36807

1000 Western Avenue

Lynn, MA 01910

449. George Maczura

Aluminum Company of America Industrial Chemicals Division 670 One Allegheny Square

Pittsburgh, PA 15212

450. David Maginnis

Tinker AFB

$O C-A L C / L I I R E$

Tinker AFB OK 73145-5989

451. Frank Maginnis

Aspen Research, Inc.

220 Industrial Boulevard

Moore, OK 73160

452. Tai-il Mah

Universal Energy Systems, Inc.

Ceramics and Composites

Research

4401 Dayton-Xenia Road

Dayton, OH 45432

453. Kenneth M. Maillar

Barbour Stocrivell Company

83 Linskey Way

Cambridge, MA 02142

454. Lorenzo Majno

Instron Corporation

100 Royall Street

Canton, MA 02021

455. S. G. Malghan

National Institute of Standards and Technology

I-270 \& Clopper Road

Gaithersburg, MD 20899
456. Lars Malmrup

United Turbine $A B$

Box 13027

Malmo S-200 44

SWEDEN AIR MAIL

457. John Mangels

Ceradyne, Inc.

3169 Redhill Avenue

Costa Mesa, CA 92626

458. Russel1 V. Mann

Matec Applied Sciences, Inc.

75 South Street

Hopkinton, MA 01748

459. William R. Manning

Champion Aviation Products Division

0ld Norris Road

P.0. Box 686

Liberty, SC 29657

460. Ken Marnoch

Amercom, Inc.

8928 Fullbright Avenue

Chatsworth, CA 91311

461. Robert A. Marra

Aluminum Company of America

Alcoa Technical Center

Advanced Ceramics Center - E

Alcoa Center, PA 15069

462. Chauncey L. Martin

3M Company

3M Center, Building 60-1N-01

St. Paul, MN 55144

463. Steven C. Mart in

Advanced Refractory

Technologies, Inc.

699 Hertel Avenue

Buffalo, NY 14207

464. Kelly J. Mather

Williams International

Corporation

2280 West Maple Road

P.0. Box 200

Walled Lake, MI 48088 
465. James $P$. Mathers

3M Company

3M Center

Building 201-3N-06

St. Pau1, MN 55144

466. Marshall Mayer

Instron Corporation

3815 Presidential Parkway, Suite 100

Atlanta, GA 30340

467. Ron Mayville

Arthur D. Little, Inc.

15-163 Acorn Park

Cambridge, MA 02140

468. F. N. Mazadarany

General Electric Company

Research Laboratory

Building K-1, Room MB-159

P.0. Box 8

Schenectady, NY 12301

469. James W. McCauley

Alfred University

NYS College of Ceramics

Binns-Merrill Hall

Alfred, NY 14802

470. Carolyn McCormick

Allied-Signal Aerospace Company

Garrett Auxiliary Power

Division

B1dg. 1303-206

P.0. Box 5227, MS: 9317-2

Phoenix, AZ 85010

471. Louis R. McCreight

2763 San Ramon Drive

Rancho Palos Verdes, CA 90274

472. Colin F. McDonald

McDonald Thermal Engineering

1730 Castelliana Road

La Jolla, CA 92037

473. B. J. McEntire

Norton Company, TRW Ceramics

Goddard Road

Northboro, MA 01532-1545
474. Chuck McFadden

Coors Ceramics Company

600 9th Street

Golden, CO 80401

475. Henry McFadden

Magnetic Bearings, Inc.

Engineering Library

609 Rock Road

Radford, VA 24141

476. Thomas D. McGee

Iowa State University

Materials Science and

Engineering Department

110 Engineering Annex

Ames, IA 50011

477. Carol McGill

Corning Inc.

Sullivan Park, FR-02-08

Corning, NY 14831

478. T. C. McLaren

Cameron Forged Products Company

P.0. Box 1212

Houston, TX 77251-1212

479. James McLaughlin

Sundstrand Power Systems

4400 Ruffin Road

P.0. Box 85757

San Diego, CA 92186-5757

480. Arthur F. McLean

6225 North Camino Almonte

Tucson, AZ 85718

481. Matt McMonigle

U.S. Department of Energy

Improved Energy Productivity Division

Forrestal Building, CE-231

Washington, DC 20585

482. Dennis McMurtry

EG\&G Idaho, Inc.

Idaho National Engineering Laboratory

P.0. Box 1625

Idaho Falls, ID 83415 
483. D. B. Meadowcroft

National Power Technology and Environmental Centre

Kelvin Avenue

Leatherhead Surrey KT22 7SE

ENGLAND AIR MAIL

484. Jo Meglen

11004 Birdfoot Court

Reston, VA 22091

485. Pankaj K. Mehrotra

Kennametal, Inc.

P.0. Box 639

Greensburg, PA 15601

486. Joseph J. Meindl

Reynolds International, Inc.

6003 West Broad Street

P.0. Box 27002

Richmond, VA 23261-7003

487. Michae1 D. Meiser

Allied-Signal Aerospace Company

Garrett Ceramic Components Division

19800 South Van Ness Avenue

Torrance, CA 90509

488. George Messenger

National Research Council of Canada

Engine Laboratory

Building M-7

Ottawa Ontario K1A OR6

CANADA AIR MAIL

489. D. Messier

U.S. Army Materials Technology Laboratory

SLCMT-EMC

405 Arsenal Street

Watertown, MA 02172-0001

490. Gary L. Messing

Pennsylvania State University

Ceramic Science and Engineering Department

119 Steidle Building

University Park, PA 16802
491. Arthur G. Metcalfe

Arthur G. Metcalfe and

Associates, Inc.

2108 East 24th Street

National City, CA 91950

492. R. Metselaar

Eindhoven University

Centre for Technical Ceramics

P.0. Box 513

Eindhoven $5600 \mathrm{MB}$

THE NETHERLANDS

AIR MAIL

493. Nancy S. Meyers

U.S. Department of Energy

Transportation Technologies

CE-30, Forrestal Building 6B-094

Washington, DC 20585

494. David J. Michael

Harbison-Walker Refractories Company

P.0. Box 98037

Pittsburgh, PA 1522.7

495. Ken Michaels

Chrysler Motors Corporation

Ceramics Development Metallurgical Processes

P.0. Box 1118, CIMS:418-17-09

Detroit, MI 48288

496. Bernd Michel

Institute of Mechanics

Fracture and Micromechanics Department

P.0. Box 408

D-9010 Chemnitz

GERMANY AIR MAIL

497. David E. Miles

Commission of the European Communities

rue de la Loi, 200

B-1049 Brussels

BELGIUM

AIR MAIL

498. John V. Milewski

Superkinetics, Inc.

P.0. Box 8029

Santa Fe, NM 87504 
499. Carl E. Miller

AC Rochester

1300 North Dort Highway, MS:32-31

Engineering Building B

Fl int, MI 48556

500. Mike Miller

MCGraw-Hill Aviation Week

Performance Materials

1156 15th Street, N.W.

Washington, DC 20005

501. Charles W. Miller, Jr.

Centorr Furnaces/Vacuum Industries

542 Amherst Street

Nashua, NH 03063

502. R. Mininni

Enichem America

2000 Cornwall Road

Monmouth Junction, NJ 08852

503. Michele V. Mitchell

Allied-Signal Aerospace Company

Garrett Ceramic Components Division

19800 South Van Ness Avenue

Torrance, CA 90501-1149

504. Howard Mizuhara

GTE - WESGO

477 Harbor Boulevard

Belmont, CA 94002

505. Helen Moeller

Babcock \& Wilcox

P.0. Box 11165

Lynchburg, VA 24506-1165

506. Francois R. Mollard

Metalworking Technology, Inc.

1450 Scalp Avenue

Johnstown, PA 15904

507. Phil Mooney

Panametrics

NDE Division

221 Crescent Street

Waltham, MA 02254
508. Thomas Morel

Ricardo North America

645 Blackhawk Drive

Westmont, IL 60559

509. Geoffrey P. Morris

3M Company

3M Traffic Control Materials

Division

B1dg. 209-BW-10, 3M Center

St. Pau1, MN 55144-1000

510. Jay A. Morrison

Rol1s-Royce, Inc.

Engineering and Information Center, Overlook 1

2849 Paces Ferry Road, Suite 450

Atl anta, GA 30339-3769

511. Joel P. Moskowitz

Ceradyne, Inc.

3169 Redhill Avenue

Costa Mesa, CA 92626

512. Brij Moudgil

University of Florida

Material Science and

Engineering

Gainesville, FL 32611

513. Christoph J. Mueller

Sprechsaal Publishing Group

P.0. Box 2962, Mauer 2

D-8630 Coburg

GERMANY AIR MAIL

514. Thomas W. Mullan

Vapor Technologies Inc.

345 Route 17 South

Upper Saddle River, NJ 07458

515. M. K. Murthy

MKM Consultants International

10 Avoca Avenue, Unit 1906

Toronto Ontario M4T 2B7

CANADA AIR MAIL

516. Solomon Musikant

TransCon Technologies, Inc.

Materials Science \& Engineering 1508 Waynesboro Road

Paoli, PA 19301 
517. David L. Mustoe

Custom Technical Ceramics 8041 West I 70 Service Road, Unit. 6

Arvada, CO 80002

518. Curtis V. Nakaishi

U.S. Department of Energy

Morgantown Energy Technology Center

Collins Ferry Road

P.0. Box 880

Morgantown, WV 26507-0880

519. Yoshio Nakamura

Faicera Research Institute 2-5-8 Hiyakunin-cho Shinjuku-Ko Tokyo

JAPAN AIR MAIL

520. K. S. Narasimhan Hoeganaes Corporation

River Road

Riverton, NJ 08077

521. Samuel Natansohn

GTE Laboratories, Inc.

40 Sylvan Road

Waltham, MA 02254

522. Robert Naum

Applied Resources, Inc.

P.0. Box 241

Pittsford, NY 14534

523. Malcolm Naylor

Cummins Engine Company, Inc.

P.0. Box 3005, Mail Code 50183

Columbus, IN 47202-3005

524. Jeffrey Neil

GTE Laboratories, Inc.

40 Sylvan Road

Wal tham, MA 02254

525. Fred A. Nichols

Argonne National Laboratory

9700 South Cass Avenue

MCT - Building 212

Argonne, IL 60439
526. H. Nicke1

Furschungszentrum Jüelich (KFA)

P.0. Box 1913 Jüelich 1-5170 BRD NRW

GERMANY AIR MAIL

527. Dale E. Niesz

Rutgers University

Center for Ceramic Research

P.0. Box 909

Piscataway, NJ 08855-0909

528. David M. Nissley

United Technologies Corporation

Pratt \& Whitney Aircraft

400 Main Street, MS:163-10

East Hartford, CT 06108

529. Richard D. Nixdorf

ReMaxCo Technologies, Inc.

11317 Snyder Road

Knoxville, TN 37932

530. Bernard North

Kennametal, Inc.

P.0. Box 639

Greensburg, PA 15601

531. Bruce E. Novich

Ceramics Process Systems

Corporation

155 Fortune Boulevard

Milford, MA 01757

532. Daniel Oblas

GTE Laboratories, Inc.

40 Sylvan Road

Wal tham, MA 02254

533. Don Ohanehi

Magnetic Bearings, Inc.

1908 Sussex Road

Blacksburg, VA 24060

534. Robert Orenstein

General Electric Company

55-112, River Road

Schenectady, NY 12345 
535. Norb Osborn

Aerodyne Dallas

151 Regal Row,

Suite 120

Dallas, TX 7524i

535. A. M. Paddick

BP International Limited

BP Research Centre, Main Library

Chertsey Road

Sunbury-on-Thames

Middlesex TW16 7LN

UNITED KINGDOM

AIR MAIL

537. Russell J. Page

Kanthal-Artcor

3001 Redhill Avenue, II-109

Costa Mesa, CA 92705

538. Richard Palicka

Cercom, Inc.

1960 Watson Way

Vista, CA 92083

539. Muktesh Paliwal

GTE Products Corporation

Hawes Street

Towanda, PA 18848

540. Joseph E. Palko

General Electric Company

55-113, River Road

Schenectady, NY 12345

541. Hayne Palmour, III

North Carolina State University

Materials Science and Engineering Dept.

Raleigh, NC 27605-7905

542. Joseph N. Panzarinu

Norton Company

Advanced Ceramics

Goddard Road

Northboro, MA 01532-1545

543. Pellegrino Papa

Corning Inc.

MP-WX-02-1

Corning, NY 14831
544. E. Beth Pardue

Technology for Energy

Corporation

One Energy Center

P.0. Box 22996

Knoxville, TN 37933-0996

545. Soon C. Park

3M Company

3M Center

Building 142-4N-02

P.0. Box 2963

St. Pau1, MN 55144

546. Hartmut Paschke

Schott Glaswerke

Christoph-Dorner-Strasse 29

D-8300 Landshut

GERMANY AIR MAIL

547. Marina R. Pascucci

GTE Laboratories, Inc.

40 Sylvan Road

Waltham, MA 02254

548. James W. Patten

Cummins Engine Company, Inc.

Materials Engineering

P.0. Box 3005, Mail Code 50183

Columbus, IN 47202-3005

549. Robert A. Penty

Eastman Kodak Company

KAD/D73 - 35612

901 Elmgrove Road

Rochester, NY 14653

550. Robert W. Pepper

Textron Specialty Materials

2 Industrial Avenue

Lowe11, MA 01851

551. Peter Perdue

Detroit Diesel Corporation

Research Advanced Development Group

13400 West Outer Drive, Speed Code A-07

Detroit, MI 48239-4001 
552. Bruce Peters

Dow Chemical Company

Building 52

Midl and, MI 48667

553. John J. Petrovic

Los Alamos National Laboratory

Group MST-4, MS:6771

Los Alamos, NM 87545

554. Frederick S. Pettit

University of Pittsburgh

Pittsburgh, PA 15261

555. Ben A. Phillips

Phillips Engineering Company

721 Pleasant Street

St. Joseph, MI 49085

556. Richard C. Phoenix

Ohmtek, Inc.

2160 Liberty Drive

Niagara Falls, NY 14302

557. Bruce J. Pletka

Michigan Technological University

Metallurgical and Materials Engineering Department

Houghton, MI 49931

558. John P. Pollinger

Garrett Ceramic Components

19800 Van Ness Avenue

Torrance, CA 90501

559. P. Popper

High Technology Ceramics International Journal

22 Pembroke Drive

West 1 ands Newcast le-under-Lyme Staffs ST5 2JN

ENGLAND AIR MAIL

560. F. Porz

Universitat Karlsruhe

Institut fur Keramik Im Maschinendau

Postfach 6980

D-7500 Karl sruhe

GERMANY

AIF MAIL
561. Harry L. Potma

Royal Netherlands Embassy

Science and Technology 4200 Linnean Avenue, N.W. Washington, DC 20008

562. Bob R. Powell

General Motors Research Laboratories

Metallurgy Department

30500 Mound Road

Box 9055

Warren, MI 48090-9055

563. Stephen C. Pred

ICD Group, Inc.

1100 Valley Brook Avenue

Lyndhurst, NJ 07071

564. Karl M. Prewo

United Technologies Research Center

411 Silver Lane

MS: 24

East Hartford, CT 06108

565. Peter E. Price

Industrial Materials Technology, Inc.

P.0. Box 9565

Andover, MA 01810

566. Joseph M. Proud

GTE Laboratories, Inc.

Materials Science Laboratory

40 Sylvan Road

Waltham, MA 02254

567. Vimal K. Pujari

Norton Company

Advanced Ceramics

Goddard Road

Northboro, MA 01532-1545

568. George Quinn

National Institute of

Standards and Technology

Ceramics Division, Bldg. 223

Gaithersburg, MD 20899 
569. Ramas V. Raman

Ceracon, Inc.

1101 North Market Boulevard, Suite 9

Sacramento, CA 95834

570. Charles F. Rapp

Owens Corning Fiberglass

2790 Columbus Road

Granville, OH 43023-1200

571. Dennis W. Readey

Colorado School of Mines

Department of Metallurgy and Materials Engineering

Golden, CO 80401

572. Wilfred J. Rebello

PAR Enterprises, Inc.

$12601 \mathrm{Clift}$ ( Hunt Lane

Clifton, VA 22024

573. Harold Rechter

Chicago Fire Brick Company

R\&D

7531 South Ashland Avenue

Chicago, IL 60620

574. Robert R. Reeber

U.S. Army Research office

P.0. Box 12211

Research Triangle Park, NC 27709

5.5. K. L. Reifsnider

Virginia Polytechnic Institute and State University

Department of Engineering Science and Mechanics

B1 acksburg, VA 24061

576. Paul E. Rempes

McDonnell Douglass Missle Systems Company

P.0. Box 516,

Mail Code: 1066086

St. Louis, MO 63166-0516

577. Gopal S. Revankar

John Deere Company

Metals Research

3300 River Drive

Moline, IL 61265
578. K. T. Rhee

Rutgers University

Mechanical Engineering

P.0. Box 909

Piscataway, NJ 08854

579. James Rhodes

Advanced Composite Materials

Corporation

1525 South Buncombe Road

Greer, SC 29651

580. Roy W. Rice

W. R. Grace and Company

7379 Route 32

Columbia, MD 21044

581. David W. Richerson

2093 East Delmont Drive

Salt Lake City, UT 84117

582. Tomas Richter

J. H. France Refractories

1944 Clarence Road

Snow Shoe, PA 16874

583. Michel Rigaud

Ecole Polytechnique

Campus Universite De Montreal

P.0. Box 6079, Station A

Montreal, P.Q Quebec H3C $3 A 7$

CANADA AIR MAIL

584. R. E. Riman

Rutgers University

Ceramics Engineering Department

P.0. Box 909

Piscataway, NJ 08855-0909

585. Barry Ringstrom

Superior Graphite Company

P.0. Box 2373

Smyrna, GA 30081

586. John E. Ritter

University of Massachusetts

Mechanical Engineering

Department

Amherst, MA 01003 
587. Frank L. Roberge

Allied-Signal Aerospace Company

Garrett Auxiliary Power Division

P.0. Box 5227

Phoenix, AZ 85010

588. W. Eric Roberts

Advanced Ceramic Technology, Inc. 990 " $F$ " Enterprise Street Orange, CA 92667

589. Martha Rohr U.S. Department of Energy DOE Oak Ridge Field Office Building 4500N P.0. Box 2008, MS:6269 Oak Ridge, TN 37831-6269

590. Y. G. Roman

TNO TPD Keramick

P.0. Box 595

Einhoven 5600 AN

HOLLAND AIR MAIL

591. Mark D. Roos

Carborundum Company

P.0. Box 156

Niagara Falls, NY 14302

592. Michael Rossetti

Arthur D. Little, Inc.

15 Acorn Park

Cambridge, MA 01240

593. Barry R. Rossing

Lanxide Corporation

1300 Marrows Road

Newark, DE 19714-6077

594. Steven L. Rotz

Lubrizol Corporation

29400 Lakel and Boulevard

Wickliffe, OH 44092

595. Bruce Rubinger

Global Competitiveness, Inc.

One Devonshire Place, Suite 1011

Boston, MA 02109
596. Robert Ruh

Wright Laboratory

WL/MLLM

Wright-Patterson AFB, OH 45433

597. Robert J. Russe11

17 Highgate Road

Framingham, MA 01701

598. L. William Sahley

Supermaterials Company

$24400 \mathrm{Highl}$ and Road

Richmond Heights, $\mathrm{OH} 44143$

599. Jon Salem

NASA Lewis Research Center 21000 Brookpark Center

Cleveland, $\mathrm{OH} 44135$

600. W. A. Sanders NASA Lewis Research Center 21000 Brookpark Road,

MS: 49-3

Cleveland, $\mathrm{OH} 44135$

601. J. Sankar

North Carolina A\&T State University

Department of Mechanical

Engineering

Greensboro, NC 27411

602. Yasushi Sato

NGK Spark Plugs (U.S.A.), Inc.

1200 Business Center Drive, Suite 300

Mt. Prospect, IL 60056

603. Maxine L. Savitz

Allied-Signal Aerospace Company

Garrett Ceramic Components Division

19800 South Van Ness Avenue

Torrance, CA 90501

604. Ashok Saxena

Georgia Institute of Technology

Materials Engineering

Atlanta, GA 30332-0245 
605. David W. Scanlon Instron Corporation 100 Royall Street Canton, MA 02021

606. Charles A. Schacht

Schacht Consulting Services 12 Holl and Road Pittsburgh, PA 15235

607. James Schienle

Allied-Signal Aerospace Company

Garrett Auxiliary Power

Division

2739 East Washington Street

P.0. Box 5227, MS:1302-2P

Phoenix, AZ 85010

608. John C. Schneider

San Juan Technologies

P.0. Box 49326

Colorado Springs, CO 80949-9326

609. Gary Schnittgrund

Rocketdyne, BA05

6633 Canoga Avenue

Canoga Park, CA 91303

610. Mark Schomp

Lonza, Inc.

Corporate Headquarters

17-17 Route 208

Fair Lann, NJ 07410

611. Joop Schoonman

Delft University of Technology

Laboratory for Inorganic Chemistry

P.0. Box 5045

2600 GA Delft

THE NETHERLANDS

AIR MAIL

612. John Schuldies

Industrial Ceramic

Technology, Inc.

37 Enterprise Drive

Ann Arbor, MI 48103
613. Robert B. Schulz

U.S. Department of Energy

Office of Transportation

Materials

CE-34, Forrestal Building

Washington, DC 20585

614. Murray A. Schwartz

Materials Technology Consulting, Inc.

30 Orchard Way, North

Potomac, MD 20854

615. Peter Schwarzkopf

SRI International

333 Ravenswood Avenue

Menlo Park, CA 94025

616. Thomas C. Schweizer

Princeton Economic

Research, Inc. 12300 Twinbrook Pkwy., Suite 650

Rockville, MD 20852

617. William T. Schwessinger

Multi-Arc Scientific

Coatings

1064 Chicago Road

Troy, MI 48083-4297

618. W. D. Scott

University of Washington

Materials Science Department

Mail Stop:FB10

Seattle, WA 98195

619. Nancy Scoville

Thermo Electron Technologies

74 West Street

P.0. Box 9046

Waltham, MA 02254-9046

620. Brian Seegmiller

Coors Ceramics Company

600 9th Street

Golden, CO 80401 
621. T. B. Selover

AICRE/DIPPR

3575 Traver Road

Shaker Heights, OH 44122

622. J. H. Selverian

GTE Laboratories, Inc.

40 Sylvan Road

Wal tham, MA 02254

623. Charles E. Semler

Semler Materials Services

4160 Mumford Court

Columbus, $\mathrm{OH} 43220$

624. Thomas Service

Service Engineering Laboratory

324 Wells Street

Greenfield, MA 01301

625. Kish Seth

Ethyl Corporation

R\&D Laboratories

P.0. Box 341

Baton Rouge, LA 70821

626. Karleen Seybold

Allied-Signal Aerospace Company

Garrett Auxiliary Power

Division

P.0. Box 5227

Phoenix, AZ 85010

627. William J. Shack

Argonne National Laboratory

9700 South Cass Avenue

Building 212

Argonne, IL 60439

628. Peter T. B. Shaffer

Technical Ceramics Laboratories, Inc. 4045 Nine/McFarland Drive

Alpharetta, GA 30201

629. Richard K. Shaltens

NASA Lewis Research Center

21000 Brookpark Road,

MS: $301-2$

Cleveland, OH 44135
630. Robert S. Shane

238 Hemlock Road

Wynnewood, PA 19096

631. Daniel Shanefield

Rutgers University

Ceramics Engineering Department

P.0. Box 909

Piscataway, NJ 08855-0909

632. Ravi Shankar

Chromalloy

Research and Technology Division

Blaisdell Road

Orangeburg, NY 10962

633. Terence Sheehan

Alpex Wheel Company

727 Berkley Street

New Milford, NJ 07646

634. Dinesh K. Shetty

University of Utah

304 EMRO, Dept. of Materials

Science and Engineering

Salt Lake City, UT 84112

635. Masahide Shimizu

Ceramic Society of Japan

2-2-503 Takiyama 6-chome

Higashikurume-Shi Tokyo 203

JAPAN AIR MAIL

636. John Shipinski

Toyota Technical Center

U.S.A., Inc.

Technical Research Department

2000 Town Center, Suite 500

Southfield, MI 48075

637. Thomas Shreves

American Ceramic Society, Inc.

Library

757 Brooksedge Plaza Drive

Westerville, $\mathrm{OH}$ 43081-2821

638. Jack D. Sibold

Coors Ceramics Company

Contracts for Corporate

Technology

4545 McIntyre Street

Golden, CO 80403 
639. George H. Siegel

Point North Associates, Inc.

P.0. Box 907

Madison, NJ 07940

640. Richard Silberglitt

Technology Assessment and Transfer, Inc.

133 Defense Highway, \#212

Annapol is, MD 21401

641. Mary Silverberg

Norton Company

Advanced Ceramics Library

Goddard Road

Northboro, MA 01532-1545

642. Gurpreet Singh

Department of the Navy

Internal Combustion \& Gas

Turbine Engine Division Code 56×31

Washington, DC 20362-5101

643. Maurice J. Sinnott

University of Michigan

Chemical and Metallurgical

Engineering

5106 IST Building

Ann Arbor, MI 48109-2099

644. John Skildum

3M Company

3M Center

Building 224-2S-25

St. Paul, MN 55144

645. David P. Smith

Hoskins, Rees \& Smith

1910 Cochran Road

Manor Oak II, Suite 658

Pittsburgh, PA 15220

646. Richard H. Smoak

Smoak \& Associates

3554 Hollyslope Road

Altadena, CA 91001-3923
647. Jay R. Smyth

Allied-Signal Aerospace Company

Garrett Auxiliary Power

Division

2739 East Washington Street

P.0. Box 5227

MS: $93-173 / 1303-207$

Phoenix, AZ 85010

648. Edward A. Snajdr

Premier Refractories

and Chemicals, Inc.

P.0. Box 392

Findlay, $\mathrm{OH} 44815$

649. Rafal A. Sobotowski

British Petroleum Company

Technical Center, Broadway

3092 Broadway Avenue

Cleveland, $\mathrm{OH} 44115$

650. A. G. Solomah

SAC International Ltd.

1445 Bonhill Road, \# 13

Mississauga Ontario L5T 1 V3

CANADA AIR MAIL

651. S. Somiya

Nishi Tokyo University

3-7-19 Seijo, Setagaya

Tokyo 157

JAPAN AIR MAIL

652. Boyd W. Sorenson

DuPont Lanxide Composites

1300 Marrows Road

P.0. Box 6077

Neward, DE 19707

653. Charles A. Sorrell

U.S. Department of Energy

Advanced Industrial

Concepts Division

CE-232, Forrestal

Building

Washington, DC 20585 
654. C. Spencer

EA Technology

Capenhurst Chester CH1 6ES

UNITED KINGDOM AIR MAIL

655. Allen Spizzo

Hercules, Inc.

Hercules Plaza

Wilmington, DE 19894

656. Richard M. Spriggs

Alfred University

Center for Advanced Ceramic Technology

Alfred, NY 14802

657. Charles Spuckler

NASA Lewis Research Center

21000 Brookpark Road

MS: $5-11$

Cleveland, $\mathrm{OH}$ 44135-3191

658. M. Srinivasan

Material Solutions

P.0. Box 663

Grand Island, NY 14702-0663

659. Gordon L. Starr

Cummins Engine Company, Inc.

Design \& Technology

P.0. Box 3005

Mail Code 50182

Columbus, IN 47202-3005

660. Jim Stevenson

Windrock, Incorporated

154 Fairbanks Plaza

Oak Ridge, TN 37830

661. Tom Stillwagon

Allied-Signal Aerospace Company

Garrett Ceramic Components Division

19800 South Van Ness Avenue

Torrance, CA 90501

662. Harold L. Stocker

General Motors Corporation

Allison Gas Turbine Division P.0. Box 420

Indianapolis, IN 46206
663. Paul D. Stone

Dow Chemical USA

1776 "Eye" Street, N.W., Suite 575

Washington, DC 20006

664. Roger S. Storm

Carborundum Company

Technology Division

P.0. Box 337

Niagara Falls, NY 14302-0337

665. Peter A. Stranges

4 Chittenden Lane

Owings Mills, MD 21117

666. F. W. Stringer

Aero \& Industrial Technology Ltd.

P.0. Box 46, Wood Top

Burnley Lancashire BB11 4BX

UNITED KINGDOM AIR MAIL

667. Thomas N. Strom

NASA Lewis Research Center

21000 Brookpark Road

MS:86-6

Cleveland, $\mathrm{OH} 44135$

668. M. F. Stroosnijder

Institute for Advanced Materials

Joint Research Centre

21020 Ispra (VA)

ITALY AIR MAIL

669. Karsten Styhr

30604 Ganado Drive

Rancho Palos Verdes, CA 90274

670. T. S. Sudarshan

Materials Modification, Inc.

2929-PI Eskridge Center

Fairfax, VA 22031

671. M. J. Sundaresan

University of Miami

Mechanical Engineering Department

P.0. Box 248294

Coral Gables, FL 33124 
672. Patrick L. Sutton

U.S. Department of Energy Advanced Propulsion Division CE-322, Forrestal Building Washington, DC 20585

673. Willard H. Sutton United Technologies Corporation

Research Center, MS:24

Silver Lane

East Hartford, CT 06108

674. Ron Sviben

100 Indel Avenue

Rancocas, NJ 08073

675. J. J. Swab

U.S. Army Materials Technology Laboratory

Ceramics Research Division

SLCHT-EMC

405 Arsenal Street

Watertown, MA 02172

676. Robert E. Swanson

Metalworking Technology, inc.

1450 Scalp Avenue

Johnstown, PA 15904

677. Scott L. Swartz

Battelle Columbus Laboratories

Metals and Ceramics

505 King Avenue

Columbus, $\mathrm{OH} 43201$

678. Steve Szaruga

Air Force Wright Aeronautical Laboratory

Materials Directorate

WL/MLBC

Wright-Patterson

AFB, OH 45433-6533

679. Yo Tajima

NGK Spark Plug Company, Ltd.

NTK Technical Ceramic Division 2808 Iwasaki

Komaki-shi Aichi-ken 485

JAPAN AIR MAIL
680. Fred Teeter

5 Tralee Terrace

East Amherst, NY 14051

681. Monika 0. Ten Eyck

Carborundum Company

Technology Division

P.0. Box 832

Niagara Falls, NY 14302

682. David F. Thompson

Corning Glass Works

SP-DV-02-1

Corning, NY 14831

683. Merle L. Thorpe

Hobart Tafa Technologies, Inc.

146 Pembroke Road

Concord, NH 03301

684. Eberhard Tiefenbacher

Daimler-Benz AG Abt. FIS

Mercedes-Strabe 136

Stuttgart 60

GERMANY AIR MAIL

685. T. Y. Tien

University of Michigan

Materials Science and

Engineering Department

Dow Building

Ann Arbor, MI 48103

686. Julian M. Tishkoff

Air Force Office of Scientific Research

AFOSR/NA

Bolling AFB, DC 20332-6448

687. D. M. Tracey

Norton Company

Advanced Ceramics

Goddard Road

Northboro, MA 01532-1545

688. Dick Trippett

General Motors Corporation

Allison Gas Turbine Division

P. O. Box 420, MS:W-16

Indianapol is, IN 46206-0420 
689. L. J. Trostel, Jr.

Box 199

Princeton, MA 01541

690. W. T. Tucker

General Electric Company

Corporate R\&D

Building K1-4C35

P.0. Box 8

Schenectady, NY 12301

691. Masanori Ueki

Nippon Steel Corporation

Central R\&D Bureau

1618 Ida

Nakahara-Ku Kawasaki 211

JAPAN AIR MAIL

692. Filippo M. Ugolini

ATA Studio

Via Degli Scipioni, 268A

ROMA 00192

ITALY AIR MAIL

693. Donald L. Vaccari

Allison Gas Turbines

P.0. Box 420

Speed Code 549

Indianapolis, IN 46206-0420

694. Carl F. Van Conant

Boride Products, Inc.

2879 Aero Park Drive

Traverse City, MI 49684

695. Marcel H. Van De Voorde

Commission of the European Communities

Institute for Advanced

Materials

Joint Research Centre

P.0. Box 2

1755 ZG Petten

THE NETHERLANDS AIR MAIL

696. 0. Van Der Biest

Katholieke Universiteit Leuven

Departement Metaalkunde en Toegepaste

de Croylaan 2

B-3030 Leuven

BELGIUM AIR MAIL
697. Michael Vannier

Washington University, St. Louis

Mallinckrodt Institute of Radiology

510 South Kings Highway

st. Louis, MO 63110

698. Stan Venkatesan

Southern Coke \& Coal

Corporation

P.0. Box 52383

Knoxville, TN 37950

699. V. Venkateswaran

Carborundum Company

Niagara Falls R\&D Center

P.0. Box 832

Niagara Falls, NY 14302

700. Dennis Viechnicki

U.S. Army Materials Technology Laboratory

405 Arsenal Street

Watertown, MA 02172-0001

701. Ted Vojnovich

U.S. Department of Energy

Office of Energy Research, ER-42

Washington, DC 20585

702. John D. Volt

E. I. Dupont de Nemours \& Company, Inc.

P.0. Box 80262

Wilmington, DE 19880

703. John B. Wachtman

Rutgers University

Ceramics Department

P.0. Box 909

Piscataway, NJ 08855

704. Shigetaka Wada

Toyota Central Research Labs, Inc.

Nagakute Aichi 480-11

JAPAN AIR MAIL 
705. Janet Wade

Allied-Signal Aerospace Company

Garrett Auxiliary Power

Division, Department 93-772

P.0. Box 5227, MS:1303-2

Phoenix, AZ 85010

706. Richard L. Wagner

Ceramic Technologies, Inc.

537 Turtle Creek South Drive, Suite 240

Indianapolis, IN 46227

707. J. Bruce Wagner, Jr.

Arizona State University

Center for Solid State Science

Tempe, AZ 85287-1704

708. Daniel J. Wahlen

Kohler, Co.

$444 \mathrm{Highl}$ and Drive

Kohler, WI 53044

709. Ingrid Wahlgren

Royal Institute of Technology

Studsvik Library

S-611 82 Nykoping

SWEDEN AIR MAIL

710. Ron H. Walecki

Allied-Signal Aerospace

Company

Garrett Ceramic Components

Division

19800 South Van Ness Avenue

Torrance, CA 90501

711. Michael S. Walsh

Vapor Technologies Inc.

2100 Central Avenue

Boulder, CO 80301

712. Chien-Min Wang

Industrial Technology Research Institute

Materials Research Laboratories

195 Chung-Hsing Road, Sec. 4

Chutung Hsinchu 31015 R.O.C.

TAIWAN

AIR MAIL
713. Robert M. Washburn

ASMT

11203 Colima Road

Whittier, CA 90604

714. Gerald Q. Weaver

Carborundum Specialty Products

42 Linus Allain Avenue

Gardner, MA 01440-2478

715. Karen E. Weber

Detroit Diesel Corporation

Technology and Planning

13400 West Outer Drive

Detroit, MI 48239-4001

716. R. W. Weeks

Argonne National Laboratory

MCT-212

9700 South Cass Avenue

Argonne, IL 60439

717. Ludwig Weiler

ASEA Brown Boveri AG

Corporate Research

Eppelheimer Str. 82

D-6900 Heidelberg

GERMANY AIR MAIL

718. James Wessel

Dow Corning Corporation

1800 "M" Street, N.W.,

Suite 325 South

Washington, DC 20036

719. Robert D. West

Therm Advanced Ceramics

P.0. Box 220

Ithaca, NY 14851

720. Thomas J. Whalen

Ford Motor Company

26362 Harriet

Dearborn Heights, MI 48127

721. Ian A. White

Hoeganaes Corporation

River Road

Riverton, NJ 08077 
722. Alan Whitehead General Electric Company

1 River Road, 55-111

Schenectady, NY 12345

723. Sheldon M. Wiederhorn National Institute of Standards and Technology Building 223, Room A329 Gaithersburg, MD 20899

724. John F. Wight Alfred University McMahon Building Alfred, NY 14802

725. D. S. Wilkinson McMaster University Materials Science and Engineering Department 1280 Main Street, West Hamilton Ontario L8S 4L7 CANADA AIR MAIL

726. James C. Williams General Electric Company Engineering Materials Technology Labs

One Neumann Way

Mail Drop: H85

Cincinnati, $\mathrm{OH}$ 45215-6301

727. Janette R. Williams Kollmorgen Corporation PCK Technology Division 150 Motor Parkway, \#262 Hauppauge, NY 11788-5108

728. Steve J. Williams RCG Hagler Bailly, Inc. 1530 Wilson Boulevard, Suite 900

Arlington, VA 22209-2406

729. Craig A. Will kens

Norton Company

Advanced Ceramics

Goddard Road

Northboro, MA 01532-1545
730. Roger R. Wills TRW, Inc.

Valve Division 1455 East 185th Street Cleveland, $\mathrm{OH} 44110$

731. David Gordon Wilson

Massachusetts Institute of Technology

Mechanical Eng ineering Department

77 Massachusetts Avenue, Room 3-455

Cambridge, MA 02139

732. Matthew F. Winkler

Seaworthy Systems, Inc.

P.0. Box 965

Essex, CT 06426

733. Gerhard Winter

Hermann C. Starck. Berl in GmbH, Werk Goslar

P.0. Box 2540

D-3380 Gos lar 3380

GERMANY AIR MAIL

734. W. L. Winterbottom

Ford Motor Company

Material Systems Reliability Department

20000 Rotunda Drive

SRL, Room E-3182

P.0. Box 2053

Dearborn, MI 48121

735. David G. Wirth

Coors Ceramics Company

600 9th Street

Golden, CO 80401

736. Thomas J. Wissing

Eaton Corporation

Engineering \& Research Center P.0. Box 766

Southfield, MI 48037

737. James C. Withers

MER Corporation

7960 South Kolb Road

Building $F$

Tucson, AZ 85706 
738. Da7e E. Wittmer

Southern IIlinois University

Mechanical Engineering Department

Carbondale, IL 62901

739. Warren W. Wolf

Owens Corning Fiberglass

2790 Columbus Road, Route 16

Granville, OH 43023

740. George W. Wolter

Howmet Turbine Components

Corporation

Technical Center

699 Benston Road

Whitehall, MI 49461

741. James C. Wood

NASA Lewis Research Center

21000 Brookpark Road

MS: 86- 6

Cleveland, $\mathrm{OH} 44135$

742. Marrill Wood

LECO Corporation

Augusta Division

P.0. Box 211688

Augusta, GA 30917-1688

743. Wayne L. Worrell

University of Pennsylvania

Department of Materials

Science and Engineering

3231 Walnut Street

Philadelphia, PA 19104

744. John F. Wosinski

Corning Inc.

ME-2 E-5 H8

Corning, NY 14830

745. Ian G. Wright

Battelle Columbus Laboratories

505 King Avanue

Columbus, $\mathrm{OH} 43201$

746. Ruth Wroe

ERDC

Metals \& Materials Industries

Division

Capenhurst Chester CH1 6ES

ENGLAND AIR MAIL
747. Bernard J. Wrona

Advanced Composite Materials

Corporation

1525 South Buncombe Road

Greer, SC 29651

748. Carl C. M. Wu

Naval Research Laboratory

Ceramic Branch, Code 6373

Washington, DC 20375

749. John C. Wurst

University of Dayton Research Institute

300 College Park

Dayton, OH 45469-0101

750. Neil Wyant

ARCH Development Corp.

9700 South Cass Avenue

Building 202

Argonne, IL 60439

751. Roy Yamamoto

Texaco Inc.

P.0. Box 509

Beacon, NY 12508-0509

752. John Yamanis

All ied-Signal, Inc.

Ceramics Program

P.0. Box 1021

Morristown, NJ 07962-1021

753. Harry C. Yeh

Allied-Signal Aerospace

Company

Garrett Ceramic Components

Division

19800 South Van Ness Avenue

Torrance, CA 90509

754. Hiroshi Yokoyama

Hitachi Research Lab

4026 Kuji-Cho

Hitachi-shi Ibaraki 319-12

JAPAN AIR MAIL

755. Thomas M. Yonushonis

Cummins Engine Company, Inc.

P. 0. Box 3005

Mail Code 50183

Columbus, IN 47202-3005 
756. Thomas J. Yost

Corning, Inc.

Technical Products Division,

Main P1 ant 21-1-2

Corning, NY 14831

757. Jong Yung

Sundstrand Aviation Operations

Department 789-6

4747 Harrison Avenue

Rockford, IL 61125

758. A. L. Zadoks

Caterpillar Inc.

Technical Center, Building L

P.0. Box 1875

Peoria, IL 61656-1875

759. Avi Zangvil

University of Illinois

Materials Research Laboratory

104 South Goodwin Avenue

Urbana, IL 61801

760. Charles H. Zenuk

Transtech

6529 East Calle Cavalier

Tucson, AZ 85715

761. Anne Marie Zerega

U.S. Department of Energy

Office of Energy Research, ER-42

Washington, DC 20585

762. Ken Zillmer

Applied Test Systems, Inc.

2571 Mt. Gallant Road

Rock Hill, SC 29730

763. Carl Zweben

General Electric Company

P.0. Box 8555, VFSC/V4019

Philadelphia, PA 19101

764. Klaus M. Zwilsky

National Research Council

National Materials

Advisory Board

2101 Constitution Avenue

Washington, DC 20418
765. Department of Energy

DOE Field Office, Oak Ridge

Assistant Manager for Energy

Research and Development

P.0. Box 2001

Oak Ridge, TN 37831-8501

766-775. Department of Energy

Office of Scientific and

Tectoical Information

Office of Information

Services

P.0. Box 62

Oak Ridge, TN 37831

For distribution by microfiche as shown in DOE/OSTI-4500, Distribution Category UC-332 (Ceramics/Advanced Materials). 

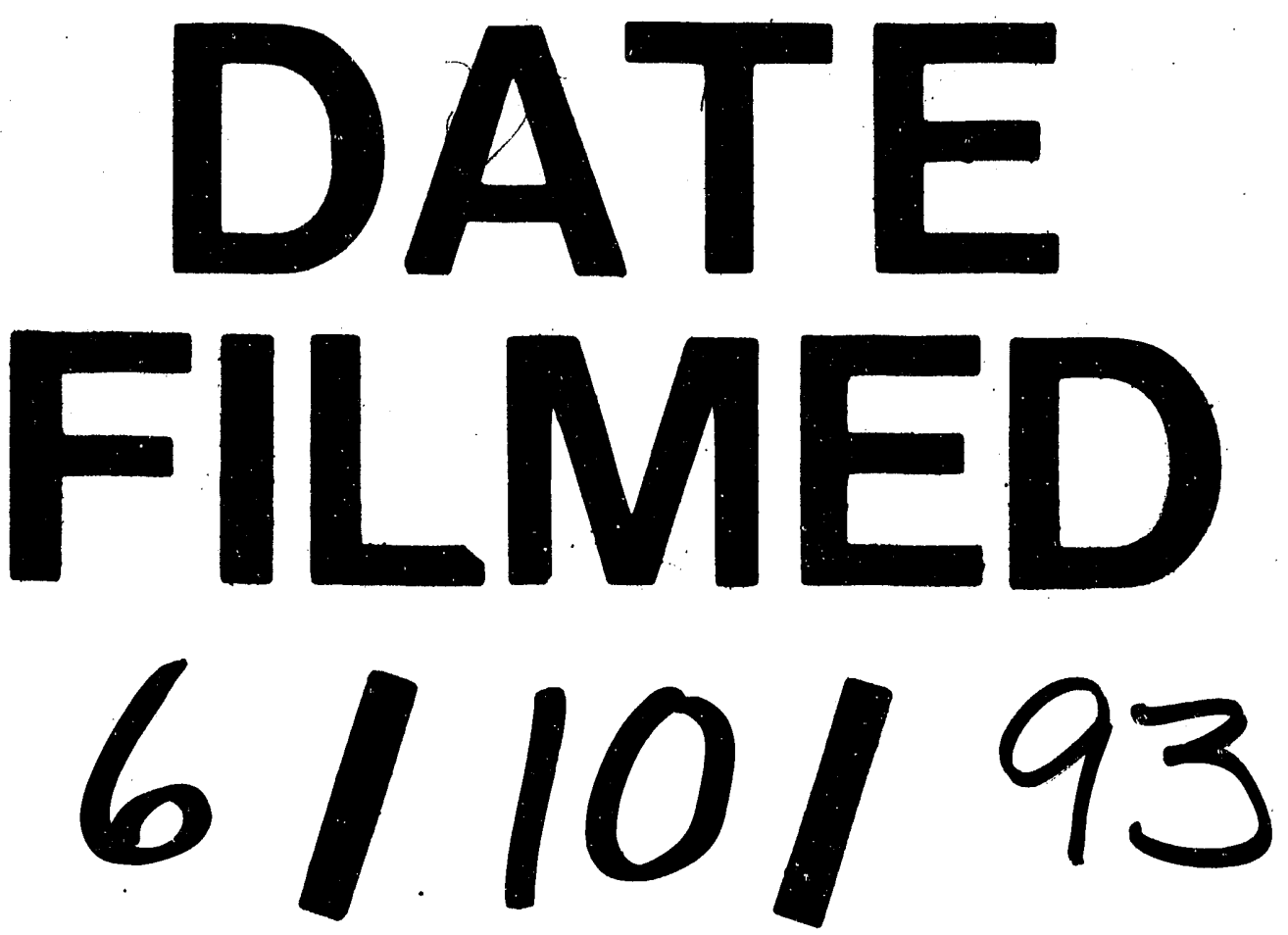
